\title{
Coulomb Scattering in the Massless Nelson Model I.
}

\section{Foundations of Two-Electron Scattering}

\author{
Journal Article \\ Author(s): \\ Dybalski, Wojciech; Pizzo, Alessandro \\ Publication date: \\ 2014-01 \\ Permanent link: \\ https://doi.org/10.3929/ethz-b-000081037
}

Rights / license:

In Copyright - Non-Commercial Use Permitted

Originally published in:

Journal of Statistical Physics 154(1-2), https://doi.org/10.1007/s10955-013-0857-y 


\title{
Coulomb Scattering in the Massless Nelson Model I. Foundations of Two-Electron Scattering
}

\author{
W. Dybalski • A. Pizzo
}

Received: 29 May 2013 / Accepted: 26 September 2013 / Published online: 26 October 2013

(C) Springer Science+Business Media New York 2013

\begin{abstract}
We construct two-electron scattering states and verify their tensor product structure in the infrared-regular massless Nelson model. The proof follows the lines of HaagRuelle scattering theory: Scattering state approximants are defined with the help of two time-dependent renormalized creation operators of the electrons acting on the vacuum. They depend on the ground state wave functions of the (single-electron) fiber Hamiltonians with infrared cut-off. The convergence of these approximants as $t \rightarrow \infty$ is shown with the help of Cook's method combined with a non-stationary phase argument. The removal of the infrared cut-off in the limit $t \rightarrow \infty$ requires sharp estimates on the derivatives of these ground state wave functions w.r.t. electron and photon momenta, with mild dependence on the infrared cut-off. These key estimates, which carry information about the localization of the electrons in space, are obtained in a companion paper with the help of iterative analytic perturbation theory. Our results hold in the weak coupling regime.
\end{abstract}

\section{Introduction and Results}

The last two decades have witnessed substantial progress in the mathematical understanding of scattering of light and matter in the framework of non-relativistic QED. These advances provided a rigorous foundation for the physical description of the Compton [5, 6, 20, 24, 25] and Rayleigh scattering [7-10, 13, 18, 19, 22, 28, 29], involving one massive particle (the 'electron') and many massless excitations ('photons'). However, the case of Coulomb scattering, i.e., collisions of two electrons in the presence of photons, has remained outside of

Dedicated to Herbert Spohn on the occasion of his 65th birthday.

With gratitude for all he taught us about the dynamics of matter and radiation.

W. Dybalski ( $\square)$

ETH Zurich, Zurich, Switzerland

e-mail: dybalski@ma.tum.de

A. Pizzo

UC Davis, Davis, CA, USA

e-mail:pizzo@math.ucdavis.edu 
the scope of these investigations. This is a serious gap in our understanding of physics, given the tremendous importance of this scattering process, ranging from Rutherford's discovery of the structure of atoms to modern high-energy physics experiments. This paper is a first part of a larger investigation, the goal of which is to put this important process on rigorous grounds.

A general framework of scattering theory for several electrons in models of nonrelativistic QED, which has its roots in Haag-Ruelle scattering theory [21, 27], was known to experts already more than three decades ago $[2,3,14]$. However, a complete construction of scattering states was only possible in the presence of a fixed infrared cut-off (or non-zero photon mass), due to infrared- and infraparticle problems. Depending on the structure of the interaction, one can distinguish the infrared-regular case, in which the physical Hilbert space of the theory contains single-electron states, and the infrared-singular case in which the electron is inevitably accompanied by soft photons (i.e., it is an infraparticle). For processes involving one electron and photons, scattering states have been constructed in both cases with the help of a sophisticated multiscale technique [24, 25]. However, scattering of several electrons has remained outside of the scope of previous investigations, both in the infrared-regular and infrared-singular situation. In this paper we provide a construction of two-electron scattering states in the massless, infrared-regular Nelson model without an infrared cut-off. (We do not include a two-body quantum mechanical potential between the electrons, but only the interaction mediated by photons.) Although the infraparticle problem does not arise in this case, the infrared structure of the model is non-trivial and requires major refinements of the multiscale technique. To clarify the origin of these new difficulties and describe our methods to tackle them, we explain below in non-technical terms the main steps of our analysis. We are confident that similar steps will also appear in a future construction of scattering states of several infraparticles, where they should be combined with an analysis of soft photons. However, due to the absence of definite results, we refrain from extensive speculations on the infrared-singular situation here. Brief remarks on this case are given below equation (1.12).

We consider the translationally invariant, massless Nelson model. It describes secondquantized non-relativistic particles, which we call electrons (though they obey the Bose statistics), and massless scalar bosons, which we call photons. The Hamiltonian of this model is a self-adjoint operator on the physical Hilbert space $\mathcal{H}$ which is the tensor product of the electron Fock space $\Gamma\left(\mathfrak{h}_{\mathrm{e}}\right)$ and the photon Fock space $\Gamma\left(\mathfrak{h}_{\mathrm{f}}\right)$. It is given by

$$
\begin{aligned}
H:= & \int d^{3} p \frac{p^{2}}{2} \eta^{*}(p) \eta(p)+\int d^{3} k|k| a^{*}(k) a(k) \\
& +\left(\int d^{3} p d^{3} k v_{\bar{\alpha}}(k) \eta^{*}(p+k) a(k) \eta(p)+\text { h.c. }\right),
\end{aligned}
$$

where $\eta^{*}(p), a^{*}(k)$ are the electron and photon creation operators and $v_{\bar{\alpha}}$ is a form factor defined in (1.29). (We refer to Sect. 1.1 for a precise definition of the model.) As $H$ preserves the number of electrons, we can restrict it to the one-electron subspace obtaining the Hamiltonian $H^{(1)}$. This Hamiltonian has the standard decomposition into the fiber Hamiltonians at fixed total momentum $H_{p}^{(1)}$, which are operators on the Fock space. In the infraredregular case $(1 / 2 \geq \bar{\alpha}>0$ in (1.29) below), the fiber Hamiltonians have (normalized) ground states $\psi_{p}$ (for $p$ in some ball $S$ centered at zero), corresponding to eigenvalues $E_{p}$. Superpositions of such ground states of the form

$$
\psi_{h}:=\Pi^{*} \int^{\oplus} d^{3} p h(p) \psi_{p}, \quad h \in C_{0}^{2}(S),
$$


give physical single-electron states in $\mathcal{H}$. (Here $\Pi^{*}$ is the standard identification between the fiber picture and the physical picture.) We note that the time evolution of $\psi_{h}$ is given by

$$
e^{-i H t} \psi_{h}=\psi_{h_{t}},
$$

where $h_{t}(p):=e^{-i E_{p} t} h(p)$. In heuristic terms, to construct a scattering state of two electrons one has to 'multiply' two single-electron states in such a way that the result is a vector in $\mathcal{H}$. In essence, Haag-Ruelle scattering theory is a prescription to perform such a multiplication. In the context of persistent models with a trivial infrared structure, for instance Nelson model with a fixed infrared cut-off, this construction has been implemented in unpublished notes of J. Fröhlich [14] and in a work of S. Albeverio [2, 3] by making use of the so called renormalized creation operator: Let $\left\{f_{p}^{m}\right\}_{m \in \mathbb{N}_{0}}$ be the $m$-photon components of $\psi_{p}$. Then (1.1) can be rewritten as follows

$$
\psi_{h}=\sum_{m=0}^{\infty} \frac{1}{\sqrt{m !}} \int d^{3} p d^{3 m} k h(p) f_{p}^{m}\left(k_{1}, \ldots, k_{m}\right) a^{*}\left(k_{1}\right) \cdots a^{*}\left(k_{m}\right) \eta^{*}(p-\underline{k}) \Omega,
$$

where $\Omega \in \mathcal{H}$ is the vacuum vector, and $\underline{k}:=k_{1}+\cdots+k_{m}$. This suggests the following definition of the renormalized creation operator

$$
\hat{\eta}^{*}(h):=\sum_{m=0}^{\infty} \frac{1}{\sqrt{m !}} \int d^{3} p d^{3 m} k h(p) f_{p}^{m}\left(k_{1}, \ldots, k_{m}\right) a^{*}\left(k_{1}\right) \cdots a^{*}\left(k_{m}\right) \eta^{*}(p-\underline{k}),
$$

which creates the physical single-electron state $\psi_{h}$ from the vacuum. Now, given $h_{1}, h_{2} \in$ $C_{0}^{2}(S)$ with disjoint velocity supports (cf. formula (1.9) below), the (outgoing) scattering state $\tilde{\Psi}_{h_{1}, h_{2}}^{+}$describing an asymptotic configuration of two independent electrons $\psi_{h_{1}}, \psi_{h_{2}}$ is given by

$$
\tilde{\Psi}_{h_{1}, h_{2}}^{+}=\lim _{t \rightarrow \infty} e^{i H t} \hat{\eta}^{*}\left(h_{1, t}\right) \hat{\eta}^{*}\left(h_{2, t}\right) \Omega,
$$

if the approximating vectors on the r.h.s. are well defined and the limit exists. In the absence of infrared problems scattering states of several electrons can and have been constructed using formula (1.5) in [2, 3, 14]. Let us now briefly recall this reasoning and assess its applicability to models with non-trivial infrared structure. This discussion will lead us to a modified definition of scattering states, given in (1.12) below, which is better suited for such models.

Let us assume for a moment that $H$ has a fixed (smooth) infrared cut-off $\bar{\sigma}$ (i.e., that the smooth function $\chi_{\kappa}$ appearing in the form factor $v_{\bar{\alpha}}$ in (1.29) vanishes in a ball of radius $\bar{\sigma}$ ) and let $\tilde{\Psi}_{t}$ be the approximants on the r.h.s. of (1.5). Making use of the fact that $\psi_{h}=\hat{\eta}^{*}(h) \Omega$ and $H \psi_{h}=\psi_{E h}$, where $(E h)(p):=E_{p} h(p)$, we obtain

$$
\partial_{t} \tilde{\Psi}_{t}=e^{i t H} \frac{1}{2} i\left[\left[H_{\mathrm{I}}^{\mathrm{a}}, \hat{\eta}^{*}\left(h_{1, t}\right)\right], \hat{\eta}^{*}\left(h_{2, t}\right)\right] \Omega
$$

where $H_{\mathrm{I}}^{\mathrm{a}}$ is the photon-annihilation part of the interaction Hamiltonian $H_{\mathrm{I}}$, given by (1.30) below (cf. Proposition 2.2). Since we employ the Cook method, the proof of convergence of $t \mapsto \tilde{\Psi}_{t}$ as $t \rightarrow \infty$ boils down to the study of the time decay of the vacuum expectation values

$$
t \mapsto\left\|\left[\left[H_{\mathrm{I}}^{\mathrm{a}}, \hat{\eta}^{*}\left(h_{1, t}\right)\right], \hat{\eta}^{*}\left(h_{2, t}\right)\right] \Omega\right\|^{2}
$$


Apart from some Fock space combinatorics and the straightforward pointwise estimate in (1.51), the control of (1.7) as $t \rightarrow \infty$ relies on the decay properties of oscillating integrals of the type

$$
\int d^{3} \tilde{r} v_{\bar{\alpha}}(\tilde{r}) e^{-i\left(E_{q+\tilde{r}}+E_{p-\tilde{r}}\right) t} h_{1}(p-\tilde{r}) h_{2}(q+\tilde{r}) f_{q+\tilde{r}}^{n+1}(r, \tilde{r}) f_{p-\tilde{r}}^{m}(k),
$$

where we employed the short-hand notation explained in (1.55). The integral in (1.8) can be estimated by the non-stationary phase method which exploits: (1) the smoothness in $q+\tilde{r}$ and $p-\tilde{r}$ of the functions $h_{1}, h_{2} ;(2)$ analyticity of the functions $f_{p}^{m}(k)$ in $p$ which holds in the presence of the fixed infrared cut-off $\bar{\sigma}$ and for the coupling constant sufficiently small depending on $\bar{\sigma}$; (3) the disjointness of the velocity supports of the functions $h_{1}, h_{2}$, defined as

$$
V\left(h_{i}\right):=\left\{\nabla E_{p} \mid p \in \operatorname{supp} h_{i}\right\}, \quad i \in\{1,2\} .
$$

This last property follows from the assumed disjointness of the supports of $h_{1}, h_{2}$ and the invertibility of the relation $S \ni p \mapsto \nabla E_{p}$.

The ideas in the procedure outlined above have been implemented in [14] for models with a fixed infrared cut-off and in [2,3] for models with massive photons. However, in the absence of the fixed infrared cut-off $\bar{\sigma}$ we lose information on the regularity properties in $p$ of the functions $f_{p}^{m}$ and their derivatives. Such properties are difficult to obtain directly due to the fact that the corresponding eigenvalue $E_{p}$ is located at the bottom of the continuous spectrum of $H_{p}^{(1)}$. Therefore, we will not construct scattering states with the help of formula (1.5), but instead we will use more tractable approximating sequences: Let $H_{p, \sigma}^{(1)}$ be the fiber Hamiltonians with an infrared cut-off $\sigma$, defined precisely in (1.38). Their ground states $\psi_{p, \sigma}$, corresponding to eigenvalues $E_{p, \sigma}$, satisfy

$$
\lim _{\sigma \rightarrow 0} \psi_{p, \sigma}=\psi_{p}
$$

Let $\left\{f_{p, \sigma}^{m}\right\}_{m \in \mathbb{N}_{0}}$ be the $m$-photon components of $\psi_{p, \sigma}$. The renormalized creation operators with the infrared cut-off $\sigma$ have the form

$$
\hat{\eta}_{\sigma}^{*}(h):=\sum_{m=0}^{\infty} \frac{1}{\sqrt{m !}} \int d^{3} p d^{3 m} k h(p) f_{p, \sigma}^{m}\left(k_{1}, \ldots, k_{m}\right) a^{*}\left(k_{1}\right) \cdots a^{*}\left(k_{m}\right) \eta^{*}(p-\underline{k}) .
$$

We will use them to construct the scattering states as follows

$$
\Psi_{h_{1}, h_{2}}^{+}:=\lim _{t \rightarrow \infty} e^{i H t} \hat{\eta}_{\sigma_{t}}^{*}\left(h_{1, t}\right) \hat{\eta}_{\sigma_{t}}^{*}\left(h_{2, t}\right) \Omega,
$$

where $\sigma_{t}=\kappa / t^{\gamma}, \gamma \in\left(4, \gamma_{0}\right]$ and $\kappa>0$ is the ultraviolet cut-off. (We consider only outgoing states as the construction of incoming states is analogous.) The existence of this limit and its properties, which allow to interpret it as an asymptotic configuration of two electrons, constitute the main result of this paper stated in Theorem 1.3 below. (A reformulation of this result in terms of wave operators can be found below this theorem). We do not expect any problems with generalizing our findings to an arbitrary number of electrons or with changing the statistics of our 'electrons' from Bose to Fermi. However, our aim here is not to cover the most general situation, but rather to prepare grounds for our future investigation of Coulomb scattering in the infrared-singular case.

In this case (i.e., infrared singular) we have, instead of (1.10), $w-\lim _{\sigma \rightarrow 0} \psi_{p, \sigma}=0$, which is a manifestation of the infraparticle problem. However, one has the existence of 
$\phi_{p}:=\lim _{\sigma \rightarrow 0} W_{P, \sigma} \psi_{p, \sigma}$, where $W_{P, \sigma}$ is a suitable Weyl operator implementing a singular Bogoliubov transformation in the limit $\sigma \rightarrow 0$ [24]. Using this fact, it is shown in [25] how an interpolating Weyl operator used in the L.S.Z. (Lehmann-Symanzik-Zimmermann) approach to scattering theory applied to a single-electron state $\psi_{\sigma_{t}}(h):=\Pi^{*} \int^{\oplus} d^{3} p h(p) \psi_{p, \sigma_{t}}$ yields a well defined vector in the Hilbert space $\mathcal{H}$ as $t \rightarrow \infty$. In fact, this vector describes an asymptotic electron, with wave function $h$, surrounded by a cloud of infinitely many asymptotic photons. Taking these results as a guide, there is no doubt that similar clouds will have to be incorporated into the approximating vectors (1.12) before the existence of the limit can be proven. We mention in this context that the spectral estimate (1.16) below (proven in the companion paper [12]), which is one of the main new ingredients of our proof, remains valid in the infrared-singular situation. We also remark that in this situation we cannot see much room for improvements of this estimate with available methods.

Let us now return to the infrared-regular case. Here substantial refinements of estimate (1.16) seem to be within reach: we expect that the factor $1 / \sigma^{\delta_{\lambda_{0}}}$ on the r.h.s. can be omitted and one can put $\sigma=0$ on both sides. With such refined spectral information one should be able to show that the limit (1.5) also exists and coincides with (1.12). Additional supporting evidence to this effect comes from algebraic quantum field theory, where the existence of scattering states in the infrared-regular situation has been proven without introducing cut-offs [11]. However, the problem of existence of (1.5) will not be treated here.

Let us now outline the new ingredients of the proof of existence of the limit in (1.12), needed in the absence of a fixed infrared cut-off. We define the auxiliary vectors $\Psi_{t, \sigma}:=$ $e^{i H t} \hat{\eta}_{\sigma}^{*}\left(h_{1, t}\right) \hat{\eta}_{\sigma}^{*}\left(h_{2, t}\right) \Omega$ and study the convergence of $t \mapsto \Psi_{t, \sigma_{t}}$ as $t \rightarrow \infty$. For $t_{2} \geq t_{1} \geq 1$ sufficiently large so that $\sigma_{t_{2}} \leq \sigma_{t_{1}} \leq 1$ we write

$$
\left\|\Psi_{t_{2}, \sigma_{t_{2}}}-\Psi_{t_{1}, \sigma_{t_{1}}}\right\| \leq\left\|\Psi_{t_{2}, \sigma_{t_{2}}}-\Psi_{t_{1}, \sigma_{t_{2}}}\right\|+\left\|\Psi_{t_{1}, \sigma_{t_{2}}}-\Psi_{t_{1}, \sigma_{t_{1}}}\right\| .
$$

We will verify the Cauchy criterion by proving that each term on the r.h.s. of (1.13) vanishes as $t_{1} \rightarrow \infty$. The second term on the r.h.s. of (1.13) can be estimated using that $\psi_{p, \sigma}$ converges to $\psi_{p}$ as $\sigma \rightarrow 0$ at a controllable rate and that $t \mapsto \sigma_{t}$ tends sufficiently fast to zero. As for the first term on the r.h.s. of (1.13), we write

$$
\Psi_{t_{2}, \sigma_{t_{2}}}-\Psi_{t_{1}, \sigma_{t_{2}}}=\int_{t_{1}}^{t_{2}} d t \partial_{t} \Psi_{t, \sigma_{t_{2}}}
$$

and compute the derivative similarly as in (1.6) above. We obtain a term with a double commutator, familiar from (1.6), and several other terms which vanish in the limit $\sigma \rightarrow 0$ (see Lemma 2.1). In Sect. 3 and in Appendix B we use Fock space combinatorics to express $\left\|\partial_{t} \Psi_{t, \sigma_{t_{2}}}\right\|^{2}$ by oscillating integrals of the form (1.8). This part does not differ substantially from the analysis of Fröhlich in [14]. However, the treatment of these oscillating integrals in the present situation, (see Sect. 4), is much more involved than in the case of a constant infrared cut-off, studied in [14]: Given the integral

$$
\int d^{3} \tilde{r} v_{\bar{\alpha}}(\tilde{r}) e^{-i\left(E_{q+\tilde{r}}+E_{p-\tilde{r}}\right) t} h_{1}(p-\tilde{r}) h_{2}(q+\tilde{r}) f_{q+\tilde{r}, \sigma_{t_{2}}}^{n+1}(r, \tilde{r}) f_{p-\tilde{r}, \sigma_{t_{2}}}^{m}(k), \quad t_{1}<t<t_{2},
$$

we first introduce an auxiliary slow (smooth) infrared cut-off at the energy scale $\kappa\left(\sigma_{t_{2}} / \kappa\right)^{1 /\left(8 \gamma_{0}\right)}$ so as to split the integration domain into two regions corresponding to $\sigma_{t_{2}}<|\tilde{r}|<\kappa\left(\sigma_{t_{2}} / \kappa\right)^{1 /\left(8 \gamma_{0}\right)}$ and $\kappa\left(\sigma_{t_{2}} / \kappa\right)^{1 /\left(8 \gamma_{0}\right)}<|\tilde{r}|<\kappa$, respectively. In the first region the decrease of the slow cut-off as $t \rightarrow \infty$ facilitates the decay of the integral so that it 
suffices to perform the integration by parts (dictated by the non-stationary phase method) only once. In this region careful attention has to be paid to boundary terms generated by the sharp cut-off $\sigma_{t_{2}}$. There are no such boundary terms in the second region which is confined between two smooth cut-offs. In the second region we have to integrate by parts twice. The ingredients (1) and (3), listed below (1.8), enter into this analysis as in the case of a fixed cut-off. However, (2) looses validity in the present situation. In order to perform the integration by parts discussed above, we employ instead bounds of the following form, stated precisely in Theorem 1.2 below,

$$
\left|\partial_{p}^{\beta} f_{p, \sigma}^{m}\left(k_{1}, \ldots, k_{m}\right)\right| \leq \frac{1}{\sqrt{m !}}\left(\frac{1}{\sigma^{\delta_{\lambda_{0}}}}\right)^{|\beta|} \frac{c v_{\bar{\alpha}}^{\sigma}\left(k_{1}\right)}{\left|k_{1}\right|} \cdots \frac{c v_{\bar{\alpha}}^{\sigma}\left(k_{m}\right)}{\left|k_{m}\right|} .
$$

Here $\beta$ is any multiindex s.t. $0 \leq|\beta| \leq 2$, the form-factor $v_{\bar{\alpha}}^{\sigma}$ is given by (1.39), $\lambda_{0}$ is the maximal admissible value of the coupling constant and the function $\lambda_{0} \rightarrow \delta_{\lambda_{0}}$ tends to zero as $\lambda_{0} \rightarrow 0$. Thus, although bound (1.16) is not uniform in $\sigma$, the dependence on the infrared cut-off is very mild in the weak coupling regime. Such estimates suffice for the purpose of completing our non-stationary phase arguments in Sect. 4.

Let us conclude this introductory discussion with some remarks concerning estimate (1.16), whose complete proof is postponed to a separate paper [12]. We recall that already in $[15,16]$ a formula for $f_{p, \sigma}^{m}$ was derived, which in the case of $m=1$ has the form

$$
f_{p, \sigma}^{1}(k)=-\left\langle\Omega,\left\{\frac{1}{H_{p-k, \sigma}^{(1)}-E_{p, \sigma}+|k|} v_{\bar{\alpha}}^{\sigma}(k)\right\} \psi_{p, \sigma}\right\rangle,
$$

where the form-factor $v_{\bar{\alpha}}^{\sigma}$ is given by (1.39). It is not difficult to obtain from this formula that

$$
\left|f_{p, \sigma}^{1}(k)\right| \leq \frac{c v_{\alpha}^{\sigma}(k)}{|k|}
$$

where the constant $c$ is independent of $\sigma$. This gives estimate (1.16) in the special case of $m=1, \beta=0$. To indicate various difficulties that have to be tackled in the case $\beta>0$, let us differentiate (1.17) w.r.t. $p$ :

$$
\begin{aligned}
\partial_{p^{i}} f_{p, \sigma}^{1}(k)= & \left\langle\Omega,\left\{\frac{1}{H_{p-k, \sigma}^{(1)}-E_{p, \sigma}+|k|}\left(\left(p-k-P_{\mathrm{f}}\right)-\nabla E_{p, \sigma}\right)^{i}\right.\right. \\
& \left.\left.\times \frac{1}{H_{p-k, \sigma}^{(1)}-E_{p, \sigma}+|k|} v_{\bar{\alpha}}^{\sigma}(k)\right\} \psi_{p, \sigma}\right\rangle \\
& -\left\langle\Omega,\left\{\frac{1}{H_{p-k, \sigma}^{(1)}-E_{p, \sigma}+|k|} v_{\bar{\alpha}}^{\sigma}(k)\right\} \partial_{p^{i}} \psi_{p, \sigma}\right\rangle,
\end{aligned}
$$

where $P_{\mathrm{f}}$ is the photon momentum operator. As for the last term on the r.h.s. of (1.19) the main difficulty is to find an estimate on the vector $\partial_{p^{i}} \psi_{p, \sigma}$ with suitable dependence on $\sigma$. We recall that the existing bounds from [24] give only Hölder continuity of $p \mapsto \psi_{p, \sigma}$, uniformly in $\sigma$. By a refinement of the argument from [24], we obtain in [12] the following bound, stated precisely in Proposition 1.1 below,

$$
\left\|\partial_{p^{i}} \psi_{p, \sigma}\right\| \leq \frac{c}{\sigma^{\delta_{\lambda_{0}}}}
$$


where $c$ is independent of $\sigma$. Denoting by $I I$ the last term on the r.h.s. of (1.19), and proceeding as in the derivation of (1.18), we obtain

$$
|I I| \leq \frac{1}{\sigma^{\delta_{\lambda_{0}}}} \frac{c v_{\bar{\alpha}}^{\sigma}(k)}{|k|} .
$$

As for the first term on the r.h.s. of (1.19), which we denote by $I$, a crude estimate gives

$$
|I| \leq \frac{c v_{\bar{\alpha}}^{\sigma}(k)}{|k|^{2}} \leq \frac{1}{\sigma} \frac{c v_{\bar{\alpha}}^{\sigma}(k)}{|k|},
$$

due to the presence of the additional resolvent and the support properties of $v_{\bar{\alpha}}^{\sigma}$. This bound is much weaker than (1.16) and does not suffice for the purpose of constructing scattering states. With the help of the multiscale analysis we improve it to

$$
|I| \leq \frac{1}{\sigma^{\delta_{\lambda_{0}}}} \frac{c v_{\bar{\alpha}}^{\sigma}(k)}{|k|},
$$

which is only slightly worse than (1.18). Altogether we get

$$
\left|\partial_{p^{i}} f_{p, \sigma}^{1}(k)\right| \leq \frac{1}{\sigma^{\delta_{\lambda_{0}}}} \frac{c v_{\bar{\alpha}}^{\sigma}(k)}{|k|},
$$

which gives a special case of (1.16) for $m=1$ and $|\beta|=1$. By differentiating (1.17) again, applying similar arguments as above and carefully exploiting cancellations of certain infrared-divergent terms we can cover also the case $|\beta|=2$. We refer the reader to Theorem 1.2 below and to [12] for more details concerning estimate (1.16).

This paper is organized as follows: In Sect. 1.1 we recall the definition of the Nelson model with many electrons. In Sect. 1.2 we state the relevant results concerning spectral theory, which are proven in a separate paper [12]. In Sect. 1.3 we state the main result of the present paper which concerns scattering theory of two electrons in the infrared-regular massless Nelson model. Section 2 presents the main steps of the proof and in the following sections we provide the necessary ingredients. Section 3 is devoted to the vacuum expectation values of the renormalized creation operators. In Sect. 4 decay properties of these vacuum expectation values are derived with the help of the method of non-stationary phase. Important input in this section are our spectral results proven in [12] and summarized in Sect. 1.2. The more technical part of our discussion is postponed to the appendices.

\subsection{The Model}

We consider an interacting system of massive spinless bosons, which we call 'electrons', and massless spinless bosons, which we will call 'photons'. Let $\mathfrak{h}_{\mathrm{e}}:=L^{2}\left(\mathbb{R}^{3}, d^{3} p\right)$ and $\mathfrak{h}_{\mathrm{f}}:=L^{2}\left(\mathbb{R}^{3}, d^{3} k\right)$ be the single-electron and single-photon spaces, respectively, and let $\Gamma\left(\mathfrak{h}_{\mathrm{e}}\right)$ and $\Gamma\left(\mathfrak{h}_{\mathrm{f}}\right)$ be the corresponding symmetric Fock spaces. The (improper) creation and annihilation operators on $\Gamma\left(\mathfrak{h}_{\mathrm{e}}\right)$ (resp. $\Gamma\left(\mathfrak{h}_{\mathrm{f}}\right)$ ) will be denoted by $\eta^{*}(p), \eta(p)$ (resp. $\left.a^{*}(k), a(k)\right)$. They satisfy the canonical commutation relations:

$$
\begin{aligned}
& {\left[\eta(p), \eta^{*}\left(p^{\prime}\right)\right]=\delta\left(p-p^{\prime}\right), \quad\left[\eta(p), \eta\left(p^{\prime}\right)\right]=\left[\eta^{*}(p), \eta^{*}\left(p^{\prime}\right)\right]=0,} \\
& {\left[a(k), a^{*}\left(k^{\prime}\right)\right]=\delta\left(k-k^{\prime}\right), \quad\left[a(k), a\left(k^{\prime}\right)\right]=\left[a^{*}(k), a^{*}\left(k^{\prime}\right)\right]=0 .}
\end{aligned}
$$


The free Hamiltonians of the electrons and photons are given by

$$
H_{\mathrm{e}}:=\int d^{3} p \Omega(p) \eta^{*}(p) \eta(p), \quad H_{\mathrm{f}}:=\int d^{3} k \omega(k) a^{*}(k) a(k),
$$

where $\Omega(p)=\frac{p^{2}}{2}$ and $\omega(k)=|k|$. We recall that these operators are essentially self-adjoint on $\mathcal{C}_{\mathrm{e}}, \mathcal{C}_{\mathrm{f}}$, respectively, where $\mathcal{C}_{\mathrm{e} / \mathrm{f}} \subset \Gamma\left(\mathfrak{h}_{\mathrm{e} / \mathrm{f}}\right)$ are dense subspaces consisting of finite linear combinations of symmetrized tensor products of elements of $C_{0}^{\infty}\left(\mathbb{R}^{3}\right)$.

The physical Hilbert space of our system is $\mathcal{H}:=\Gamma\left(\mathfrak{h}_{\mathrm{e}}\right) \otimes \Gamma\left(\mathfrak{h}_{\mathrm{f}}\right)$ and we will follow the standard convention to denote operators of the form $A \otimes 1$ and $1 \otimes B$ by $A$ and $B$, respectively. The Hamiltonian describing the free evolution of the composite system of electrons and photons is given by

$$
H_{\mathrm{fr}}:=H_{\mathrm{e}}+H_{\mathrm{f}}
$$

and it is essentially self-adjoint on $\mathcal{C}:=\mathcal{C}_{\mathrm{e}} \otimes \mathcal{C}_{\mathrm{f}}$.

Now let us introduce the interaction between electrons and photons. Let $\lambda>0$ be the coupling constant, $\kappa=1$ be the ultraviolet cut-off ${ }^{1}$ and let $1 / 2 \geq \bar{\alpha} \geq 0$ be a parameter which controls the infrared behavior of the system. Given these parameters, we define the form-factor

$$
v_{\bar{\alpha}}(k):=\lambda \frac{\chi_{\kappa}(k)|k|^{\bar{\alpha}}}{(2|k|)^{\frac{1}{2}}},
$$

where $\chi_{\kappa} \in C_{0}^{\infty}\left(\mathbb{R}^{3}\right)$ is rotationally invariant, non-increasing in the radial direction, supported in $\mathcal{B}_{\kappa}$ and equal to one on $\mathcal{B}_{\left(1-\varepsilon_{0}\right) \kappa}$, for some fixed $0<\varepsilon_{0}<1$. (We denote by $\mathcal{B}_{r}$ the open ball of radius $r$ centered at zero.) The interaction Hamiltonian, defined as a symmetric operator on $\mathcal{C}$, is given by the following formula

$$
H_{\mathrm{I}}:=\int d^{3} p d^{3} k v_{\bar{\alpha}}(k) \eta^{*}(p+k) a(k) \eta(p)+\text { h.c. }
$$

For future reference we denote by $H_{\mathrm{I}}^{\mathrm{a}}$ the first term on the r.h.s. of $(1.30)$ and set $H_{\mathrm{I}}^{\mathrm{c}}:=$ $\left(H_{\mathrm{I}}^{\mathrm{a}}\right)^{*}$.

As indicated in $[15,16]$, the full Hamiltonian $H:=H_{\mathrm{fr}}+H_{\mathrm{I}}$ can be defined as a selfadjoint operator on a dense domain in $\mathcal{H}$. For the reader's convenience we outline briefly this construction: First, we note that both $H_{\mathrm{fr}}$ and $H_{\mathrm{I}}$ preserve the number of electrons. Let us therefore define $\mathcal{H}^{(n)}:=\Gamma^{(n)}\left(\mathfrak{h}_{\mathrm{e}}\right) \otimes \Gamma\left(\mathfrak{h}_{\mathrm{f}}\right)$, where $\Gamma^{(n)}\left(\mathfrak{h}_{\mathrm{e}}\right)$ is the $n$-particle subspace of $\Gamma\left(\mathfrak{h}_{\mathrm{e}}\right)$ and let $H_{\mathrm{fr}}^{(n)}$ and $H_{\mathrm{I}}^{(n)}$ be the restrictions of the respective operators to $\mathcal{H}^{(n)}$, defined on $\mathcal{C}^{(n)}:=\mathcal{C} \cap \mathcal{H}^{(n)}$. As shown in Lemma A.1, using the Kato-Rellich theorem, each $H^{(n)}=$ $H_{\mathrm{fr}}^{(n)}+H_{\mathrm{I}}^{(n)}$ can be defined as a bounded from below, self-adjoint operator on the domain of $H_{\mathrm{fr}}^{(n)}$, which is essentially self-adjoint on $\mathcal{C}^{(n)}$. Then we can define

$$
H:=\bigoplus_{n \in \mathbb{N}_{0}} H^{(n)}
$$

as an operator on $\mathcal{C}$. Since $H^{(n)} \pm i$ have dense ranges on $\mathcal{C}^{(n)}, H \pm i$ have dense ranges on $\mathcal{C}$, thus $H$ is essentially self-adjoint on this domain. We stress that the above construction is

\footnotetext{
${ }^{1}$ We set $\kappa=1$ to simplify the proofs of Proposition 1.1 and Theorem 1.2, given in the companion paper [12]. In the present paper we will write $\kappa$ explicitly.
} 
valid both in the infrared-regular case $(1 / 2 \geq \bar{\alpha}>0)$ and in the infrared-singular situation $(\bar{\alpha}=0)$.

On $\mathcal{C}$ we have the following formula for $H$

$$
\begin{aligned}
H= & \int d^{3} p \Omega(p) \eta^{*}(p) \eta(p)+\int d^{3} k \omega(k) a^{*}(k) a(k) \\
& +\left(\int d^{3} p d^{3} k v_{\bar{\alpha}}(k) \eta^{*}(p+k) a(k) \eta(p)+\text { h.c. }\right) .
\end{aligned}
$$

It reduces to a more familiar expression on $\mathcal{C}^{(n)}$

$$
H^{(n)}=\sum_{i=1}^{n} \frac{\left(i \nabla_{x_{i}}\right)^{2}}{2}+\int d^{3} k \omega(k) a^{*}(k) a(k)+\sum_{i=1}^{n} \int d^{3} k v_{\bar{\alpha}}(k)\left(e^{i k x_{i}} a(k)+e^{-i k x_{i}} a^{*}(k)\right),
$$

where $x_{i}$ is the position operator of the $i$-th electron. Finally, we introduce the electron and photon momentum operators

$$
P_{\mathrm{e}}^{i}:=\int d^{3} p p^{i} \eta^{*}(p) \eta(p), \quad P_{\mathrm{f}}^{i}:=\int d^{3} k k^{i} a^{*}(k) a(k), \quad i \in\{1,2,3\},
$$

which are essentially self-adjoint on $\mathcal{C}$. We recall that $H$ is translationally invariant, that is it commutes with the total momentum operators $P^{i}$, given by

$$
P^{i}:=P_{\mathrm{e}}^{i}+P_{\mathrm{f}}^{i}, \quad i \in\{1,2,3\},
$$

which are essentially self-adjoint on $\mathcal{C}$ as well.

\subsection{Spectral Theory}

In this section we collect some necessary information about the spectrum of the singleelectron Hamiltonian $H^{(1)}$. We recall that the analysis of the spectrum of $H_{p}^{(1)}$ was initiated in $[15,16]$ and advanced in [24] with the help of iterative analytic perturbation theory. Further developments along these lines, which we describe in detail below, can be found in [12]. Interesting results on the spectrum of the Nelson model with a slightly different form factor were also obtained in [1] by different methods.

We recall that due to the translational invariance $H^{(1)}$ can be decomposed into a direct integral of the fiber Hamiltonians $H_{p}^{(1)}$ as follows

$$
H^{(1)}=\Pi^{*} \int^{\oplus} d^{3} p H_{p}^{(1)} \Pi
$$

Here $\Pi: \mathcal{H}^{(1)} \rightarrow L^{2}\left(\mathbb{R}^{3} ; \Gamma\left(\mathfrak{h}_{\mathrm{fi}}\right)\right)$ is a unitary map given by $\Pi:=F e^{i P_{\mathrm{f}} x}$, where $x^{i}, i=1,2$, 3 are the components of the electron's position operator and $F$ is the Fourier transform in the electron's variables. The Hamiltonians $H_{p}^{(1)}$ are self-adjoint operators on the Fock space $\Gamma\left(\mathfrak{h}_{\mathrm{fi}}\right)$, where $\mathfrak{h}_{\mathrm{fi}}=L^{2}\left(\mathbb{R}^{3}, d^{3} k\right)$ is the single-photon space in the fiber picture. Denoting by $b^{*}(k)$ and $b(k)$ the creation and annihilation operators on $\Gamma\left(\mathfrak{h}_{\mathrm{fi}}\right)$ one easily obtains from (1.33) that

$$
H_{p}^{(1)}=\frac{1}{2}\left(p-P_{\mathrm{f}}\right)^{2}+H_{\mathrm{f}}+\int d^{3} k v_{\bar{\alpha}}(k)\left(b(k)+b^{*}(k)\right) .
$$


As a tool to study the spectrum of this Hamiltonian, we introduce auxiliary fiber Hamiltonians with infrared cut-offs

$$
H_{p, \sigma}^{(1)}=\frac{1}{2}\left(p-P_{\mathrm{f}}\right)^{2}+H_{\mathrm{f}}+\int d^{3} k v_{\bar{\alpha}}^{\sigma}(k)\left(b(k)+b^{*}(k)\right) .
$$

The form-factor $v_{\bar{\alpha}}^{\sigma}$ is defined as follows

$$
v_{\bar{\alpha}}^{\sigma}(k):=\lambda \frac{\chi_{[\sigma, \kappa)}(k)|k|^{\bar{\alpha}}}{(2|k|)^{\frac{1}{2}}},
$$

where $0<\sigma \leq \kappa, \chi_{[\sigma, \kappa)}(k):=\mathbf{1}_{\mathcal{B}_{\sigma}^{\prime}}(k) \chi_{\kappa}(k), \mathcal{B}_{\sigma}^{\prime}$ is the complement of the ball of radius $\sigma$ and $\mathbf{1}_{\Delta}$ is the characteristic function of a set $\Delta$. By replacing $v_{\bar{\alpha}}$ with $v_{\bar{\alpha}}^{\sigma}$ in (1.30), we obtain the interaction Hamiltonian $H_{\mathrm{I}, \sigma}$ and the corresponding full Hamiltonian $H_{\sigma}$ with an infrared cut-off. The restriction of $H_{\sigma}$ to the single-electron subspace, denoted by $H_{\sigma}^{(1)}$, has a fiber decomposition into the Hamiltonians $H_{p, \sigma}^{(1)}$.

Since we are particularly interested in the bottom of the spectrum of $H_{p}^{(1)}$ and $H_{p, \sigma}^{(1)}$, let us define

$$
E_{p}:=\inf \sigma\left(H_{p}^{(1)}\right), \quad E_{p, \sigma}:=\inf \sigma\left(H_{p, \sigma}^{(1)}\right) .
$$

As the model is non-relativistic, we restrict attention to small values of the total momentum $p$ at which the electron moves slower than the photons. More precisely, we consider $p$ from the set

$$
S:=\left\{p \in \mathbb{R}^{3}|| p \mid<p_{\max }\right\}
$$

for some $p_{\max }>0$. Since we work in the weak coupling regime, we fix some sufficiently small $\lambda_{0}>0$, and restrict attention to $\lambda \in\left(0, \lambda_{0}\right]$. The parameters $p_{\max }$ and $\lambda_{0}$ are specified in Proposition 1.1 and Theorem 1.2 below. $p_{\max }$ remains fixed in the course of our analysis. The maximal coupling constant $\lambda_{0}$ is readjusted only in the last step of our investigation - in Theorem 2.1 - to a new value which is denoted by $\lambda_{0}^{\prime}$.

In the following proposition we collect the results concerning $E_{p}, E_{p, \sigma}$ which will be needed in the present investigation. Some of these properties are known, but several are new, as we explain below.

Proposition 1.1 Fix $0 \leq \bar{\alpha} \leq 1 / 2$ and let $p_{\max }=1 / 6$. Then there exists $\lambda_{0}>0$ s.t. for all $p \in S:=\mathcal{B}_{p_{\max }}, \lambda \in\left(0, \lambda_{0}\right]$ there holds:

(a) $S \ni p \mapsto E_{p}$ is twice continuously differentiable and strictly convex. $S \ni p \mapsto E_{p, \sigma}$ is analytic and strictly convex, uniformly in $\sigma \in(0, \kappa]$ (i.e., the minimal distance of the spectrum of $\nabla^{2} E_{p, \sigma}$ from zero is larger than some $c>0, c$ independent of $\left.\sigma\right)$. Moreover,

$$
\begin{aligned}
& \left|E_{p}-E_{p, \sigma}\right| \leq c \sigma, \\
& \left|\partial_{p}^{\beta_{1}} E_{p, \sigma}\right| \leq c, \quad\left|\partial_{p}^{\beta_{2}} E_{p, \sigma}\right| \leq c, \quad\left|\partial_{p}^{\beta_{3}} E_{p, \sigma}\right| \leq c / \sigma^{\delta_{\lambda_{0}}}
\end{aligned}
$$

for multiindices $\beta_{j}$ s.t. $\left|\beta_{j}\right|=j, j \in\{1,2,3\}$.

(b) For $\sigma>0, E_{p, \sigma}$ is a simple eigenvalue corresponding to a normalized eigenvector $\psi_{p, \sigma}$, whose phase is specified in [12]. There holds

$$
\left\|\partial_{p}^{\beta} \psi_{p, \sigma}\right\| \leq c / \sigma^{\delta_{\lambda_{0}}}
$$

for multiindices $\beta$ s.t. $0<|\beta| \leq 2$. 
(c) For $\bar{\alpha}>0, E_{p}$ is a simple eigenvalue corresponding to a normalized eigenvector $\psi_{p}$. Moreover, for a suitable choice of the phase of $\psi_{p}$,

$$
\left\|\psi_{p}-\psi_{p, \sigma}\right\| \leq c \sigma^{\bar{\alpha}} .
$$

The constant $c$ above is independent of $\sigma, p, \lambda, \bar{\alpha}$ within the assumed restrictions. Clearly, all statements above remain true after replacing $\lambda_{0}$ by some $\tilde{\lambda}_{0} \in\left(0, \lambda_{0}\right]$. The resulting function $\tilde{\lambda}_{0} \mapsto \delta_{\tilde{\lambda}_{0}}$ can be chosen positive and s.t. $\lim _{\tilde{\lambda}_{0} \rightarrow 0} \delta_{\tilde{\lambda}_{0}}=0$.

In the light of Proposition 1.1 we can define the subspace of renormalized single-electron states

$$
\mathcal{H}_{1, \sigma}:=\left\{\Pi^{*} \int^{\oplus} d^{3} p h(p) \psi_{p, \sigma} \mid h \in L^{2}\left(\mathbb{R}^{3}, d^{3} p\right), \operatorname{supp} h \subset S\right\} .
$$

In the case of $1 / 2 \geq \bar{\alpha}>0$ we also set $\mathcal{H}_{1}:=\mathcal{H}_{1, \sigma=0}$.

Large part of Proposition 1.1 has already been established in the Nelson model or in similar models: The fact that $S \ni p \mapsto E_{p}$ is twice continuously differentiable and convex has been shown in non-relativistic and semi-relativistic QED in $[4,17,23]$ and in the Nelson model with a slightly different form-factor in [1]. The present case is covered by our analysis in [12]. The bound in (1.42) can be extracted from [24]. The first sentence in Proposition 1.1(b) has been established already in [16]. Part (c) is implicit in [24] and is shown explicitly in [12]. The bound on the third derivative of $E_{p, \sigma}$ in (1.43) and on the first and second derivative of $\psi_{p, \sigma}$ in (1.44) are new and are proven in [12].

It turns out that the properties stated in Proposition 1.1 are not quite enough for our purposes. Scattering theory for several electrons requires much more detailed information about the electron's localization in space than scattering of one electron and photons. This information is contained in regularity properties of the momentum wave functions of the vectors $\psi_{p, \sigma}$. Let us express $\psi_{p, \sigma}$ in terms of its $m$-particle components in the Fock space:

$$
\psi_{p, \sigma}=\left\{f_{p, \sigma}^{m}\right\}_{m \in \mathbb{N}_{0}},
$$

where $f_{p, \sigma}^{m} \in L_{\text {sym }}^{2}\left(\mathbb{R}^{3 m}, d^{3 m} k\right)$, i.e., each $f_{p, \sigma}^{m}$ is a square-integrable function symmetric in $m$ variables from $\mathbb{R}^{3}$. Let us introduce the following auxiliary functions:

$$
g_{\sigma}^{m}\left(k_{1}, \ldots, k_{m}\right):=\prod_{i=1}^{m} \frac{c \lambda \chi_{\left[\sigma, \kappa_{*}\right)}\left(k_{i}\right)\left|k_{i}\right|^{\bar{\alpha}}}{\left|k_{i}\right|^{3 / 2}}, \quad \kappa_{*}:=\left(1-\varepsilon_{0}\right)^{-1} \kappa, 0<\varepsilon_{0}<1,
$$

where $\varepsilon_{0}$ appeared below (1.29) and $c$ is some positive constant independent of $m, \sigma, p$ and $\lambda$ within the restrictions specified above. (Note that $\chi_{\left[\sigma, \kappa_{*}\right)}(k)=1$ for $k \in[\sigma, \kappa)$ ). Finally, we introduce the notation

$$
\mathcal{A}_{r_{1}, r_{2}}:=\left\{k \in \mathbb{R}^{3}\left|r_{1}<\right| k \mid<r_{2}\right\},
$$

where $0 \leq r_{1}<r_{2}$. Now we are ready to state the required properties of the functions $f_{p, \sigma}^{m}$ :

Theorem 1.2 Fix $0 \leq \bar{\alpha} \leq 1 / 2$, set $p_{\max }=1 / 6$ and recall that $\sigma>0$. Then there exists $\lambda_{0}>0$ s.t. for all $p \in S=\mathcal{B}_{p_{\max }}, \lambda \in\left(0, \lambda_{0}\right]$ the following statements are true:

(a) Let $\left\{f_{p, \sigma}^{m}\right\}_{m \in \mathbb{N}_{0}}$ be the $m$-particle components of $\psi_{p, \sigma}$ and let $\overline{\mathcal{A}}_{\sigma, \kappa}^{\times m}$ be defined by (1.49).

Then, for any $p \in S$, the function $f_{p, \sigma}^{m}$ is supported in $\overline{\mathcal{A}}_{\sigma, \kappa}^{\times m}$. 
(b) The function

$$
S \times \mathcal{A}_{\sigma, \infty}^{\times m} \ni\left(p ; k_{1}, \ldots, k_{m}\right) \mapsto f_{p, \sigma}^{m}\left(k_{1}, \ldots, k_{m}\right)
$$

is twice continuously differentiable and extends by continuity, together with its derivatives, to the set $S \times \overline{\mathcal{A}}_{\sigma, \infty}^{\times m}$.

(c) For any multiindex $\beta, 0 \leq|\beta| \leq 2$, the function (1.50) satisfies

$$
\begin{aligned}
\left|\partial_{k_{l}}^{\beta} f_{p, \sigma}^{m}\left(k_{1}, \ldots, k_{m}\right)\right| & \leq \frac{1}{\sqrt{m !}}\left|k_{l}\right|^{-|\beta|} g_{\sigma}^{m}\left(k_{1}, \ldots, k_{m}\right), \\
\left|\partial_{p}^{\beta} f_{p, \sigma}^{m}\left(k_{1}, \ldots, k_{m}\right)\right| & \leq \frac{1}{\sqrt{m !}}\left(\frac{1}{\sigma^{\delta_{\lambda_{0}}}}\right)^{|\beta|} g_{\sigma}^{m}\left(k_{1}, \ldots, k_{m}\right), \\
\left|\partial_{p^{i}} \partial_{k_{l}^{i}} f_{p, \sigma}^{m}\left(k_{1}, \ldots, k_{m}\right)\right| & \leq \frac{1}{\sqrt{m !}} \frac{1}{\sigma^{\delta_{\lambda_{0}}}}\left|k_{l}\right|^{-1} g_{\sigma}^{m}\left(k_{1}, \ldots, k_{m}\right),
\end{aligned}
$$

where the function $\tilde{\lambda}_{0} \mapsto \delta_{\tilde{\lambda}_{0}}$ has the properties specified in Proposition 1.1.

Parts (a), (b) of Theorem 1.2 and estimate (1.51) in (c) can be extracted from [14-16] or proven using the methods from these papers. The key new input are the bounds in (1.52) and (1.53) on the first and second derivative w.r.t. $p$ with their mild dependence on the infrared cut-off $\sigma$. These bounds require major refinements of the iterative multi-scale analysis from [24]. They constitute the main technical result of the companion paper [12].

\subsection{Scattering Theory}

In this section we outline the construction of two-electron scattering states along the lines of Haag-Ruelle scattering theory [21, 27], following some ideas from [2, 3, 14]. Since we are interested here in the infrared-regular situation, we set $1 / 2 \geq \bar{\alpha}>0$. Such $\bar{\alpha}$ will be kept fixed in the remaining part of the paper. (The infrared-singular case $\bar{\alpha}=0$ is much more involved due to the infraparticle problem and will be studied elsewhere).

Let $C_{0}^{2}(S)$ be the class of twice continuously differentiable functions with compact support contained in $S$. Following [2, 3, 14], for any $h \in C_{0}^{2}(S)$ we define the renormalized creation operator

$$
\hat{\eta}_{\sigma}^{*}(h):=\sum_{m=0}^{\infty} \frac{1}{\sqrt{m !}} \int d^{3} p d^{3 m} k h(p) f_{p, \sigma}^{m}(k) a^{*}(k)^{m} \eta^{*}(p-\underline{k}) .
$$

In this expression we use the following short-hand notation, which will appear frequently below:

$$
\begin{aligned}
& f_{p, \sigma}^{m}(k):=f_{p, \sigma}^{m}\left(k_{1}, \ldots, k_{m}\right), \\
& a^{*}(k)^{m}:=a^{*}\left(k_{1}\right) \cdots a^{*}\left(k_{m}\right), \\
& \underline{k}:=k_{1}+\cdots+k_{m} .
\end{aligned}
$$

It is shown in Lemma A.2 that $\hat{\eta}_{\sigma}^{*}(h)$ and $\hat{\eta}_{\sigma}^{*}\left(h_{1}\right) \hat{\eta}_{\sigma}^{*}\left(h_{2}\right)$, for $h_{1}, h_{2} \in C_{0}^{2}(S)$, are well defined operators on $\mathcal{C}$. (Since $\hat{\eta}_{\sigma}(h):=\left(\hat{\eta}_{\sigma}^{*}(h)\right)^{*}$ is obviously well defined on $\mathcal{C}$, we obtain that $\hat{\eta}_{\sigma}^{*}(h)$ is closable). 
Now let $\Omega_{\mathrm{e}}$ and $\Omega_{\mathrm{f}}$ be the vacuum vectors of $\Gamma\left(\mathfrak{h}_{\mathrm{e}}\right)$ and $\Gamma\left(\mathfrak{h}_{\mathrm{f}}\right)$, respectively. Then $\Omega:=$ $\Omega_{\mathrm{e}} \otimes \Omega_{\mathrm{f}}$ is the physical vacuum in $\mathcal{H}$. It is easy to see that (cf. Lemma 2.3)

$$
\psi_{h, \sigma}:=\hat{\eta}_{\sigma}^{*}(h) \Omega=\Pi^{*} \int^{\oplus} d^{3} p h(p) \psi_{p, \sigma},
$$

i.e., $\psi_{h, \sigma}$ is an element of the subspace of renormalized single-electron states $\mathcal{H}_{1, \sigma}$, defined in (1.46). Since we assumed that $1 / 2 \geq \bar{\alpha}>0$, we obtain from Proposition 1.1(c) that there exists the limit

$$
\psi_{h}:=\lim _{\sigma \rightarrow 0} \hat{\eta}_{\sigma}^{*}(h) \Omega=\Pi^{*} \int^{\oplus} d^{3} p h(p) \psi_{p} .
$$

Clearly, $\psi_{h}$ belongs to the renormalized single-electron space $\mathcal{H}_{1}$ of the Hamiltonian $H$.

Let us now proceed to the construction of two-electron scattering states. We fix some parameter $\gamma_{0}>4$, which will be kept fixed in our investigation, choose some $\gamma \in\left(4, \gamma_{0}\right]$ and introduce a time-dependent cut-off

$$
\sigma_{t}:=\kappa / t^{\gamma}
$$

for $t \geq \max \{1, \kappa\}$. Next, we choose $h_{1}, h_{2} \in C_{0}^{2}(S)$ with disjoint supports and set

$$
h_{i, t}(p):=e^{-i E_{p} t} h_{i}(p), \quad i \in\{1,2\} .
$$

Now we are ready to define the two-electron scattering states approximants:

$$
\Psi_{t, h_{1}, h_{2}}:=e^{i H t} \hat{\eta}_{\sigma_{t}}^{*}\left(h_{1, t}\right) \hat{\eta}_{\sigma_{t}}^{*}\left(h_{2, t}\right) \Omega
$$

We will show that the limit of $\Psi_{t, h_{1}, h_{2}}$ exists as $t \rightarrow \infty$ and can be interpreted as a physical state describing two independent excitations. This is the content of our main result concerning scattering theory, stated below.

Theorem 1.3 Fix $1 / 2 \geq \bar{\alpha}>0, \gamma_{0}>4$. Let $\lambda \in\left(0, \lambda_{0}^{\prime}\right]$, where $\lambda_{0}^{\prime}>0$ is sufficiently small. Then, for $h_{1}, h_{2} \in C_{0}^{2}(S)$ with disjoint supports, the following statements hold:

(a) Let $\sigma_{t}=\kappa / t^{\gamma}$, where $\gamma \in\left(4, \gamma_{0}\right]$. Then there exists the limit

$$
\Psi_{h_{1}, h_{2}}^{+}:=\lim _{t \rightarrow \infty} e^{i H t} \hat{\eta}_{\sigma_{t}}^{*}\left(h_{1, t}\right) \hat{\eta}_{\sigma_{t}}^{*}\left(h_{2, t}\right) \Omega
$$

and it is called the two-electron scattering state. It is independent of the parameter $\gamma$ within the above restrictions.

(b) Let $\Psi_{h_{1}, h_{2}}^{+}, \Psi_{h_{1}^{\prime}, h_{2}^{\prime}}^{+}$be two scattering states. Their scalar product has the form

$$
\left\langle\Psi_{h_{1}, h_{2}}^{+}, \Psi_{h_{1}^{\prime}, h_{2}^{\prime}}^{+}\right\rangle=\left\langle\psi_{h_{1}}, \psi_{h_{1}^{\prime}}\right\rangle\left\langle\psi_{h_{2}}, \psi_{h_{2}^{\prime}}\right\rangle+\left\langle\psi_{h_{1}}, \psi_{h_{2}^{\prime}}\right\rangle\left\langle\psi_{h_{2}}, \psi_{h_{1}^{\prime}}\right\rangle
$$

Proof Part (a) follows from Theorem 2.1. Assumptions (2.2), (2.3) of Theorem 2.1 are verified in Proposition 3.4. Assumption 2.4 follows from Propositions 2.2, 3.1, 3.6 and Corollary 3.5. Part (b) of the theorem follows from Proposition 3.4 and Proposition 1.1(c).

In order to elucidate the meaning of Theorem 1.3, in particular of the clustering relation (1.63), let us restate it in the language of wave operators. First, we note that the vector $\psi_{h}$, given by (1.59), is determined uniquely by the function $h \in C_{0}^{2}(S)$. Moreover, vectors of 
the form $\psi_{h_{1}} \otimes_{\mathrm{s}} \psi_{h_{2}}$, where $h_{1}, h_{2} \in C_{0}^{2}(S)$ have disjoint supports, and $\otimes_{\mathrm{s}}$ is the symmetric tensor product, span a dense subspace in $\mathcal{H}_{1} \otimes_{\mathrm{s}} \mathcal{H}_{1}$. Thus we can define the wave operator $W^{+}: \mathcal{H}_{1} \otimes_{\mathrm{s}} \mathcal{H}_{1} \rightarrow \mathcal{H}$ by the following relation (and linearity)

$$
W^{+}\left(\psi_{h_{1}} \otimes_{\mathrm{s}} \psi_{h_{2}}\right):=\Psi_{h_{1}, h_{2}}^{+} .
$$

Theorem 1.3(b) says that this operator exists and extends to an isometry, similarly as in quantum mechanical scattering theory.

\section{Standing assumptions and conventions:}

1. We will only consider outgoing asymptotic configurations $(t \rightarrow \infty)$, since the incoming case $(t \rightarrow-\infty)$ is analogous. The corresponding asymptotic quantities will be denoted by an upper index + , for example $\Psi_{h_{1}, h_{2}}^{+}$in (1.63).

2. The parameters $p_{\max }=1 / 6$ and $1 / 2 \geq \bar{\alpha}>0$ are kept fixed in the remaining part of the paper.

3. As specified in Proposition 1.1 and Theorem 1.2, the maximal coupling constant $\lambda_{0}>0$ corresponds to the values of $p_{\max }$ and $1 / 2 \geq \bar{\alpha}>0$ fixed above. It remains unchanged in Sects. 3 and 4. It is readjusted to a possibly smaller value $\lambda_{0}^{\prime}>0$ in the last step of the analysis in Theorem 2.1. $\lambda_{0}^{\prime}$ may depend of $\gamma_{0}$ but not on $\gamma \in\left(4, \gamma_{0}\right]$.

4. $\tilde{\lambda}_{0} \mapsto \delta_{\tilde{\lambda}_{0}}, \tilde{\lambda}_{0} \mapsto \delta_{\tilde{\lambda}_{0}}^{\prime}$ will denote positive functions of $\tilde{\lambda}_{0} \in\left(0, \lambda_{0}\right]$, which may differ from line to line, and have the property

$$
\lim _{\tilde{\lambda}_{0} \rightarrow 0} \delta_{\tilde{\lambda}_{0}}=0, \quad \lim _{\tilde{\lambda}_{0} \rightarrow 0} \delta_{\tilde{\lambda}_{0}}^{\prime}=0 .
$$

Such functions control the infrared behaviour of our estimates listed in the statement of Theorem 2.1. By reducing the maximal coupling constant from $\lambda_{0}$ to $\lambda_{0}^{\prime}$ we make this behaviour sufficiently mild.

5. We denote by $\gamma \in\left(4, \gamma_{0}\right]$ the parameter which controls the time dependence of the (fast) infrared cut-off, i.e., $\sigma_{t}=\kappa / t^{\gamma}$. The parameter $\gamma_{0}$ is kept fixed in the remaining part of the paper. This parameter appears also in the definition of the slow infrared cut-off $\sigma_{\mathrm{s}}=\kappa(\sigma / \kappa)^{1 /\left(8 \gamma_{0}\right)}$ in the proofs of Lemmas 4.1 and 4.2 .

6. We will denote by $c, c^{\prime}, c^{\prime \prime}$ numerical constants which may depend on $S, \lambda_{0}, \varepsilon_{0}, \kappa, \bar{\alpha}$, $\gamma_{0}$ and functions $h_{1}, h_{2}$ but not on $\sigma, t$ or the electron and photon momenta. The values of these constants may change from line to line.

7. We will denote by $(p, q) \mapsto D(p, q),(p, q) \mapsto D^{\prime}(p, q)$ smooth, compactly supported functions on $\mathbb{R}^{3} \times \mathbb{R}^{3}$, which may depend of $S, \lambda_{0}, \varepsilon_{0}, \kappa, \bar{\alpha}$ but not on $\sigma, t$ or the electron and the photon momenta.

8. We will denote by $k=\left(k_{1}, \ldots, k_{m}\right) \in \mathbb{R}^{3 m}$ a collection of photon variables. A lower or upper index $m$ of a function indicates that it is a symmetric function of $\left(k_{1}, \ldots, k_{m}\right)$. For example:

$$
f^{m}(k):=f^{m}\left(k_{1}, \ldots, k_{m}\right) .
$$

Similarly, we set $a^{*}(k)^{m}:=a^{*}\left(k_{1}\right) \cdots a^{*}\left(k_{m}\right)$. We note that the order in which the components of $k$ are listed is irrelevant, since they enter always into symmetric expressions.

9. We separate the electron variable $p \in \mathbb{R}^{3}$ and the photon variables $k \in \mathbb{R}^{3 m}$ by a semicolon. For example:

$$
G_{m}(p ; k):=G_{m}\left(p ; k_{1}, \ldots, k_{m}\right) .
$$


10. Two collections of electron and photon variables $p \in \mathbb{R}^{3}, k \in \mathbb{R}^{3 m}$ and $q \in \mathbb{R}^{3}, r \in \mathbb{R}^{3 n}$ are separated by a bar. For example

$$
F_{m, n}(p ; k \mid q ; r):=F_{m, n}\left(p ; k_{1}, \ldots, k_{m} \mid q ; r_{1}, \ldots, r_{n}\right) .
$$

11. Given $k=\left(k_{1}, \ldots, k_{m}\right)$ we write $\underline{k}:=k_{1}+\cdots+k_{m}$.

\section{Main Ingredients of the Proof}

Theorem 2.1 below gives the existence of scattering states based on some assumptions proven in the later part of this paper: (a) Property (2.2)-(2.3) follows from Proposition 3.4; (b) To establish property (2.4) we derive a formula for time-derivatives of scattering state approximants in Proposition 2.2 in the later part of this section. The section concludes with Lemma 2.3 which gives a direct integral representation of the vectors $\psi_{h, \sigma}=\hat{\eta}_{\sigma}^{*}(h) \Omega$. We used this result already in (1.54) above.

In our proof of convergence of the scattering state approximants (1.62) we will vary the time $t$ and the infrared cut-off $\sigma$ independently. Let us therefore fix $h_{1}, h_{2} \in C_{0}^{2}(S)$ with disjoint supports and introduce an auxiliary two-parameter sequence

$$
\Psi_{t, \sigma}:=e^{i H t} \hat{\eta}_{\sigma}^{*}\left(h_{1, t}\right) \hat{\eta}_{\sigma}^{*}\left(h_{2, t}\right) \Omega .
$$

Now we are ready to state and prove the main result of this section.

Theorem 2.1 Let $h_{1}, h_{2} \in C_{0}^{2}(S)$ have disjoint supports, let $\Psi_{t, \sigma}$ be given by (2.1). Suppose that for $\lambda \in\left(0, \lambda_{0}\right]$, infrared cut-offs $\sigma, \sigma^{\prime}$ s.t. $\sigma \leq \sigma^{\prime} \leq \kappa$ and $t \geq \max \{1, \kappa\}$

$$
\begin{aligned}
\left\langle\Psi_{t, \sigma^{\prime}}, \Psi_{t, \sigma}\right\rangle= & \left\langle\psi_{h_{1}, \sigma^{\prime}}, \psi_{h_{1}, \sigma}\right\rangle\left\langle\psi_{h_{2}, \sigma^{\prime}}, \psi_{h_{2}, \sigma}\right\rangle+R\left(t, \sigma, \sigma^{\prime}\right) \text { with } \\
\left|R\left(t, \sigma, \sigma^{\prime}\right)\right| \leq & \frac{c}{\sigma^{\delta_{\lambda_{0}}}}\left(\frac{1}{t} \frac{1}{\sigma^{1 /\left(8 \gamma_{0}\right)}}+\left(\sigma^{\prime}\right)^{\bar{\alpha} /\left(4 \gamma_{0}\right)}\right) \\
\left\|\partial_{t} \Psi_{t, \sigma}\right\| \leq & \frac{c}{\sigma^{\delta_{\lambda_{0}}}}\left(\frac{\sigma^{\bar{\alpha} /\left(4 \gamma_{0}\right)}}{t}+\sigma t+\frac{1}{t^{2} \sigma^{1 /\left(4 \gamma_{0}\right)}}\right) \\
& +c \sigma^{1-\delta_{\lambda_{0}}}+c \sigma^{1 / 2-\delta_{\lambda_{0}}}\left(1+\frac{1}{t} \frac{1}{\sigma^{1 /\left(8 \gamma_{0}\right)}}\right) .
\end{aligned}
$$

Then one can choose $\lambda_{0}^{\prime} \in\left(0, \lambda_{0}\right]$ s.t. for $\lambda \in\left(0, \lambda_{0}^{\prime}\right]$ there exists the limit

$$
\Psi_{h_{1}, h_{2}}^{+}=\lim _{t \rightarrow \infty} \Psi_{t, \sigma_{t}},
$$

where $\sigma_{t}=\kappa / t^{\gamma}$. This limit is independent of the choice of $\gamma \in\left(4, \gamma_{0}\right]$.

Proof We assume that $t_{2} \geq t_{1} \geq 1$ are sufficiently large so that $\sigma_{t_{2}} \leq \sigma_{t_{1}} \leq 1$. We write

$$
\left\|\Psi_{t_{2}, \sigma_{t_{2}}}-\Psi_{t_{1}, \sigma_{t_{1}}}\right\| \leq\left\|\Psi_{t_{2}, \sigma_{t_{2}}}-\Psi_{t_{1}, \sigma_{t_{2}}}\right\|+\left\|\Psi_{t_{1}, \sigma_{t_{2}}}-\Psi_{t_{1}, \sigma_{t_{1}}}\right\| .
$$

Concerning the first term on the r.h.s. of (2.6), we note that the bound in (2.4) implies

$$
\left\|\partial_{t} \Psi_{t, \sigma_{t_{2}}}\right\| \leq \frac{c}{\sigma_{t_{2}}^{\delta_{\lambda_{0}}}}\left(\frac{\sigma_{t_{2}}^{\varepsilon}}{t}+\frac{1}{t^{2} \sigma_{t_{2}}^{1 /\left(4 \gamma_{0}\right)}}\right)+c \sigma_{t_{2}}^{1 / 2-\delta_{\lambda_{0}}} t
$$


for some $\varepsilon>0$, depending on $\gamma_{0}$, but independent of $\lambda_{0}$. Now we estimate

$$
\begin{aligned}
\left\|\Psi_{t_{2}, \sigma_{t_{2}}}-\Psi_{t_{1}, \sigma_{t_{2}}}\right\| & \leq \int_{t_{1}}^{t_{2}} d t\left\|\partial_{t} \Psi_{t, \sigma_{t_{2}}}\right\| \leq c \sigma_{t_{2}}^{\varepsilon-\delta_{\lambda_{0}}} \log \left(t_{2} / t_{1}\right)+c \frac{1}{t_{1}} \frac{1}{\sigma_{t_{2}}^{\delta_{\lambda_{0}}+1 /\left(4 \gamma_{0}\right)}}+c \sigma_{t_{2}}^{1 / 2-\delta_{\lambda_{0}}} t_{2}^{2} \\
& \leq c \sigma_{t_{2}}^{\varepsilon^{\prime}}+c \frac{1}{t_{1}} \frac{1}{\sigma_{t_{2}}^{1 /\left(3 \gamma_{0}\right)}}+c \sigma_{t_{2}}^{1 / 2-\delta_{\lambda_{0}}} t_{2}^{2} \leq c \frac{1}{t_{2}^{\varepsilon^{\prime \prime}}}+c \frac{t_{2}^{1 / 3}}{t_{1}} \\
& \leq c\left(\frac{t_{2}^{1 / 3}}{t_{1}}+\left(\frac{t_{2}^{1 / 3}}{t_{1}}\right)^{\varepsilon^{\prime \prime}}\right)
\end{aligned}
$$

where in the third step we made use of the fact that $t_{2}^{-\alpha} \log \left(t_{2} / t_{1}\right)$ is uniformly bounded in $t_{2} \geq t_{1} \geq 1$ for any $\alpha>0$ and chose $\lambda_{0}$ sufficiently small (depending on $\gamma_{0}$ ) to ensure that $\varepsilon^{\prime}>0$ and that $\delta_{\lambda_{0}}+1 /\left(4 \gamma_{0}\right) \leq 1 /\left(3 \gamma_{0}\right)$. In the fourth step we chose $\lambda_{0}$ sufficiently small and exploited the bound $\gamma>4$ to ensure that $1>\varepsilon^{\prime \prime}>0$.

As for the second term on the r.h.s. of (2.6), we get

$$
\begin{aligned}
\left\|\Psi_{t_{1}, \sigma_{t_{2}}}-\Psi_{t_{1}, \sigma_{t_{1}}}\right\|^{2} & =\left\langle\Psi_{t_{1}, \sigma_{t_{2}}}, \Psi_{t_{1}, \sigma_{t_{2}}}\right\rangle+\left\langle\Psi_{t_{1}, \sigma_{t_{1}}}, \Psi_{t_{1}, \sigma_{t_{1}}}\right\rangle-2 \operatorname{Re}\left\langle\Psi_{t_{1}, \sigma_{t_{2}}}, \Psi_{t_{1}, \sigma_{t_{1}}}\right\rangle \\
= & \left\langle\psi_{h_{1}, \sigma_{t_{2}}}, \psi_{h_{1}, \sigma_{t_{2}}}\right\rangle\left\langle\psi_{h_{2}, \sigma_{t_{2}}}, \psi_{h_{2}, \sigma_{t_{2}}}\right\rangle+R\left(t_{1}, \sigma_{t_{2}}, \sigma_{t_{2}}\right) \\
& +\left\langle\psi_{h_{1}, \sigma_{t_{1}}}, \psi_{h_{1}, \sigma_{t_{1}}}\right\rangle\left\langle\psi_{h_{2}, \sigma_{t_{1}}}, \psi_{h_{2}, \sigma_{t_{1}}}\right\rangle+R\left(t_{1}, \sigma_{t_{1}}, \sigma_{t_{1}}\right) \\
& -\left\langle\psi_{h_{1}, \sigma_{t_{2}}}, \psi_{h_{1}, \sigma_{t_{1}}}\right\rangle\left\langle\psi_{h_{2}, \sigma_{t_{2}}}, \psi_{h_{2}, \sigma_{t_{1}}}\right\rangle-\overline{R\left(t_{1}, \sigma_{t_{2}}, \sigma_{t_{1}}\right)} \\
& -\left\langle\psi_{h_{1}, \sigma_{t_{1}}}, \psi_{h_{1}, \sigma_{t_{2}}}\right\rangle\left\langle\psi_{h_{2}, \sigma_{t_{1}}}, \psi_{h_{2}, \sigma_{t_{2}}}\right\rangle-R\left(t_{1}, \sigma_{t_{2}}, \sigma_{t_{1}}\right) .
\end{aligned}
$$

Thus making use of Proposition 1.1(c) and (2.3), we obtain

$$
\begin{aligned}
\left\|\Psi_{t_{1}, \sigma_{t_{2}}}-\Psi_{t_{1}, \sigma_{t_{1}}}\right\|^{2} \leq & c \sigma_{t_{1}}^{\bar{\alpha}}+\left|R\left(t_{1}, \sigma_{t_{2}}, \sigma_{t_{2}}\right)\right|+\left|R\left(t_{1}, \sigma_{t_{1}}, \sigma_{t_{1}}\right)\right|+2\left|R\left(t_{1}, \sigma_{t_{2}}, \sigma_{t_{1}}\right)\right| \\
\leq & c \sigma_{t_{1}}^{\bar{\alpha}}+c \frac{1}{\sigma_{t_{2}}}\left(\frac{1}{\delta_{1}} \frac{1}{\sigma_{t_{2}}^{1 /\left(8 \gamma_{0}\right)}}+\left(\sigma_{t_{2}}\right)^{\bar{\alpha} /\left(4 \gamma_{0}\right)}\right) \\
& +c \frac{1}{\sigma_{t_{1}}^{\delta_{\lambda_{0}}}}\left(\frac{1}{t_{1}} \frac{1}{\sigma_{t_{1}}^{1 /\left(8 \gamma_{0}\right)}}+\left(\sigma_{t_{1}}\right)^{\bar{\alpha} /\left(4 \gamma_{0}\right)}\right) \\
& +c \frac{1}{\sigma_{t_{2}}^{\delta_{\lambda_{0}}}}\left(\frac{1}{t_{1}} \frac{1}{\sigma_{t_{2}}^{1 /\left(8 \gamma_{0}\right)}}+\left(\sigma_{t_{1}}\right)^{\bar{\alpha} /\left(4 \gamma_{0}\right)}\right)
\end{aligned}
$$

Thus, by choosing $\lambda_{0}$ sufficiently small, we can find $0<\delta_{1}, \delta_{2}, \delta<1$ s.t.

$$
\begin{aligned}
\left\|\Psi_{t_{1}, \sigma_{t_{2}}}-\Psi_{t_{1}, \sigma_{t_{1}}}\right\|^{2} & \leq c\left(\frac{1}{t_{1}^{\delta_{1}}}+\frac{t_{2}^{1 / 3}}{t_{1}}+\frac{1}{t_{2}^{\delta_{2}}}+\frac{t_{2}^{\delta_{\lambda_{0}}}}{t_{1}^{\delta_{1}}}\right) \\
& \leq c\left(\frac{t_{2}^{\delta_{\lambda_{0}}}}{t_{1}^{\delta}}+\frac{t_{2}^{1 / 3}}{t_{1}}\right) \leq c\left(\left(\frac{t_{2}^{1 / 3}}{t_{1}}\right)^{\delta}+\frac{t_{2}^{1 / 3}}{t_{1}}\right),
\end{aligned}
$$


where in the second step we used that $t_{2} \geq t_{1}$. Consequently

$$
\left\|\Psi_{t_{1}, \sigma_{t_{2}}}-\Psi_{t_{1}, \sigma_{t_{1}}}\right\| \leq c\left(\left(\frac{t_{2}^{1 / 3}}{t_{1}}\right)^{\delta / 2}+\left(\frac{t_{2}^{1 / 3}}{t_{1}}\right)^{1-\delta / 2}\right) .
$$

In view of (2.8), we get

$$
\left\|\Psi_{t_{2}, \sigma_{t_{2}}}-\Psi_{t_{1}, \sigma_{t_{1}}}\right\| \leq c \sum_{i=1}^{4}\left(\frac{t_{2}^{1 / 3}}{t_{1}}\right)^{\varepsilon_{i}}
$$

for $0<\varepsilon_{i} \leq 1$. Let us now set $\Psi(t):=\Psi_{t, \sigma_{t}}$ and proceed as in the proof of Theorem 3.1 of [25]: Suppose $t_{1}^{n} \leq t_{2}<t_{1}^{n+1}$. Then we can write

$$
\begin{aligned}
\left\|\Psi\left(t_{2}\right)-\Psi\left(t_{1}\right)\right\| & \leq\left(\sum_{k=1}^{n-1}\left\|\Psi\left(t_{1}^{k+1}\right)-\Psi\left(t_{1}^{k}\right)\right\|\right)+\left\|\Psi\left(t_{2}\right)-\Psi\left(t_{1}^{n}\right)\right\| \\
& \leq c \sum_{i=1}^{4} \sum_{k=1}^{n}\left(\frac{1}{t_{1}^{\varepsilon_{i}(2 k / 3-1 / 3)}}\right) \leq c \sum_{i=1}^{4} t_{1}^{-\varepsilon_{i} / 3} \frac{1}{1-\left(1 / t_{1}\right)^{2 \varepsilon_{i} / 3}} .
\end{aligned}
$$

Since the last expression tends to zero as $t_{1} \rightarrow \infty$, we obtain convergence of $t \mapsto \Psi(t)$ as $t \rightarrow \infty$.

Finally, let us show that the limit $\Psi_{h_{1}, h_{2}}^{+}$is independent of the choice of the parameter $\gamma \in\left(4, \gamma_{0}\right]$. Let $4<\gamma^{\prime} \leq \gamma$ and let $\sigma_{t}=\kappa / t^{\gamma}, \sigma_{t}^{\prime}=\kappa / t^{\gamma^{\prime}}$ so that $\sigma_{t} \leq \sigma_{t}^{\prime}$. We will show that

$$
\lim _{t \rightarrow \infty}\left\|\Psi_{t, \sigma_{t}}-\Psi_{t, \sigma_{t}^{\prime}}\right\|=0 \text {. }
$$

Similarly as in the first part of the proof, it follows from formula (2.2) and from Proposition 1.1(c) that

$$
\begin{aligned}
\lim _{t \rightarrow \infty}\left\langle\Psi_{t, \sigma_{t}}, \Psi_{t, \sigma_{t}}\right\rangle & =\lim _{t \rightarrow \infty}\left\langle\Psi_{t, \sigma_{t}^{\prime}}, \Psi_{t, \sigma_{t}}\right\rangle=\lim _{t \rightarrow \infty}\left\langle\Psi_{t, \sigma_{t}^{\prime}}, \Psi_{t, \sigma_{t}^{\prime}}\right\rangle \\
& =\left\langle\psi_{h_{1}}, \psi_{h_{1}}\right\rangle\left\langle\psi_{h_{2}}, \psi_{h_{2}}\right\rangle .
\end{aligned}
$$

This concludes the proof of (2.15).

In Proposition 2.2 below we derive a formula for $\partial_{t} \Psi_{t, \sigma}$, appearing in assumption (2.4). For the purpose of this derivation we introduce a suitable domain: First, we fix $l \in \mathbb{N}_{0}$, $r_{1}, r_{2}>0$ and consider vectors of the form

$$
\Psi_{l}^{r_{1}, r_{2}}=\sum_{m=0}^{\infty} \frac{1}{\sqrt{m !}} \int d^{3 l} p d^{3 m} k F_{l, m}(p ; k) \eta^{*}(p)^{l} a^{*}(k)^{m} \Omega,
$$

where $F_{l, m} \in L_{\text {sym }}^{2}\left(\mathcal{B}_{r_{1}}^{\times l} \times \mathcal{B}_{r_{2}}^{\times m}, d^{3 l} p d^{3 m} k\right)$, i.e., $F_{l, m}$ are square-integrable functions, symmetric (independently) in their electron and photon variables and supported in each electron (resp. photon) variable in a ball af radius $r_{1}$ (resp. $r_{2}$ ). Moreover, the norms of $F_{l, m}$ satisfy the bound

$$
\left\|F_{l, m}\right\|_{2} \leq \frac{c^{m}}{\sqrt{m !}}
$$


for some $c \geq 0$, independent of $m$, which guarantees that the vector (2.17) is well defined. Now we set

$$
\mathcal{D}:=\operatorname{Span}\left\{\Psi_{l}^{r_{1}, r_{2}} \mid l \in \mathbb{N}_{0}, r_{1}, r_{2}>0\right\},
$$

where Span means finite linear combinations. This domain is dense and it contains $\mathcal{C}$. We show in Lemma A.2 that $H_{\sigma}, H_{\mathrm{e}}, H_{\mathrm{f}}, H_{\mathrm{I}}^{\mathrm{a} / \mathrm{c}}$ and $\hat{\eta}^{*}(h), h \in C_{0}^{2}(S)$, are well-defined on $\mathcal{D}$ and leave this domain invariant.

Proposition 2.2 Let $h_{1}, h_{2} \in C_{0}^{2}(S)$ have disjoint supports and let $\Psi_{t, \sigma}$ be given by (2.1). Then there holds

$$
\begin{aligned}
\partial_{t} \Psi_{t, \sigma}= & e^{i t H}\left\{\frac{1}{2} i\left[\left[H_{\mathrm{I}}^{\mathrm{a}}, \hat{\eta}_{\sigma}^{*}\left(h_{1, t}\right)\right], \hat{\eta}_{\sigma}^{*}\left(h_{2, t}\right)\right] \Omega+i \hat{\eta}_{\sigma}^{*}\left(h_{1, t}\right) \check{H}_{\mathrm{I}, \sigma}^{\mathrm{c}} \hat{\eta}_{\sigma}^{*}\left(h_{2, t}\right) \Omega\right. \\
& \left.+i \hat{\eta}_{\sigma}^{*}\left(h_{1, t}\right) \hat{\eta}_{\sigma}^{*}\left(h_{2, t}^{\sigma}\right) \Omega\right\}+\{1 \leftrightarrow 2\} .
\end{aligned}
$$

The operators $H_{\mathrm{I}}^{\mathrm{a}}$ and $\check{H}_{I, \sigma}^{\mathrm{c}}$ are defined on $\mathcal{C}$ by

$$
\begin{aligned}
H_{\mathrm{I}}^{\mathrm{a}} & :=\int d^{3} p d^{3} k v_{\bar{\alpha}}(k) \eta^{*}(p+k) a(k) \eta(p), \\
\check{H}_{\mathrm{I}, \sigma}^{\mathrm{c}} & :=\int d^{3} p d^{3} k \check{v}_{\bar{\alpha}}^{\sigma}(k) \eta^{*}(p-k) a^{*}(k) \eta(p),
\end{aligned}
$$

where $\check{v}_{\bar{\alpha}}^{\sigma}(k):=\lambda \frac{\mathbf{1}_{\mathcal{B}_{\sigma}(k)|k|^{\bar{\alpha}}}}{(2|k|)^{1 / 2}}$ and $h_{i}^{\sigma}(p)=\left(E_{p, \sigma}-E_{p}\right) h_{i}(p)$. (Note that $\check{H}_{\mathrm{I}, \sigma}^{\mathrm{c}}$ involves only momenta below the infrared cut-off $\sigma$ ).

Proof We compute

$$
\begin{aligned}
\partial_{t} \Psi_{t, \sigma}= & e^{i H t} i H \hat{\eta}_{\sigma}^{*}\left(h_{1, t}\right) \hat{\eta}_{\sigma}^{*}\left(h_{2, t}\right) \Omega+e^{i H t} \hat{\eta}_{\sigma}^{*}\left(\partial_{t} h_{1, t}\right) \hat{\eta}_{\sigma}^{*}\left(h_{2, t}\right) \Omega+e^{i H t} \hat{\eta}_{\sigma}^{*}\left(h_{1, t}\right) \hat{\eta}_{\sigma}^{*}\left(\partial_{t} h_{2, t}\right) \Omega \\
= & e^{i H t} i H \hat{\eta}_{\sigma}^{*}\left(h_{1, t}\right) \hat{\eta}_{\sigma}^{*}\left(h_{2, t}\right) \Omega-e^{i H t} \hat{\eta}_{\sigma}^{*}\left(h_{2, t}\right) \hat{\eta}_{\sigma}^{*}\left(i\left(E h_{1}\right)_{t}\right) \Omega \\
& -e^{i H t} \hat{\eta}_{\sigma}^{*}\left(h_{1, t}\right) \hat{\eta}_{\sigma}^{*}\left(i\left(E h_{2}\right)_{t}\right) \Omega,
\end{aligned}
$$

where $\left(E h_{i}\right)(p):=E_{p} h_{i}(p), i=1,2$. The first term on the r.h.s. above is well defined by Lemma A.2. The equality

$$
\left(\partial_{t} \hat{\eta}_{\sigma}^{*}\left(h_{1, t}\right)\right) \hat{\eta}_{\sigma}^{*}\left(h_{2, t}\right) \Omega=\hat{\eta}_{\sigma}^{*}\left(\partial_{t} h_{1, t}\right) \hat{\eta}_{\sigma}^{*}\left(h_{2, t}\right) \Omega
$$

can easily be justified with the help of Lemmas B.4 and 3.3. Now we note the following identity, which is meaningful due to Lemma A.2:

$$
\begin{aligned}
i H \hat{\eta}_{\sigma}^{*}\left(h_{1, t}\right) \hat{\eta}_{\sigma}^{*}\left(h_{2, t}\right) \Omega= & i\left[\left[H, \hat{\eta}_{\sigma}^{*}\left(h_{1, t}\right)\right], \hat{\eta}_{\sigma}^{*}\left(h_{2, t}\right)\right] \Omega+\hat{\eta}_{\sigma}^{*}\left(h_{1, t}\right) i H \hat{\eta}_{\sigma}^{*}\left(h_{2, t}\right) \Omega \\
& +\hat{\eta}_{\sigma}^{*}\left(h_{2, t}\right) i H \hat{\eta}_{\sigma}^{*}\left(h_{1, t}\right) \Omega,
\end{aligned}
$$

where we made use of the fact that $H \Omega=0$. As for the first term on the r.h.s. of (2.25), we note that $\left[H_{\mathrm{fr}}, \hat{\eta}_{\sigma}^{*}\left(h_{1, t}\right)\right]$ and $\left[H_{\mathrm{I}}^{\mathrm{c}}, \hat{\eta}_{\sigma}^{*}\left(h_{1, t}\right)\right]$ are sums of products of creation operators and therefore commute with $\hat{\eta}_{\sigma}^{*}\left(h_{2, t}\right)$. Thus we get

$$
i\left[\left[H, \hat{\eta}_{\sigma}^{*}\left(h_{1, t}\right)\right], \hat{\eta}_{\sigma}^{*}\left(h_{2, t}\right)\right] \Omega=i\left[\left[H_{\mathrm{I}}^{\mathrm{a}}, \hat{\eta}_{\sigma}^{*}\left(h_{1, t}\right)\right], \hat{\eta}_{\sigma}^{*}\left(h_{2, t}\right)\right] \Omega .
$$


As for the second term on the r.h.s. of (2.25), we obtain

$$
\begin{aligned}
\hat{\eta}_{\sigma}^{*}\left(h_{1, t}\right) i H \hat{\eta}_{\sigma}^{*}\left(h_{2, t}\right) \Omega= & \hat{\eta}_{\sigma}^{*}\left(h_{1, t}\right) i\left(H-H_{\sigma}\right) \hat{\eta}_{\sigma}^{*}\left(h_{2, t}\right) \Omega+\hat{\eta}_{\sigma}^{*}\left(h_{1, t}\right) \hat{\eta}_{\sigma}^{*}\left(i\left(E_{\sigma} h_{2}\right)_{t}\right) \Omega \\
= & \hat{\eta}_{\sigma}^{*}\left(h_{1, t}\right) i \check{H}_{\mathrm{I}, \sigma}^{\mathrm{c}} \hat{\eta}_{\sigma}^{*}\left(h_{2, t}\right) \Omega+\hat{\eta}_{\sigma}^{*}\left(h_{1, t}\right) \hat{\eta}_{\sigma}^{*}\left(i\left(\left(E_{\sigma}-E\right) h_{2}\right)_{t}\right) \Omega \\
& +\hat{\eta}_{\sigma}^{*}\left(i h_{1, t}\right) \hat{\eta}_{\sigma}^{*}\left(\left(E h_{2}\right)_{t}\right) \Omega .
\end{aligned}
$$

Here in the first step we applied Lemma 2.3 and in the last step we made use of the fact that the operator

$$
\check{H}_{\mathrm{I}, \sigma}^{\mathrm{a}}:=\int d^{3} p d^{3} k \check{v}_{\bar{\alpha}}^{\sigma}(k) \eta^{*}(p+k) a(k) \eta(p)
$$

annihilates $\hat{\eta}_{\sigma}^{*}\left(h_{2, t}\right) \Omega$ due to the fact that $\check{v}_{\bar{\alpha}}^{\sigma}$ is supported below the infrared cut-off. As the last term on the r.h.s. of (2.25) can be treated analogously, this concludes the proof.

Lemma 2.3 Let $h \in C_{0}^{2}(S)$ and $\psi_{h, \sigma}:=\hat{\eta}_{\sigma}^{*}(h) \Omega$. Then the vector $\psi_{h, \sigma}$ equals

$$
\Pi^{*} \int^{\oplus} d p h(p) \psi_{p, \sigma} .
$$

Consequently, $H_{\sigma} \psi_{h, \sigma}=\psi_{E_{\sigma} h, \sigma}$, where $\left(E_{\sigma} h\right)(p):=E_{p, \sigma} h(p)$.

Proof With the electron's variables in the configuration space representation, the $m$-particle components of $\psi_{h, \sigma}$ have the form

$$
\begin{aligned}
\psi_{h, \sigma}^{m}\left(x, k_{1}, \ldots, k_{m}\right) & =\frac{1}{(2 \pi)^{3 / 2}} \int d^{3} p e^{i p x} h(p+\underline{k}) f_{p+\underline{k}, \sigma}^{m}\left(k_{1}, \ldots, k_{m}\right) \\
& =\frac{1}{(2 \pi)^{3 / 2}} e^{-i \underline{k} x} \int d^{3} p e^{i p x} h(p) f_{p, \sigma}^{m}\left(k_{1}, \ldots, k_{m}\right) .
\end{aligned}
$$

Consequently, the $m$-particle components of $\Pi\left(\psi_{h, \sigma}\right)$ are

$$
\begin{aligned}
\Pi\left(\psi_{h, \sigma}\right)^{m}\left(p^{\prime}, k_{1}, \ldots, k_{m}\right) & =\frac{1}{(2 \pi)^{3}} \int d^{3} x e^{-i p^{\prime} x} \int d^{3} p e^{i p x} h(p) f_{p, \sigma}^{m}\left(k_{1}, \ldots, k_{m}\right) \\
& =h\left(p^{\prime}\right) f_{p^{\prime}, \sigma}^{m}\left(k_{1}, \ldots, k_{m}\right)
\end{aligned}
$$

which concludes the proof.

\section{Vacuum Expectation Values of Renormalized Creation Operators}

In this section we estimate the norms of the terms appearing on the r.h.s. of (2.20) in order to verify assumption (2.4) in Theorem 2.1. We also derive estimates on the norms of scattering states which are required in Theorem 2.1 as well. A crucial input is provided by the nonstationary phase analysis in Sect. 4, which in turn relies on the spectral information from Theorem 1.2, proven in [12].

In this section we will use the following definitions:

$$
G_{i, m}(q ; k):=e^{-i E_{q} t} h_{i}(q) f_{q, \sigma}^{m}(k), \quad i \in\{1,2\},
$$


where $h_{1}, h_{2} \in C_{0}^{2}(S)$ and $f_{q, \sigma}^{m}(k)$ is defined in (1.55). (We stress that the functions $G_{i, m}$ are $t$-dependent, although this is not reflected by the notation). Moreover, we set

$$
B_{m}^{*}\left(G_{i, m}\right):=\int d^{3} q d^{3 m} k G_{i, m}(q ; k) a^{*}(k)^{m} \eta^{*}(q-\underline{k}) .
$$

Another convention, which we will use in this and the next section, concerns contractions of creation and annihilation operators. It is explained in full detail in Lemma B.2, so it is enough to illustrate it here by a simple example. Let us set $\tilde{n}=3, \tilde{m}=2, n=2, m=3$ and consider photon variables $\tilde{r} \in \mathbb{R}^{3 \tilde{n}}, \tilde{k} \in \mathbb{R}^{3 \tilde{m}}, r \in \mathbb{R}^{3 n}, k \in \mathbb{R}^{3 m}$. Then the expectation value

$$
\begin{aligned}
& \left\langle\Omega, a(\tilde{r})^{\tilde{n}} a(\tilde{k})^{\tilde{m}} a^{*}(r)^{n} a^{*}(k)^{m} \Omega\right\rangle \\
& \quad=\left\langle\Omega,\left\{a\left(\tilde{r}_{1}\right) a\left(\tilde{r}_{2}\right) a\left(\tilde{r}_{3}\right)\right\}\left\{a\left(\tilde{k}_{1}\right) a\left(\tilde{k}_{2}\right)\right\}\left\{a^{*}\left(r_{1}\right) a^{*}\left(r_{2}\right)\right\}\left\{a^{*}\left(k_{1}\right) a^{*}\left(k_{2}\right) a^{*}\left(k_{3}\right)\right\} \Omega\right\rangle
\end{aligned}
$$

is a sum of $(m+n)$ ! terms resulting from all the possible contraction patterns. Let us consider one of them:

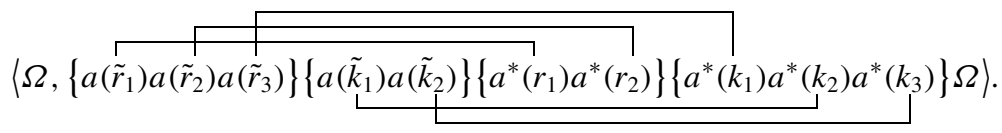

Given this contraction pattern, we make a decomposition $\tilde{r}=(\hat{\tilde{r}}, \check{\tilde{r}})$, where $\hat{\tilde{r}}=\left(\tilde{r}_{1}, \tilde{r}_{2}\right)$ are the $\tilde{r}$-variables which are contracted with some $r$-variables and $\tilde{\tilde{r}}=\tilde{r}_{3}$ is the $\tilde{r}$-variable contracted with a $k$-variable. In the case of the $\tilde{k}$-variables we have $\tilde{k}=\hat{\tilde{k}}$, since both $\tilde{k}_{1}$ and $\tilde{k}_{2}$ are contracted with $k$-variables. In this situation we say that $\check{\tilde{k}}$ is empty. Similarly, we have $r=\hat{r}$ with $\check{r}$ empty and $k=(\hat{k}, \check{k})$ with $\hat{k}=\left(k_{2}, k_{3}\right)$ and $\breve{k}=\left\{k_{1}\right\}$.

\subsection{Double Commutator}

It turns out that the behaviour of the double commutator on the r.h.s. of (2.20) is governed by the decay of the functions

$$
\begin{aligned}
& F_{n, m}^{G_{1}, G_{2}}(q ; r \mid p ; k) \\
& \quad:=(n+1) \int d^{3} r_{n+1} v_{\bar{\alpha}}\left(r_{n+1}\right) G_{1, n+1}\left(q+r_{n+1} ; r, r_{n+1}\right) G_{2, m}\left(p-r_{n+1} ; k\right),
\end{aligned}
$$

where $q, p \in \mathbb{R}^{3}, r \in \mathbb{R}^{3 n}, k \in \mathbb{R}^{3 m}$. For any such function we define an auxiliary operator

$$
\begin{aligned}
B_{n, m}^{*}\left(F_{n, m}^{G_{1}, G_{2}}\right):= & \int d^{3} q d^{3} p \int d^{3 n} r d^{3 m} k F_{n, m}^{G_{1}, G_{2}}(q ; r \mid p ; k) a^{*}(r)^{n} a^{*}(k)^{m} \\
& \times \eta^{*}(p-\underline{k}) \eta^{*}(q-\underline{r}) .
\end{aligned}
$$

Key properties of expectation values of such operators are given in Lemma B.3 below. Now we state and prove the estimate on the double commutator which gives rise to the first term on the r.h.s. of (2.4).

Proposition 3.1 There holds the bound

$$
\left\|\left[\left[H_{\mathrm{I}}^{\mathrm{a}}, \hat{\eta}_{\sigma}^{*}\left(h_{1, t}\right)\right], \hat{\eta}_{\sigma}^{*}\left(h_{2, t}\right)\right] \Omega\right\| \leq \frac{c}{\sigma^{\delta_{\lambda_{0}}}}\left(\frac{\sigma^{\bar{\alpha} /\left(4 \gamma_{0}\right)}}{t}+\sigma t+\frac{1}{t^{2} \sigma^{1 /\left(4 \gamma_{0}\right)}}\right) .
$$


Proof We compute

$$
\begin{aligned}
& \left\langle\left[\left[H_{\mathrm{I}}^{\mathrm{a}}, \hat{\eta}_{\sigma}^{*}\left(h_{1, t}\right)\right], \hat{\eta}_{\sigma}^{*}\left(h_{2, t}\right)\right] \Omega,\left[\left[H_{\mathrm{I}}^{\mathrm{a}}, \hat{\eta}_{\sigma}^{*}\left(h_{1, t}\right)\right], \hat{\eta}_{\sigma}^{*}\left(h_{2, t}\right)\right] \Omega\right\rangle \\
& =\sum_{\substack{m, n, \tilde{m}, \tilde{n} \in \mathbb{N}_{0} \\
m+n=\tilde{m}+\tilde{n}}} \frac{1}{\sqrt{m ! n ! \tilde{m} ! \tilde{n} !}} \\
& \quad \times\left\langle\left[\left[H_{\mathrm{I}}^{\mathrm{a}}, B_{\tilde{n}}^{*}\left(G_{1, \tilde{n}}\right)\right], B_{\tilde{m}}^{*}\left(G_{2, \tilde{m}}\right)\right] \Omega,\left[\left[H_{\mathrm{I}}^{\mathrm{a}}, B_{n}^{*}\left(G_{1, n}\right)\right], B_{m}^{*}\left(G_{2, m}\right)\right] \Omega\right\rangle, \\
& =\sum_{\substack{m, n, \tilde{m}, \tilde{n} \in \mathbb{N}_{0} \\
m+n=\tilde{m}+\tilde{n}}} \frac{1}{\sqrt{m ! n ! \tilde{m} ! \tilde{n} !}}\left\langle\left(B_{\tilde{n}-1, \tilde{m}}^{*}\left(F_{\tilde{n}-1, \tilde{m}}^{G_{1}, G_{2}}\right)+B_{\tilde{m}-1, \tilde{n}}^{*}\left(F_{\tilde{m}-1, \tilde{n}}^{G_{2}, G_{1}}\right)\right) \Omega,\right. \\
& \\
& \left.\quad\left(B_{n-1, m}^{*}\left(F_{n-1, m}^{G_{1}, G_{2}}\right)+B_{m-1, n}^{*}\left(F_{m-1, n}^{G_{2}, G_{1}}\right)\right) \Omega\right\rangle,
\end{aligned}
$$

where in the last step we made use of Lemma 3.2 and the operators $B_{m, n}^{*}(\cdot)$ are defined in (3.6). (By convention, $B_{-1, m}^{*}\left(F_{-1, m}^{G_{1}, G_{2}}\right) \Omega=B_{-1, n}^{*}\left(F_{-1, n}^{G_{2}, G_{1}}\right) \Omega=0$.) Given families of functions $G_{i_{1}, n}, G_{i_{2}, n}, G_{j_{1}, n}, G_{j_{2}, n}$ of the form (3.1), with $i_{1}, i_{2}, j_{1}, j_{2} \in\{1,2\}$, we define

$$
\begin{aligned}
& C\left(G_{i_{1}}, G_{i_{2}} ; G_{j_{1}}, G_{j_{2}}\right) \\
& \quad:=\sum_{\substack{m, n, \tilde{m}, \tilde{n} \in \mathbb{N}_{0} \\
m+n=\tilde{m}+\tilde{n}}} \frac{1}{\sqrt{m !(n+1) ! \tilde{m} !(\tilde{n}+1) !}}\left\langle B_{\tilde{n}, \tilde{m}}^{*}\left(F_{\tilde{n}, \tilde{m}}^{G_{i_{1}}, G_{i_{2}}}\right) \Omega, B_{n, m}^{*}\left(F_{n, m}^{G_{j_{1}}, G_{j_{2}}}\right) \Omega\right\rangle .
\end{aligned}
$$

From (3.8) we obtain

$$
\begin{aligned}
\left\|\left[\left[H_{\mathrm{I}}^{\mathrm{a}}, \hat{\eta}_{\sigma}^{*}\left(h_{1, t}\right)\right], \hat{\eta}_{\sigma}^{*}\left(h_{2, t}\right)\right] \Omega\right\|^{2}= & C\left(G_{1}, G_{2} ; G_{1}, G_{2}\right)+C\left(G_{2}, G_{1} ; G_{2}, G_{1}\right) \\
& +2 \operatorname{Re}\left(C\left(G_{1}, G_{2} ; G_{2}, G_{1}\right)\right) .
\end{aligned}
$$

In view of definition (3.1), $C\left(G_{2}, G_{1} ; G_{2}, G_{1}\right)$ can be obtained from $C\left(G_{1}, G_{2} ; G_{1}, G_{2}\right)$ by a substitution $\left(h_{1}, h_{2}\right) \rightarrow\left(h_{2}, h_{1}\right)$. Thus it suffices to consider $C\left(G_{1}^{\prime}, G_{2}^{\prime} ; G_{1}, G_{2}\right)$, where

$$
G_{i, m}^{\prime}(q ; k):=e^{-i E_{q} t} h_{i}^{\prime}(q) f_{q, \sigma}^{m}(k), \quad i \in\{1,2\}
$$

and $\left(h_{1}^{\prime}, h_{2}^{\prime}\right) \in\left\{\left(h_{1}, h_{2}\right),\left(h_{2}, h_{1}\right)\right\}$. We recall from Lemma B.3 that

$$
\begin{aligned}
& \left\langle B_{\tilde{n}, \tilde{m}}^{*}\left(F_{\tilde{n}, \tilde{m}}^{G_{1}^{\prime}, G_{2}^{\prime}}\right) \Omega, B_{n, m}^{*}\left(F_{n, m}^{G_{1}, G_{2}}\right) \Omega\right\rangle \\
& =\sum_{\rho \in S_{m+n}} \int d^{3} q d^{3} p \int d^{3 n} r d^{3 m} k F_{n, m}^{G_{1}, G_{2}}(q ; r \mid p ; k) \\
& \quad \times\left(\bar{F}_{\tilde{n}, \tilde{m}}^{G_{1}^{\prime}, G_{2}^{\prime}}(p-\underline{\hat{k}}+\underline{\hat{r}} ; \hat{r}, \check{k} \mid q+\underline{\hat{k}}-\underline{\hat{r}} ; \hat{k}, \check{r})\right. \\
& \left.\quad+\bar{F}_{\tilde{n}, \tilde{m}}^{G_{1}^{\prime}, G_{2}^{\prime}}(q+\underline{\underline{k}}-\underline{\check{r}} ; \hat{r}, \check{k} \mid p-\underline{\check{k}}+\underline{\underline{r}} ; \hat{k}, \check{r})\right) .
\end{aligned}
$$

The notation $\hat{k}, \breve{k}, \hat{r}, \check{r}$ is explained in Lemma B.2 and at the beginning of this section. Now from Lemma 4.1 we obtain

$$
\left|F_{n, m}^{G_{1}, G_{2}}(q ; r \mid p ; k)\right| \leq \frac{1}{\sigma^{\delta_{\lambda_{0}}}}\left(\frac{\sigma^{\bar{\alpha} /\left(4 \gamma_{0}\right)}}{t}+\sigma t+\frac{1}{t^{2} \sigma^{1 /\left(4 \gamma_{0}\right)}}\right) \frac{1}{\sqrt{m ! n !}} D(p, q) g_{\sigma}^{m}(k) g_{\sigma}^{n}(r),
$$


where $(p, q) \mapsto D(p, q)$ is a smooth, compactly supported function. From this bound we get

$$
\begin{aligned}
& \left|F_{n, m}^{G_{1}, G_{2}}(q ; r \mid p ; k) \bar{F}_{\tilde{n}, \tilde{m}}^{G_{1}^{\prime}, G_{2}^{\prime}}(p-\underline{\hat{k}}+\underline{\hat{r}} ; \hat{r}, \breve{k} \mid q+\underline{\hat{k}}-\underline{\hat{r}} ; \hat{k}, \check{r})\right| \\
& \quad \leq \frac{1}{\sigma^{2 \delta_{\lambda_{0}}}}\left(\frac{\sigma^{\bar{\alpha} /\left(4 \gamma_{0}\right)}}{t}+\sigma t+\frac{1}{t^{2} \sigma^{1 /\left(4 \gamma_{0}\right)}}\right)^{2} D^{\prime}(p, q) \frac{1}{\sqrt{m ! n ! \tilde{m} ! \tilde{n} !}} g_{\sigma}^{m}(k)^{2} g_{\sigma}^{n}(r)^{2}
\end{aligned}
$$

and similarly

$$
\begin{aligned}
& \left|F_{n, m}^{G_{1}, G_{2}}(q ; r \mid p ; k) \bar{F}_{\tilde{n}, \tilde{m}}^{G_{1}^{\prime}, G_{2}^{\prime}}(q+\underline{\underline{k}}-\underline{\check{r}} ; \hat{r}, \check{k} \mid p-\underline{\check{k}}+\underline{\check{r}} ; \hat{k}, \check{r})\right| \\
& \quad \leq \frac{1}{\sigma^{2 \delta_{\lambda_{0}}}}\left(\frac{\sigma^{\bar{\alpha} /\left(4 \gamma_{0}\right)}}{t}+\sigma t+\frac{1}{t^{2} \sigma^{1 /\left(4 \gamma_{0}\right)}}\right)^{2} D^{\prime}(p, q) \frac{1}{\sqrt{m ! n ! \tilde{m} ! \tilde{n} !}} g_{\sigma}^{m}(k)^{2} g_{\sigma}^{n}(r)^{2}
\end{aligned}
$$

where $D^{\prime}$ is again a smooth, compactly supported function. Making use of (3.9), (3.12) and the last two bounds we get

$$
\begin{aligned}
\left|C\left(G_{1}^{\prime}, G_{2}^{\prime} ; G_{1}, G_{2}\right)\right| & \leq \frac{1}{\sigma^{2 \delta_{\lambda_{0}}}}\left(\frac{\sigma^{\bar{\alpha} /\left(4 \gamma_{0}\right)}}{t}+\sigma t+\frac{1}{t^{2} \sigma^{1 /\left(4 \gamma_{0}\right)}}\right)^{2} \sum_{\substack{m, n, \tilde{m}, \tilde{n} \in \mathbb{N}_{0} \\
m+n=\tilde{m}+\tilde{n}}} \frac{(m+n) !}{\sqrt{m !(n+1) ! \tilde{m} !(\tilde{n}+1) !}} \\
& \times \frac{1}{\sqrt{m ! n ! \tilde{m} ! \tilde{n} !}}\left\|g_{\sigma}^{m}\right\|_{2}^{2}\left\|g_{\sigma}^{n}\right\|_{2}^{2} \\
\leq & \frac{1}{\sigma^{2 \delta_{\lambda_{0}}}}\left(\frac{\sigma^{\bar{\alpha} /\left(4 \gamma_{0}\right)}}{t}+\sigma t+\frac{1}{t^{2} \sigma^{1 /\left(4 \gamma_{0}\right)}}\right)^{2}\left(\frac{\kappa_{*}}{\sigma}\right)^{4 \lambda^{2} c^{2}} \\
\leq & \frac{c^{\prime}}{\sigma^{\delta_{\lambda_{0}}^{\prime}}}\left(\frac{\sigma^{\bar{\alpha} /\left(4 \gamma_{0}\right)}}{t}+\sigma t+\frac{1}{t^{2} \sigma^{1 /\left(4 \gamma_{0}\right)}}\right)^{2}
\end{aligned}
$$

where we made use of Lemma 3.3 and definition (1.48). This concludes the proof.

Lemma 3.2 For any $n, m \in \mathbb{N}_{0}$ there holds

$$
\left[\left[H_{\mathrm{I}}^{\mathrm{a}}, B_{n}^{*}\left(G_{1, n}\right)\right], B_{m}^{*}\left(G_{2, m}\right)\right] \Omega=B_{n-1, m}^{*}\left(F_{n-1, m}^{G_{1}, G_{2}}\right) \Omega+B_{m-1, n}^{*}\left(F_{m-1, n}^{G_{2}, G_{1}}\right) \Omega,
$$

where $F_{n-1, m}^{G_{1}, G_{2}}$ is defined in (3.5) and we set $B_{-1, m}^{*}\left(F_{-1, m}^{G_{1}, G_{2}}\right) \Omega=B_{-1, n}^{*}\left(F_{-1, n}^{G_{2}, G_{1}}\right) \Omega=0$.

Proof First we compute the inner commutator on $\mathcal{C}$ :

$$
\begin{aligned}
{\left[H_{\mathrm{I}}^{\mathrm{a}}, B_{n}^{*}\left(G_{1, n}\right)\right]=} & \int d^{3} q d^{3 n} r d^{3} u d^{3} w G_{1, n}(q ; r) v_{\bar{\alpha}}(w) \\
& \times\left[a(w) \eta^{*}(u+w) \eta(u), a^{*}(r)^{n} \eta^{*}(q-\underline{r})\right] .
\end{aligned}
$$


We note that

$$
\begin{aligned}
{\left[a(w) \eta^{*}(u+w) \eta(u), a^{*}(r)^{n} \eta^{*}(q-\underline{r})\right]=} & \sum_{i=1}^{n} \delta\left(w-r_{i}\right) a^{*}\left(r_{i_{*}}\right)^{n-1} \eta^{*}(u+w) \eta(u) \eta^{*}(q-\underline{r}) \\
& +a(w) a^{*}(r)^{n} \delta(u-q+\underline{r}) \eta^{*}(u+w),
\end{aligned}
$$

where $a^{*}\left(r_{i_{*}}\right)^{n-1}=a^{*}\left(r_{1}\right) \cdots a^{*}\left(r_{i-1}\right) a^{*}\left(r_{i+1}\right) \cdots a^{*}\left(r_{n}\right)$ for $n \geq 1$ and $a^{*}\left(r_{i_{*}}\right)^{n-1}=0$ for $n=0$. Since $G_{1, n}$ is symmetric in the photon variables, the contributions to (3.18) proportional to the first and the second term on the r.h.s. of (3.19) are

$$
\begin{aligned}
& {\left[H_{\mathrm{I}}^{\mathrm{a}}, B_{n}^{*}\left(G_{1, n}\right)\right]_{1}} \\
& \quad:=n \int d^{3} q d^{3(n-1)} r d^{3} r_{n} d^{3} u G_{1, n}\left(q ; r, r_{n}\right) v_{\bar{\alpha}}\left(r_{n}\right) a^{*}(r)^{n-1} \eta^{*}\left(u+r_{n}\right) \eta(u) \eta^{*}\left(q-\underline{r}-r_{n}\right),
\end{aligned}
$$

and

$$
\left[H_{\mathrm{I}}^{\mathrm{a}}, B_{n}^{*}\left(G_{1, n}\right)\right]_{2}:=\int d^{3} q d^{3 n} r d^{3} w G_{1, n}(q ; r) v_{\bar{\alpha}}(w) a(w) a^{*}(r)^{n} \eta^{*}(q-\underline{r}+w),
$$

respectively. Now let us compute the first contribution to the double commutator:

$$
\begin{aligned}
& {\left[\left[H_{\mathrm{I}}^{\mathrm{a}}, B_{n}^{*}\left(G_{1, n}\right)\right]_{1}, B_{m}^{*}\left(G_{2, m}\right)\right] \Omega} \\
& =n \int d^{3} q d^{3(n-1)} r d^{3} r_{n} d^{3} u \int d^{3} p d^{3 m} k G_{1, n}\left(q ; r, r_{n}\right) v_{\bar{\alpha}}\left(r_{n}\right) G_{2, m}(p ; k) \\
& \quad \times\left[a^{*}(r)^{n-1} \eta^{*}\left(u+r_{n}\right) \eta(u) \eta^{*}\left(q-\underline{r}-r_{n}\right), a^{*}(k)^{m} \eta^{*}(p-\underline{k})\right] \Omega \\
& =n \int d^{3} q d^{3} p \int d^{3(n-1)} r d^{3} r_{n} d^{3 m} k G_{1, n}\left(q ; r, r_{n}\right) v_{\bar{\alpha}}\left(r_{n}\right) G_{2, m}(p ; k) \\
& \quad \times a^{*}(r)^{n-1} a^{*}(k)^{m} \eta^{*}\left(p-\underline{k}+r_{n}\right) \eta^{*}\left(q-\underline{r}-r_{n}\right) \Omega .
\end{aligned}
$$

By changing variables $p \rightarrow p-r_{n}$ and $q \rightarrow q+r_{n}$, we get

$$
\left[\left[H_{\mathrm{I}}^{\mathrm{a}}, B_{n}^{*}\left(G_{1, n}\right)\right]_{1}, B_{m}^{*}\left(G_{2, m}\right)\right] \Omega=B_{n-1, m}^{*}\left(F_{n-1, m}^{G_{1}, G_{2}}\right) \Omega .
$$

The second contribution to the double commutator has the form:

$$
\begin{aligned}
& {\left[\left[H_{\mathrm{I}}^{\mathrm{a}}, B_{n}^{*}\left(G_{1, n}\right)\right]_{2}, B_{m}^{*}\left(G_{2, m}\right)\right] \Omega} \\
& =\int d^{3} q d^{3 n} r d^{3} w \int d^{3} p d^{3 m} k G_{1, n}(q ; r) v_{\bar{\alpha}}(w) G_{2, m}(p ; k) \\
& \quad \times\left[a(w) a^{*}(r)^{n} \eta^{*}(q-\underline{r}+w), a^{*}(k)^{m} \eta^{*}(p-\underline{k})\right] \Omega \\
& =m \int d^{3} q d^{3} p \int d^{3 n} r d^{3(m-1)} k d^{3} k_{m} G_{1, n}(q ; r) v_{\bar{\alpha}}\left(k_{m}\right) G_{2, m}\left(p ; k, k_{m}\right) \\
& \quad \times a^{*}(r)^{n} a^{*}(k)^{m-1} \eta^{*}\left(q-\underline{r}+k_{m}\right) \eta^{*}\left(p-\underline{k}-k_{m}\right) \Omega .
\end{aligned}
$$

By changing variables $q \rightarrow q-k_{m}$ and $p \rightarrow p+k_{m}$ we obtain

$$
\left[\left[H_{\mathrm{I}}^{\mathrm{a}}, B_{n}^{*}\left(G_{1, n}\right)\right]_{2}, B_{m}^{*}\left(G_{2, m}\right)\right] \Omega=B_{m-1, n}^{*}\left(F_{m-1, n}^{G_{2}, G_{1}}\right) \Omega,
$$

which concludes the proof. 
Lemma 3.3 There hold the estimates

$$
\begin{gathered}
\sum_{\substack{m, n, \tilde{m}, \tilde{n} \in \mathbb{N}_{0}, m+n=\tilde{m}+\tilde{n}}} \frac{(\tilde{m}+\tilde{n}) !}{m ! n ! \tilde{m} ! \tilde{n} !}\left\|g_{\sigma}^{m}\right\|_{2}^{2}\left\|g_{\sigma}^{n}\right\|_{2}^{2} \leq\left(\frac{\kappa_{*}}{\sigma}\right)^{4 \lambda^{2} c^{2}}, \\
\sum_{\substack{m, n, \tilde{m}, \tilde{n} \in \mathbb{N}_{0} \\
m+n=\tilde{m}+\tilde{n}}} \frac{(\tilde{m}+\tilde{n}) !}{m ! n ! \tilde{m} ! \tilde{n} !}\left\|g_{\sigma}^{m-1}\right\|_{2}^{2}\left\|g_{\sigma}^{n}\right\|_{2}^{2} \leq 2\left(\frac{\kappa_{*}}{\sigma}\right)^{4 \lambda^{2} c^{2}}, \\
\sum_{\substack{m, n, \tilde{m}, \tilde{n} \in \mathbb{N}_{0} \\
m+n=\tilde{m}+\tilde{n}}} \frac{(\tilde{m}+\tilde{n}) !}{m ! n ! \tilde{m} ! \tilde{n} !}\left\|g_{\sigma}^{m-1}\right\|_{2}^{2}\left\|g_{\sigma}^{n-1}\right\|_{2}^{2} \leq 4\left(\frac{\kappa_{*}}{\sigma}\right)^{4 \lambda^{2} c^{2}},
\end{gathered}
$$

where $g_{\sigma}^{m}, \kappa_{*}$ are defined in (1.48) and we set by convention $g_{\sigma}^{-1}=0$.

Proof By definition of the functions $g_{\sigma}^{m}$ in (1.48)

$$
\left\|g_{\sigma}^{m}\right\|_{2}^{2} \leq(\lambda c)^{2 m}\left(\log \left(\kappa_{*} / \sigma\right)\right)^{m} .
$$

Thus we get

$$
\begin{aligned}
& \sum_{\substack{m, n, \tilde{m}, \tilde{n} \in \mathbb{N}_{0} \\
m+n=\tilde{m}+\tilde{n}}} \frac{(\tilde{m}+\tilde{n}) !}{m ! n ! \tilde{m} ! \tilde{n} !}\left\|g_{\sigma}^{m}\right\|_{2}^{2}\left\|g_{\sigma}^{n}\right\|_{2}^{2} \\
& \leq \sum_{m, n \in \mathbb{N}_{0}}\left(\sum_{\substack{\tilde{m}, \tilde{n} \in \mathbb{N}_{0} \\
\tilde{m}+\tilde{n}=m+n}} \frac{(\tilde{m}+\tilde{n}) !}{\tilde{m} ! \tilde{n} !}\right) \frac{(\lambda c)^{2(m+n)}}{m ! n !}\left(\log \left(\kappa_{*} / \sigma\right)\right)^{m+n} \\
& \leq \sum_{m, n \in \mathbb{N}_{0}} \frac{(\sqrt{2} \lambda c)^{2(m+n)}}{m ! n !}\left(\log \left(\kappa_{*} / \sigma\right)\right)^{m+n}=\left(\frac{\kappa_{*}}{\sigma}\right)^{4 \lambda^{2} c^{2}} .
\end{aligned}
$$

Let us now prove (3.27):

$$
\begin{aligned}
& \sum_{\substack{m, n, \tilde{m}, \tilde{n} \in \mathbb{N}_{0} \\
m+n=\tilde{m}+\tilde{n}}} \frac{(\tilde{m}+\tilde{n}) !}{m ! n ! \tilde{m} ! \tilde{n} !}\left\|g_{\sigma}^{m-1}\right\|_{2}^{2}\left\|g_{\sigma}^{n}\right\|_{2}^{2} \\
& \leq \sum_{\substack{m, n, \tilde{m}, \tilde{n} \in \mathbb{N}_{0} \\
m+n+1=\tilde{m}+\tilde{n}}} \frac{(\tilde{m}+\tilde{n}) !}{m ! n ! \tilde{m} ! \tilde{n} !}\left\|g_{\sigma}^{m}\right\|_{2}^{2}\left\|g_{\sigma}^{n}\right\|_{2}^{2} \\
& \leq \sum_{m, n \in \mathbb{N}_{0}}\left(\sum_{\substack{\tilde{m}, \tilde{n} \in \mathbb{N}_{0} \\
m+\tilde{n}=m+n+1}} \frac{(\tilde{m}+\tilde{n}) !}{\tilde{m} ! \tilde{n} !}\right) \frac{(\lambda c)^{2(m+n)}}{m ! n !}\left(\log \left(\kappa_{*} / \sigma\right)\right)^{m+n} \\
& \leq 2 \sum_{m, n \in \mathbb{N}_{0}} \frac{(\sqrt{2} \lambda c)^{2(m+n)}}{m ! n !}\left(\log \left(\kappa_{*} / \sigma\right)\right)^{m+n}=2\left(\frac{\kappa_{*}}{\sigma}\right)^{4 \lambda^{2} c^{2}} .
\end{aligned}
$$

The bound (3.28) is proven analogously. This concludes the proof. 


\subsection{Clustering of Scalar Products}

In this subsection we consider clustering properties of scalar products of scattering states approximants. We will study the expression

$$
\left\langle\Psi_{t, \sigma^{\prime}}^{\prime}, \Psi_{t, \sigma}\right\rangle=\left\langle\Omega, \hat{\eta}_{\sigma^{\prime}}\left(h_{2, t}^{\prime}\right) \hat{\eta}_{\sigma^{\prime}}\left(h_{1, t}^{\prime}\right) \hat{\eta}_{\sigma}^{*}\left(h_{1, t}\right) \hat{\eta}_{\sigma}^{*}\left(h_{2, t}\right) \Omega\right\rangle .
$$

Recalling from (1.54) that the renormalized creation operators have the form

$$
\hat{\eta}_{\sigma}^{*}(h)=\sum_{m=0}^{\infty} \frac{1}{\sqrt{m !}} \int d^{3} p d^{3 m} k h(p) f_{p, \sigma}^{m}(k) a^{*}(k)^{m} \eta^{*}(p-\underline{k}),
$$

we obtain that (3.32) is a sum of integrals over vacuum expectation values of the electron and photon creation and annihilation operators. The contractions of the electron operators give rise to two terms

$$
\begin{aligned}
& \left\langle\Psi_{t, \sigma^{\prime}}^{\prime}, \Psi_{t, \sigma}\right\rangle^{(1)}:=\left\langle\Omega, \hat{\eta}_{\sigma^{\prime}}\left(h_{2, t}^{\left.\left.h_{2, t}\right) \hat{\eta}_{\sigma^{\prime}}\left(h_{1, t}^{\prime}\right) \hat{\eta}_{\sigma}^{*}\left(h_{1, t}\right) \hat{\eta}_{\sigma}^{*}\left(h_{2, t}\right) \Omega\right\rangle,}\right.\right. \\
& \left\langle\Psi_{t, \sigma^{\prime}}^{\prime}, \Psi_{t, \sigma}\right\rangle^{(2)}:=\left\langle\Omega, \hat{\eta}_{\sigma^{\prime}}(\overbrace{2, t}^{\prime}) \hat{\eta}_{\sigma^{\prime}}\left(h_{1, t}^{\prime}\right) \hat{\eta}_{\sigma}^{*}\left(h_{1, t}\right) \hat{\eta}_{\sigma}^{*}\left(h_{2, t}\right) \Omega\right\rangle,
\end{aligned}
$$

which we call 'direct' and 'exchange', respectively. We emphasize that the contractions in (3.34) and (3.35) above do not involve the photon creation and annihilation operators. The contractions of photon variables are the subject of the remaining part of this discussion.

As for the direct term, we distinguish two types of photon contraction patterns. The first type has the form

$$
\left\langle\Omega, a(\tilde{k})^{\tilde{m}} a(\tilde{r})^{\tilde{n}} a^{*}(r)^{n} a^{*}(k)^{m} \Omega\right\rangle,
$$

where the monomials of creation and annihilation operators above come from the respective renormalized creation operators in (3.34). By (3.36) we mean that all the $\tilde{k}$-variables are contracted with the $k$-variables and all the $\tilde{r}$-variables are contracted with the $r$-variables, and that $\tilde{m}=m, \tilde{n}=n$. (That is, $\check{k}$ and $\check{r}$ are empty in the terminology introduced below equation (3.4)). Clearly, for fixed $m, n$ there are $m ! n$ ! such contraction patterns and, as we will show in the proof of Proposition 3.4, after summation over $m, n$ they give rise to the first term on the r.h.s. of (3.38), which is $\left\langle\psi_{h_{1}^{\prime}, \sigma^{\prime}}, \psi_{h_{1}, \sigma}\right\rangle\left\langle\psi_{h_{2}^{\prime}, \sigma^{\prime}}, \psi_{h_{2}, \sigma}\right\rangle$. Contraction patterns of the second type are those for which $\breve{k}$ or $\breve{r}$ are non-empty. With the help of the non-stationary phase analysis from Lemma 4.2, it will be shown that the resulting terms contribute to the rest term $R\left(t, \sigma, \sigma^{\prime}\right)$ on the r.h.s. of (3.38), which eventually tends to zero as proven in Theorem 2.1.

As for the exchange term (3.35), we consider again two types of photon contraction patterns. Contractions of the first type have the form

$$
\left\langle\Omega, a(\tilde{k})^{\tilde{m}} a(\tilde{r})^{\tilde{n}} a^{*}(r)^{n} a^{*}(k)^{m} \Omega\right\rangle,
$$

i.e., $\hat{r}$ and $\hat{k}$ are empty. These contraction patterns give rise to the second term on the r.h.s. of (3.38), which is $\left\langle\psi_{h_{1}^{\prime}, \sigma^{\prime}}, \psi_{h_{2}, \sigma}\right\rangle\left\langle\psi_{h_{2}^{\prime}, \sigma^{\prime}}, \psi_{h_{1}, \sigma}\right\rangle$. Contraction patterns of the second type are 
those for which $\hat{r}$ or $\hat{k}$ are non-empty. The non-stationary phase analysis from Lemma 4.3 gives that these terms contribute to $R\left(t, \sigma, \sigma^{\prime}\right)$.

After this overview, we are ready to state and prove the main result of this subsection which yields estimate (2.3).

Proposition 3.4 Let $\kappa \geq \sigma^{\prime} \geq \sigma>0$ and let $\Psi_{t, \sigma}, \Psi_{t, \sigma^{\prime}}^{\prime}$ be two scattering states approximants given by (2.1) with momentum wave functions $h_{1}, h_{2}$ and $h_{1}^{\prime}, h_{2}^{\prime}$, respectively. (We recall that $\operatorname{supp} h_{1} \cap \operatorname{supp} h_{2}=\emptyset$ and $\operatorname{supp} h_{1}^{\prime} \cap \operatorname{supp} h_{2}^{\prime}=\emptyset$. However, $\operatorname{supp} h_{i} \cap \operatorname{supp} h_{j}^{\prime}$, $i, j \in\{1,2\}$ may be non-empty.) Then

$$
\begin{aligned}
\left\langle\Psi_{t, \sigma^{\prime}}^{\prime}, \Psi_{t, \sigma}\right\rangle= & \left\langle\psi_{h_{1}^{\prime}, \sigma^{\prime}}, \psi_{h_{1}, \sigma}\right\rangle\left\langle\psi_{h_{2}^{\prime}, \sigma^{\prime}}, \psi_{h_{2}, \sigma}\right\rangle \\
& +\left\langle\psi_{h_{1}^{\prime}, \sigma^{\prime}}, \psi_{h_{2}, \sigma}\right\rangle\left\langle\psi_{h_{2}^{\prime}, \sigma^{\prime}}, \psi_{h_{1}, \sigma}\right\rangle+R\left(t, \sigma, \sigma^{\prime}\right),
\end{aligned}
$$

where the rest term satisfies

$$
\left|R\left(t, \sigma, \sigma^{\prime}\right)\right| \leq C\left(h, h^{\prime}\right) \frac{1}{\sigma^{\delta_{\lambda_{0}}}}\left(\frac{1}{t} \frac{1}{\sigma^{1 /\left(8 \gamma_{0}\right)}}+\left(\sigma^{\prime}\right)^{\bar{\alpha} /\left(4 \gamma_{0}\right)}\right) .
$$

Here $C\left(h, h^{\prime}\right):=c\left\|h_{1}\right\|_{1}\left\|h_{2}\right\|_{1} \sum_{\beta_{1}, \beta_{2} ; 0 \leq\left|\beta_{1}\right|+\left|\beta_{2}\right| \leq 1}\left\|\partial^{\beta_{1}} h_{1}^{\prime}\right\|_{\infty}\left\|\partial^{\beta_{2}} h_{2}^{\prime}\right\|_{\infty}$ and the sum extends over multiindices $\beta_{1}, \beta_{2}$.

Proof Let us set

$$
\begin{aligned}
G_{i, m}(q ; k) & :=e^{-i E_{q} t} h_{i}(q) f_{q, \sigma}^{m}(k), \\
G_{i, m}^{\prime}(q ; k) & :=e^{-i E_{q} t} h_{i}^{\prime}(q) f_{q, \sigma^{\prime}}^{m}(k),
\end{aligned}
$$

for $i \in\{1,2\}$. Now we can write

$$
\left\langle\Psi_{t, \sigma^{\prime}}^{\prime}, \Psi_{t, \sigma}\right\rangle=\sum_{\substack{m, n, \tilde{m}, \tilde{n} \in \mathbb{N}_{0} \\ \tilde{m}+\tilde{n}=m+n}} \frac{1}{\sqrt{m ! n ! \tilde{m} ! \tilde{n} !}}\left\langle\Omega, B_{\tilde{n}}\left(G_{1, \tilde{n}}^{\prime}\right) B_{\tilde{m}}\left(G_{2, \tilde{m}}^{\prime}\right) B_{n}^{*}\left(G_{1, n}\right) B_{m}^{*}\left(G_{2, m}\right) \Omega\right\rangle
$$

Making use of Lemma B.4, we obtain

$$
\begin{aligned}
\langle\Omega, & \left.B_{\tilde{n}}\left(G_{1, \tilde{n}}^{\prime}\right) B_{\tilde{m}}\left(G_{2, \tilde{m}}^{\prime}\right) B_{n}^{*}\left(G_{1, n}\right) B_{m}^{*}\left(G_{2, m}\right) \Omega\right\rangle \\
= & \sum_{\rho \in S_{m+n}} \int d^{3} q d^{3} p \int d^{3 n} r d^{3 m} k G_{1, n}(q ; r) G_{2, m}(p ; k) \\
& \times\left(\bar{G}_{1, \tilde{n}}^{\prime}(q+\underline{\check{k}}-\underline{\check{r}} ; \hat{r}, \check{k}) \bar{G}_{2, \tilde{m}}^{\prime}(p-\underline{\underline{k}}+\underline{\check{r}} ; \hat{k}, \check{r})\right. \\
& \left.+\bar{G}_{1, \tilde{n}}^{\prime}(p-\underline{\hat{k}}+\underline{\hat{r}} ; \hat{r}, \check{k}) \bar{G}_{2, \tilde{m}}^{\prime}(q+\underline{\hat{k}}-\underline{\hat{r}} ; \hat{k}, \check{r})\right),
\end{aligned}
$$

where the notation in (3.43) is explained in Lemma B.2 and below (3.4).

Let us denote the summands involving the first term in the bracket on the r.h.s. of (3.43) by $I_{m, n, \tilde{m}, \tilde{n}}^{(1)}$. Let $\breve{I}_{m, n, \tilde{m}, \tilde{n}}^{(1)}$ be such summands coming from permutations for which $\breve{k}$ or $\check{r}$ are non-empty (cf. the discussion below formula (3.4)). We note that there are $(m+n)$ ! $m ! n$ ! such permutations. Let $\left\langle\Psi_{t, \sigma^{\prime}}^{\prime}, \Psi_{t, \sigma}\right\rangle^{(\check{1})}$ be the contribution to (3.42) involving all the 
summands $\check{I}_{m, n, \tilde{m}, \tilde{n}}^{(1)}$. Making use of Lemmas 4.2 and 3.3, we get

$$
\begin{aligned}
\left|\left\langle\Psi_{t, \sigma^{\prime}}^{\prime}, \Psi_{t, \sigma}\right\rangle^{(\check{1})}\right| & \leq C\left(h, h^{\prime}\right) \frac{1}{\sigma^{\delta_{\lambda_{0}}}}\left(\frac{1}{t} \frac{1}{\sigma^{1 /\left(8 \gamma_{0}\right)}}+\left(\sigma^{\prime}\right)^{\bar{\alpha} /\left(4 \gamma_{0}\right)}\right)\left(\frac{\kappa_{*}}{\sigma}\right)^{4 \lambda^{2} c^{2}} \\
& \leq C\left(h, h^{\prime}\right) \frac{1}{\sigma^{\delta_{\lambda_{0}}^{\prime}}}\left(\frac{1}{t} \frac{1}{\sigma^{1 /\left(8 \gamma_{0}\right)}}+\left(\sigma^{\prime}\right)^{\bar{\alpha} /\left(4 \gamma_{0}\right)}\right) .
\end{aligned}
$$

Clearly, this expression contributes to $R\left(t, \sigma, \sigma^{\prime}\right)$.

Now let $\hat{I}_{m, n, \tilde{m}, \tilde{n}}^{(1)}$ denote the expressions $I_{m, n, \tilde{m}, \tilde{n}}^{(1)}$ coming from permutations for which $\check{k}$ and $\check{r}$ are empty. (We note that there are $m ! n$ ! such permutations and that in this case $m=\tilde{m}$, $n=\tilde{n}$.) We obtain from Lemma B.1 that

$$
\begin{aligned}
\hat{I}_{m, n, \tilde{m}, \tilde{n}}^{(1)} & =\int d^{3} q d^{3 n} r G_{1, n}(q ; r) \bar{G}_{1, \tilde{n}}^{\prime}(q ; r) \int d^{3} p d^{3 m} k G_{2, m}(p ; k) \bar{G}_{2, \tilde{m}}^{\prime}(p ; k) \\
& =\frac{1}{n !} \frac{1}{m !}\left\langle\Omega, B_{\tilde{n}}\left(G_{1, \tilde{n}}^{\prime}\right) B_{n}^{*}\left(G_{1, n}\right) \Omega\right\rangle\left\langle\Omega, B_{\tilde{m}}\left(G_{2, \tilde{m}}^{\prime}\right) B_{m}^{*}\left(G_{2, m}\right) \Omega\right\rangle .
\end{aligned}
$$

Let $\left\langle\Psi_{t, \sigma^{\prime}}^{\prime}, \Psi_{t, \sigma}\right\rangle^{(\hat{1})}$ be the contribution to $\left\langle\Psi_{t, \sigma^{\prime}}^{\prime}, \Psi_{t, \sigma}\right\rangle$ involving all such $\hat{I}_{m, n, \tilde{m}, \tilde{n}}^{(1)}$. Since the sum over permutations in (3.43) gives the compensating factor $m$ ! $n$ !, we obtain

$$
\begin{aligned}
\left\langle\Psi_{t, \sigma^{\prime}}^{\prime}, \Psi_{t, \sigma}\right\rangle^{(\hat{1})} & =\sum_{m, n, \tilde{m}, \tilde{n}} \frac{1}{\sqrt{m ! n ! \tilde{m} ! \tilde{n} !}}\left\langle\Omega, B_{\tilde{n}}\left(G_{1, \tilde{n}}^{\prime}\right) B_{n}^{*}\left(G_{1, n}\right) \Omega\right\rangle\left\langle\Omega, B_{\tilde{m}}\left(G_{2, \tilde{m}}^{\prime}\right) B_{m}^{*}\left(G_{2, m}\right) \Omega\right\rangle \\
& =\left\langle\psi_{h_{1}^{\prime}, \sigma^{\prime}}, \psi_{h_{1}, \sigma}\right\rangle\left\langle\psi_{h_{2}^{\prime}, \sigma^{\prime}}, \psi_{h_{2}, \sigma}\right\rangle
\end{aligned}
$$

where in the last step we compared definition (3.2) of $B_{n}^{*}\left(G_{i, n}\right)$ with definition (1.54) of the renormalized creation operator and recalled that $\psi_{h_{i}, \sigma}=\hat{\eta}_{\sigma}^{*}\left(h_{i}\right) \Omega$.

The analysis of the summands involving the second term in bracket on the r.h.s. of (3.43) is analogous, so we can be brief: Let us denote such summands by $I_{m, n, \tilde{m}, \tilde{n}}^{(2)}$ and let $\hat{I}_{m, n, \tilde{m}, \tilde{n}}^{(2)}$ be the summands coming from permutations for which $\hat{k}$ or $\hat{r}$ are non-empty. We denote by $\left\langle\Psi_{t, \sigma^{\prime}}^{\prime}, \Psi_{t, \sigma}\right\rangle^{(\hat{2})}$ the contribution to (3.42) involving all $\hat{I}_{m, n, \tilde{m}, \tilde{n}}^{(2)}$. By Lemmas 4.3 and 3.3, we get

$$
\left|\left\langle\Psi_{t, \sigma^{\prime}}^{\prime}, \Psi_{t, \sigma}\right\rangle^{(\hat{2})}\right| \leq C\left(h, h^{\prime}\right) \frac{1}{\sigma^{\delta_{\lambda_{0}}^{\prime}}}\left(\frac{1}{t} \frac{1}{\sigma^{1 /\left(8 \gamma_{0}\right)}}+\left(\sigma^{\prime}\right)^{\bar{\alpha} /\left(4 \gamma_{0}\right)}\right) .
$$

This part contributes to the rest term.

Now let $\breve{I}_{m, n, \tilde{m}, \tilde{n}}^{(2)}$ be the expressions $I_{m, n, \tilde{m}, \tilde{n}}^{(2)}$ coming from permutations for which $\hat{k}$ and $\hat{r}$ are empty. Denoting the corresponding contribution to (3.42) by $\left\langle\Psi_{t, \sigma^{\prime}}^{\prime}, \Psi_{t, \sigma}\right\rangle^{(\check{2})}$, we get from Lemma B.1 that

$$
\left\langle\Psi_{t, \sigma^{\prime}}^{\prime}, \Psi_{t, \sigma}\right\rangle^{(\check{2})}=\left\langle\psi_{h_{1}^{\prime}, \sigma^{\prime}}, \psi_{h_{2}, \sigma}\right\rangle\left\langle\psi_{h_{2}^{\prime}, \sigma^{\prime}}, \psi_{h_{1}, \sigma}\right\rangle
$$

This concludes the proof.

The above proposition has a simple corollary which gives the last term on the r.h.s. of estimate (2.4). 
Corollary 3.5 Let $h_{1}, h_{2} \in C_{0}^{2}(S)$ have disjoint supports and let $h_{2}^{\sigma}(q)=\left(E_{q, \sigma}-E_{q}\right) h_{2}(q)$. Then

$$
\left\|\hat{\eta}_{\sigma}^{*}\left(h_{1, t}\right) \hat{\eta}_{\sigma}^{*}\left(h_{2, t}^{\sigma}\right) \Omega\right\| \leq c \sigma^{1 / 2-\delta_{\lambda_{0}}}\left(1+\frac{1}{t} \frac{1}{\sigma^{1 /\left(8 \gamma_{0}\right)}}\right) .
$$

Proof Making use of Proposition 3.4, we get

$$
\left\|\hat{\eta}_{\sigma}^{*}\left(h_{1, t}\right) \hat{\eta}_{\sigma}^{*}\left(h_{2, t}^{\sigma}\right) \Omega\right\|^{2}=\left\langle\psi_{h_{1}, \sigma}, \psi_{h_{1}, \sigma}\right\rangle\left\langle\psi_{h_{2}^{\sigma}, \sigma}, \psi_{h_{2}^{\sigma}, \sigma}\right\rangle+R(t, \sigma, \sigma)
$$

where $R(t, \sigma, \sigma)$ satisfies

$$
\begin{aligned}
|R(t, \sigma, \sigma)| & \leq C(h, h) \frac{1}{\sigma^{\delta_{\lambda_{0}}}}\left(\frac{1}{t} \frac{1}{\sigma^{1 /\left(8 \gamma_{0}\right)}}+(\sigma)^{\bar{\alpha} /\left(4 \gamma_{0}\right)}\right), \\
C(h, h) & =c\left\|h_{1}\right\|_{1}\left\|h_{2}^{\sigma}\right\|_{1} \sum_{\beta_{1}, \beta_{2} ; 0 \leq\left|\beta_{1}\right|+\left|\beta_{2}\right| \leq 1}\left\|\partial^{\beta_{1}} h_{1}\right\|_{\infty}\left\|\partial^{\beta_{2}} h_{2}^{\sigma}\right\|_{\infty} .
\end{aligned}
$$

Making use of (1.42) in Proposition 1.1, we obtain that $C(h, h) \leq c^{\prime} \sigma$ and therefore

$$
|R(t, \sigma, \sigma)| \leq c \sigma^{1-\delta_{\lambda_{0}}}\left(1+\frac{1}{t} \frac{1}{\sigma^{1 /\left(8 \gamma_{0}\right)}}\right) .
$$

Now making use of Lemma 2.3, we get $\left\|\psi_{h_{1}, \sigma}\right\|^{2}=\left\|h_{1}\right\|_{2}^{2}$ and $\left\|\psi_{h_{2}^{\sigma}, \sigma}\right\|^{2}=\left\|h_{2}^{\sigma}\right\|_{2}^{2}$. Exploiting (1.42) in Proposition 1.1 again, we get $\left\|\psi_{h_{2}^{\sigma}, \sigma}\right\|^{2} \leq c \sigma^{2}$. Consequently,

$$
\left\|\hat{\eta}_{\sigma}^{*}\left(h_{1, t}\right) \hat{\eta}_{\sigma}^{*}\left(h_{2, t}^{\sigma}\right) \Omega\right\|^{2} \leq c^{\prime} \sigma^{1-\delta_{\lambda_{0}}}\left(1+\frac{1}{t} \frac{1}{\sigma^{1 /\left(8 \gamma_{0}\right)}}\right) .
$$

This concludes the proof.

3.3 Contribution Involving $\check{H}_{\mathrm{I}, \sigma}^{\mathrm{c}}$

This subsection is devoted to the term involving $\check{H}_{\mathrm{I}, \sigma}^{\mathrm{c}}$ on the r.h.s. of (2.20). The following elementary proposition, which relies on Lemma B.5, gives the second term on the r.h.s. of (2.4).

Proposition 3.6 Let $\check{H}_{\mathrm{I}, \sigma}^{\mathrm{c}}$ be defined as in (2.22). Then there holds the bound

$$
\left\|\hat{\eta}_{\sigma}^{*}\left(h_{1, t}\right) \check{H}_{\mathrm{I}, \sigma}^{\mathrm{c}} \hat{\eta}_{\sigma}^{*}\left(h_{2, t}\right) \Omega\right\| \leq c \sigma^{1-\delta_{\lambda_{0}}}
$$

Proof We rewrite the expression from the statement of the proposition as follows:

$$
\begin{aligned}
& \left\langle\hat{\eta}_{\sigma}^{*}\left(h_{1, t}\right) \check{H}_{\mathrm{I}, \sigma}^{\mathrm{c}} \hat{\eta}_{\sigma}^{*}\left(h_{2, t}\right) \Omega, \hat{\eta}_{\sigma}^{*}\left(h_{1, t}\right) \check{H}_{\mathrm{I}, \sigma}^{\mathrm{c}} \hat{\eta}_{\sigma}^{*}\left(h_{2, t}\right) \Omega\right\rangle \\
& =\sum_{\substack{m, n, \tilde{m}, \tilde{n} \in \mathbb{N}_{0} \\
m+n=\tilde{m}+\tilde{n}}} \frac{1}{\sqrt{m ! n ! \tilde{m} ! \tilde{n} !}} \\
& \quad \times\left\langle\Omega, B_{\tilde{n}}\left(G_{2, \tilde{n}}\right)\left(\check{H}_{\mathrm{I}, \sigma}^{\mathrm{c}}\right)^{*} B_{\tilde{m}}\left(G_{1, \tilde{m}}\right) B_{n}^{*}\left(G_{1, n}\right) \check{H}_{\mathrm{I}, \sigma}^{\mathrm{c}} B_{m}^{*}\left(G_{2, m}\right) \Omega\right\rangle .
\end{aligned}
$$


Now Lemma B.5 gives

$$
\begin{aligned}
& \left\langle\Omega, B_{\tilde{n}}\left(G_{2, \tilde{n}}\right)\left(\check{H}_{\mathrm{I}, \sigma}^{\mathrm{c}}\right)^{*} B_{\tilde{m}}\left(G_{1, \tilde{m}}\right) B_{n}^{*}\left(G_{1, n}\right) \check{H}_{\mathrm{I}, \sigma}^{\mathrm{c}} B_{m}^{*}\left(G_{2, m}\right) \Omega\right\rangle \\
& =\sum_{\rho \in S_{m+n}} \int d^{3} q d^{3} p \int d^{3 n} r d^{3 m} k G_{1, n}(q ; r) G_{2, m}(p ; k) \\
& \quad \times\left(\int d^{3} \tilde{p} \check{v}_{\bar{\alpha}}^{\sigma}(\tilde{p})^{2} \bar{G}_{2, \tilde{n}}(p-\tilde{p}-\underline{\hat{k}}+\underline{\hat{r}} ; \hat{r}, \check{k}) \bar{G}_{1, \tilde{m}}(\tilde{p}+q+\underline{\hat{k}}-\underline{\hat{r}} ; \hat{k}, \check{r})\right. \\
& \left.\quad+\left\|\check{v}_{\bar{\alpha}}^{\sigma}\right\|_{2}^{2} \bar{G}_{2, \tilde{n}}(q+\underline{\underline{k}}-\underline{\underline{r}} ; \hat{r}, \check{k}) \bar{G}_{1, \tilde{m}}(p-\underline{\underline{k}}+\underline{\underline{r}} ; \hat{k}, \check{r})\right),
\end{aligned}
$$

where $S_{m+n}$ is the set of permutations of an $m+n$ element set. Making use of Theorem 1.2, and of definition (3.1), we obtain the bounds

$$
\begin{aligned}
& \left|G_{1, n}(q ; r) G_{2, m}(p ; k) \bar{G}_{2, \tilde{n}}(p-\tilde{p}-\underline{\hat{k}}+\underline{\hat{r}} ; \hat{r}, \breve{k}) \bar{G}_{1, \tilde{m}}(\tilde{p}+q+\underline{\hat{k}}-\underline{\hat{r}} ; \hat{k}, \check{r})\right| \\
& \quad \leq \frac{1}{\sqrt{m ! n ! \tilde{m} ! \tilde{n} !}} D(p, q) g_{\sigma}^{n}(r)^{2} g_{\sigma}^{m}(k)^{2}, \\
& \quad G_{1, n}(q ; r) G_{2, m}(p ; k) \bar{G}_{2, \tilde{n}}(q+\check{\check{k}}-\underline{\check{r}} ; \hat{r}, \check{k}) \bar{G}_{1, \tilde{m}}(p-\underline{\breve{k}}+\underline{\check{r}} ; \hat{k}, \check{r}) \mid \\
& \quad \leq \frac{1}{\sqrt{m ! n ! \tilde{m} ! \tilde{n} !}} D(p, q) g_{\sigma}^{n}(r)^{2} g_{\sigma}^{m}(k)^{2},
\end{aligned}
$$

where $(p, q) \mapsto D(p, q)$ is some smooth compactly supported function independent of $\sigma, t$. Consequently, the r.h.s. of (3.57) can be estimated by

$$
c\left\|\check{v}_{\bar{\alpha}}^{\sigma}\right\|_{2}^{2}(m+n) ! \frac{1}{\sqrt{m ! n ! \tilde{m} ! \tilde{n} !}}\left\|g_{\sigma}^{n}\right\|_{2}^{2}\left\|g_{\sigma}^{m}\right\|_{2}^{2} .
$$

Substituting this bound to (3.56) and making use of Lemma 3.3, we get

$$
\left\|\hat{\eta}_{\sigma}^{*}\left(h_{1, t}\right) \check{H}_{\mathrm{I}, \sigma}^{\mathrm{c}} \hat{\eta}_{\sigma}^{*}\left(h_{2, t}\right) \Omega\right\|^{2} \leq c\left\|\check{v}_{\bar{\alpha}}^{\sigma}\right\|_{2}^{2}\left(\frac{\kappa_{*}}{\sigma}\right)^{4 \lambda^{2} c^{2}} .
$$

Exploiting the fact that $\left\|\check{v}_{\bar{\alpha}}^{\sigma}\right\|_{2} \leq c \sigma$, we conclude the proof.

\section{Non-stationary Phase Arguments}

In this section we derive non-stationary phase estimates which entered into our analysis in Sects. 3.1 and 3.2. The spectral information from Proposition 1.1 and Theorem 1.2 is crucial for this part of our investigation.

Lemma 4.1 Let $G_{i, m}, i \in\{1,2\}$, be as specified in (3.1) and let $F_{n, m}^{G_{1}, G_{2}}$ be defined as in (3.5) i.e., it has the form

$$
\begin{aligned}
F_{n, m}^{G_{1}, G_{2}}(q ; r \mid p ; k)= & (n+1) \int d^{3} \tilde{r} v_{\bar{\alpha}}(\tilde{r}) e^{-i\left(E_{q+\tilde{r}}+E_{p-\tilde{r}}\right) t} h_{2}(p-\tilde{r}) h_{1}(q+\tilde{r}) \\
& \times f_{q+\tilde{r}, \sigma}^{n+1}(r, \tilde{r}) f_{p-\tilde{r}, \sigma}^{m}(k)
\end{aligned}
$$


where $h_{1}, h_{2} \in C_{0}^{2}(S)$ have disjoint supports. There holds the bound

$$
\left|F_{n, m}^{G_{1}, G_{2}}(q ; r \mid p ; k)\right| \leq \frac{1}{\sigma^{\delta_{\lambda_{0}}}}\left(\frac{\sigma^{\bar{\alpha} /\left(4 \gamma_{0}\right)}}{t}+\sigma t+\frac{1}{t^{2} \sigma^{1 /\left(4 \gamma_{0}\right)}}\right) \frac{1}{\sqrt{m ! n !}} D(p, q) g_{\sigma}^{m}(k) g_{\sigma}^{n}(r),
$$

where $D \in C_{0}^{\infty}\left(\mathbb{R}^{3} \times \mathbb{R}^{3}\right)$.

Proof For $\sigma<\kappa$, we introduce the slow cut-off $\sigma_{\mathrm{s}}:=\kappa(\sigma / \kappa)^{1 /\left(8 \gamma_{0}\right)}$, which clearly satisfies $\sigma<\sigma_{\mathrm{s}} \leq \kappa$. Let $\chi \in C^{\infty}\left(\mathbb{R}^{3}\right), 0 \leq \chi \leq 1$, be supported in $\mathcal{B}_{1+\varepsilon}$ (the ball of radius $1+\varepsilon$ ) for some $0<\varepsilon<1$ and be equal to one on $\mathcal{B}_{1}$. We set $\chi_{1}(\tilde{k}):=\chi\left(\tilde{k} / \sigma_{\mathrm{s}}\right), \chi_{2}(\tilde{k}):=1-\chi_{1}(\tilde{k})$ and define

$$
\begin{aligned}
F_{j, n, m}^{G_{1}, G_{2}}(q ; r \mid p ; k):= & \int d^{3} \tilde{r} v_{\bar{\alpha}}(\tilde{r}) \chi_{j}(\tilde{r}) e^{-i\left(E_{q+\tilde{r}}+E_{p-\tilde{r}}\right) t} h_{2}(p-\tilde{r}) h_{1}(q+\tilde{r}) \\
& \times f_{q+\tilde{r}, \sigma}^{n+1}(r, \tilde{r}) f_{p-\tilde{r}, \sigma}^{m}(k) .
\end{aligned}
$$

We set $V(q, p, \tilde{r}):=E_{q+\tilde{r}}+E_{p-\tilde{r}}$ and note that by disjointness of the velocity supports of $h_{1}, h_{2}$, the condition $h_{2}(p-\tilde{r}) h_{1}(q+\tilde{r}) \neq 0$, together with Proposition 1.1(a), implies that

$$
\left|\nabla_{\tilde{r}} V(q, p, \tilde{r})\right| \geq \varepsilon^{\prime}>0
$$

for some fixed $\varepsilon^{\prime}$. Thus we can write

$$
e^{-i V(q, p, \tilde{r}) t}=\frac{\nabla_{\tilde{r}} V(q, p, \tilde{r}) \cdot \nabla_{\tilde{r}} e^{-i V(q, p, \tilde{r}) t}}{(-i t)\left|\nabla_{\tilde{r}} V(q, p, \tilde{r})\right|^{2}} .
$$

Now we define the function

$$
J(q, p, \tilde{r}):=\frac{\nabla_{\tilde{r}} V(q, p, \tilde{r})}{\left|\nabla_{\tilde{r}} V(q, p, \tilde{r})\right|^{2}} h_{2}(p-\tilde{r}) h_{1}(q+\tilde{r}) \chi^{\kappa}(\tilde{r})
$$

where $\chi^{\kappa} \in C_{0}^{\infty}\left(\mathbb{R}^{3}\right)$ is equal to one on $\mathcal{B}_{\kappa}$ and vanishes outside of a slightly larger set. We note that, by Proposition 1.1(a), for any multiindex $\beta$ s.t. $0 \leq|\beta| \leq 1$

$$
\left|\partial_{\tilde{r}}^{\beta} J(q, p, \tilde{r})\right| \leq D(q, p)
$$

where $(q, p) \mapsto D(q, p)$ is a smooth, compactly supported function. Moreover, for $0 \leq$ $|\beta| \leq 2$

$$
\left|\partial_{\tilde{r}}^{\beta} v_{\bar{\alpha}}(\tilde{r})\right| \leq \frac{\chi_{3}(\tilde{r})|\tilde{r}|^{\bar{\alpha}}}{|\tilde{r}|^{\frac{1}{2}+|\beta|}}, \quad\left|\partial_{\tilde{r}}^{\beta} \chi_{j}(\tilde{r})\right| \leq \frac{c}{\left(\sigma_{\mathrm{s}}\right)^{|\beta|}},
$$

where $\chi_{3}$ is a smooth, compactly supported function, independent of $\sigma$. In addition, for $0 \leq|\beta| \leq 2$ we obtain from Theorem 1.2(c)

$$
\begin{aligned}
& \left|\partial_{\tilde{r}}^{\beta} f_{p-\tilde{r}, \sigma}^{m}(k)\right| \leq \frac{1}{\sqrt{m !}} \frac{c}{\sigma^{\delta_{\lambda_{0}}}} g_{\sigma}^{m}(k), \\
& \left|\partial_{\tilde{r}}^{\beta} f_{q+\tilde{r}, \sigma}^{n+1}(r, \tilde{r})\right| \leq \frac{1}{\sqrt{n !}} \frac{c}{\sigma^{\delta_{\lambda_{0}}}} \frac{|\tilde{r}|^{\bar{\alpha}}}{|\tilde{r}|^{3 / 2+|\beta|}} g_{\sigma}^{n}(r) .
\end{aligned}
$$


Now using the Gauss Law we obtain from (4.3)

$$
\begin{aligned}
& F_{1, n, m}^{G_{1}, G_{2}}(q ; r \mid p ; k) \\
& =\frac{1}{i t} \int_{\sigma \leq|\tilde{r}| \leq \sigma_{\mathrm{s}}} d^{3} \tilde{r} e^{-i V(q, p, \tilde{r}) t} \nabla_{\tilde{r}} \cdot\left(J(q, p, \tilde{r}) v_{\bar{\alpha}}(\tilde{r}) \chi_{1}(\tilde{r}) f_{q+\tilde{r}, \sigma}^{n+1}(r, \tilde{r}) f_{p-\tilde{r}, \sigma}^{m}(k)\right) \\
& \quad+\left.\frac{\sigma^{2}}{i t} \int d \Omega(\mathbf{n}) e^{-i V(q, p, \sigma \mathbf{n}) t} \mathbf{n} \cdot\left(J(q, p, \tilde{r}) v_{\bar{\alpha}}(\tilde{r}) \chi_{1}(\tilde{r}) f_{q+\tilde{r}, \sigma}^{n+1}(r, \tilde{r}) f_{p-\tilde{r}, \sigma}^{m}(k)\right)\right|_{\tilde{r}=\sigma \mathbf{n}},
\end{aligned}
$$

where $\mathbf{n}$ is the normal vector to the unit sphere and $d \Omega(\mathbf{n})$ is the spherical measure, that is

$$
\mathbf{n}=(\sin \theta \cos \varphi, \sin \theta \sin \varphi, \cos \theta)
$$

and $d \Omega(\mathbf{n})=\sin \theta d \theta d \varphi$ in the spherical coordinates $(\theta, \phi)$. (Notice that the contribution corresponding to the outer surface vanishes because of the smooth cut-off associated with $\left.\chi_{1}\right)$.

Let us consider the first term on the r.h.s. of (4.11). Let $I_{1}$ be the corresponding integrand. Making use of (4.7)-(4.10), we obtain

$$
\begin{aligned}
\left|I_{1}\right| & \leq \frac{1}{\sqrt{m ! n !}} \sum_{0 \leq\left|\beta_{1}\right|+\left|\beta_{2}\right|+\left|\beta_{3}\right| \leq 1} \frac{c}{\left(\sigma_{\mathrm{s}}\right)^{\left|\beta_{1}\right|}} \frac{D(q, p)}{\sigma^{\delta_{\lambda_{0}}}} \frac{\chi_{3}(\tilde{r})|\tilde{r}|^{2 \bar{\alpha}}}{|\tilde{r}|^{2+\left|\beta_{2}\right|+\left|\beta_{3}\right|}} g_{\sigma}^{n}(r) g_{\sigma}^{m}(k) \\
& \leq \frac{1}{\sqrt{m ! n !}} \frac{c D(q, p)}{\sigma^{\delta_{\lambda_{0}}}} \frac{\chi_{3}(\tilde{r})|\tilde{r}|^{2 \bar{\alpha}}}{|\tilde{r}|^{3}} g_{\sigma}^{n}(r) g_{\sigma}^{m}(k),
\end{aligned}
$$

where in the last step above we made use of the fact that $|\tilde{r}| \leq(1+\varepsilon) \sigma_{\mathrm{s}}$ in the region of integration. Now let $I_{2}$ be the integrand in the boundary integral on the r.h.s. of (4.11). Making use, again, of bounds (4.7)-(4.10), we get

$$
\begin{aligned}
\left|I_{2}\right| & \leq\left.\frac{1}{\sqrt{m ! n !}} \frac{c D(q, p)}{\sigma^{\delta_{\lambda_{0}}}} \frac{\chi_{3}(\tilde{r})|\tilde{r}|^{2 \bar{\alpha}}}{|\tilde{r}|^{2}} g_{\sigma}^{n}(r) g_{\sigma}^{m}(k)\right|_{\tilde{r}=\sigma \mathbf{n}} \\
& =\frac{1}{\sqrt{m ! n !}} \frac{c D(q, p)}{\sigma^{\delta_{\lambda_{0}}}} \frac{\chi_{3}(\sigma \mathbf{n}) \sigma^{2 \bar{\alpha}}}{\sigma^{2}} g_{\sigma}^{n}(r) g_{\sigma}^{m}(k) .
\end{aligned}
$$

Thus (4.11), (4.13), (4.14) give

$$
\left|F_{1, n, m}^{G_{1}, G_{2}}(q ; r \mid p ; k)\right| \leq \frac{1}{\sqrt{m ! n !}} \frac{c D(q, p)}{\sigma^{\delta_{\lambda_{0}}}} \frac{\left(\sigma_{\mathrm{s}}\right)^{2 \bar{\alpha}}}{t} g_{\sigma}^{n}(r) g_{\sigma}^{m}(k),
$$

which is the first contribution to the bound in (4.2).

Now we consider the contribution above the slow cut-off. In this region we will have to differentiate by parts twice, so a direct application of the non-stationary phase method would result in third derivatives of the function $V(q, p, \tilde{r}):=E_{q+\tilde{r}}+E_{p-\tilde{r}}$. However, our spectral results do not include the existence of third derivatives of $p \mapsto E_{p}$, but rather the bound

$$
\left|\partial_{p}^{\beta} E_{p, \sigma}\right| \leq \frac{c}{\sigma^{\delta_{\lambda_{0}}}}
$$


for any multiindex $\beta$ s.t. $0 \leq|\beta| \leq 3$ (cf. Proposition 1.1(a)). To be able to exploit this bound, we introduce an auxiliary function $V_{\sigma}(q, p, \tilde{r}):=E_{q+\tilde{r}, \sigma}+E_{p-\tilde{r}, \sigma}$ and rewrite $F_{2, n, m}^{G_{1}, G_{2}}(q ; r \mid p ; k)$, defined in (4.3), as follows

$$
\begin{aligned}
F_{2, n, m}^{G_{1}, G_{2}}(q ; r \mid p ; k)= & \int d^{3} \tilde{r} v_{\bar{\alpha}}(\tilde{r}) \chi_{2}(\tilde{r}) e^{-i V_{\sigma}(q, p, \tilde{r}) t} h_{2}(p-\tilde{r}) h_{1}(q+\tilde{r}) f_{q+\tilde{r}, \sigma}^{n+1}(r, \tilde{r}) f_{p-\tilde{r}, \sigma}^{m}(k) \\
& +R(\sigma t) .
\end{aligned}
$$

The rest term $R(t \sigma)$ satisfies

$$
\begin{aligned}
|R(t \sigma)| \leq & \int d^{3} \tilde{r} v_{\bar{\alpha}}(\tilde{r}) \chi_{2}(\tilde{r}) \mid\left(1-e^{i\left(V(q, p, \tilde{r})-V_{\sigma}(q, p, \tilde{r})\right) t}\right) h_{2}(p-\tilde{r}) h_{1}(q+\tilde{r}) \\
& \times f_{q+\tilde{r}, \sigma}^{n+1}(r, \tilde{r}) f_{p-\tilde{r}, \sigma}^{m}(k) \mid \\
\leq & c \frac{\sigma t}{\sigma^{\delta_{\lambda_{0}}}} g_{\sigma}^{m}(k) g_{\sigma}^{n}(r),
\end{aligned}
$$

where we made use of the fact that $\left|V(q, p, \tilde{r})-V_{\sigma}(q, p, \tilde{r})\right| \leq c \sigma$ (Proposition 1.1(a)) and of bounds (4.8), (4.9), (4.10). This gives the second contribution to the bound in (4.2).

Let us denote by $F_{2, \sigma, n, m}^{G_{1}, G_{2}}$ the first term on the r.h.s. of (4.17). We will estimate this term with the help of the method of non-stationary phase. Similarly as in the first part of the proof we note that by disjointness of velocity supports of $h_{1}, h_{2}$, the condition $h_{2}(p-\tilde{r}) \times$ $h_{1}(q+\tilde{r}) \neq 0$ implies that

$$
\left|\nabla_{\tilde{r}} V_{\sigma}(q, p, \tilde{r})\right| \geq \varepsilon^{\prime \prime}>0
$$

for some fixed $\varepsilon^{\prime \prime}$, independent of $\sigma \in(0, \kappa]$. (Here we made use of the fact that $S \ni p \mapsto$ $E_{p, \sigma}$ is strictly convex, uniformly in $\sigma$. Cf. Proposition 1.1(a)). Thus we can write

$$
e^{-i V_{\sigma}(q, p, \tilde{r}) t}=\frac{\nabla_{\tilde{r}} V_{\sigma}(q, p, \tilde{r}) \cdot \nabla_{\tilde{r}} e^{-i V_{\sigma}(q, p, \tilde{r}) t}}{(-i t)\left|\nabla_{\tilde{r}} V_{\sigma}(q, p, \tilde{r})\right|^{2}} .
$$

We define the function

$$
J_{\sigma}(q, p, \tilde{r}):=\frac{\nabla_{\tilde{r}} V_{\sigma}(q, p, \tilde{r})}{\left|\nabla_{\tilde{r}} V_{\sigma}(q, p, \tilde{r})\right|^{2}} h_{2}(p-\tilde{r}) h_{1}(q+\tilde{r}) \chi^{\kappa}(\tilde{r}),
$$

which is analogous to the function $J$ introduced in (4.6) above. Making use of (4.16) and (4.19), we get for all multiindices $\beta$ s.t. $0 \leq|\beta| \leq 2$

$$
\left|\partial_{\tilde{r}}^{\beta} J_{\sigma}(q, p, \tilde{r})\right| \leq \frac{D(q, p)}{\sigma^{\delta_{\lambda_{0}}}} .
$$

By integrating twice by parts in the defining expression for $F_{2, \sigma, n, m}^{G_{1}, G_{2}}$, we get

$$
\begin{aligned}
F_{2, \sigma, n, m}^{G_{1}, G_{2}}(q ; r \mid p ; k)= & \frac{1}{(i t)^{2}} \int d^{3} \tilde{r} e^{-i V_{\sigma}(q, p, \tilde{r}) t} \\
& \times \nabla_{\tilde{r}} \cdot\left(\frac{\nabla_{\tilde{r}} V_{\sigma}(q, p, \tilde{r})}{\left|\nabla_{\tilde{r}} V_{\sigma}(q, p, \tilde{r})\right|^{2}}\right. \\
& \left.\times \nabla_{\tilde{r}} \cdot\left(J_{\sigma}(q, p, \tilde{r}) v_{\bar{\alpha}}(\tilde{r}) \chi_{2}(\tilde{r}) f_{q+\tilde{r}, \sigma}^{n+1}(r, \tilde{r}) f_{p-\tilde{r}, \sigma}^{m}(k)\right)\right) .
\end{aligned}
$$


In view of (4.16), (4.19), the function

$$
(q, p, \tilde{r}) \mapsto \frac{\nabla_{\tilde{r}} V_{\sigma}(q, p, \tilde{r})}{\left|\nabla_{\tilde{r}} V_{\sigma}(q, p, \tilde{r})\right|^{2}}
$$

is bounded by $c / \sigma^{\delta_{\lambda_{0}}}$, together with its first derivatives, on the support of $(q, p, \tilde{r}) \mapsto h_{2}(p-$ $\tilde{r}) h_{1}(q+\tilde{r}) \chi^{\kappa}(\tilde{r})$. Thus we obtain from the bounds (4.8), (4.9), (4.10) and (4.22) that the integrand $I$ in (4.23) satisfies

$$
\begin{aligned}
|I| & \leq \frac{1}{\sqrt{m ! n !}} \sum_{0 \leq\left|\beta_{1}\right|+\left|\beta_{2}\right|+\left|\beta_{3}\right| \leq 2} \frac{c}{\left(\sigma_{\mathrm{s}}\right)^{\left|\beta_{1}\right|}} \frac{D(q, p)}{\sigma^{\delta_{\lambda_{0}}}} \frac{\chi_{3}(\tilde{r})|\tilde{r}|^{2 \bar{\alpha}}}{|\tilde{r}|^{2+\left|\beta_{2}\right|+\left|\beta_{3}\right|}} g_{\sigma}^{n}(r) g_{\sigma}^{m}(k) \\
& \leq \frac{1}{\sqrt{m ! n !}} \frac{c}{\left(\sigma_{\mathrm{s}}\right)^{2}} \frac{D(q, p)}{\sigma^{\delta_{\lambda_{0}}}} \frac{\chi_{3}(\tilde{r})|\tilde{r}|^{2 \bar{\alpha}}}{|\tilde{r}|^{2}} g_{\sigma}^{n}(r) g_{\sigma}^{m}(k),
\end{aligned}
$$

where in the second step we made use of the fact that $\sigma_{\mathrm{s}} \leq|\tilde{r}|$ in the region of integration. Thus we get from (4.23) that

$$
\left|F_{2, \sigma, n, m}^{G_{1}, G_{2}}(q ; r \mid p ; k)\right| \leq \frac{1}{\sqrt{m ! n !}} \frac{c D(q, p)}{\sigma^{\delta_{\lambda_{0}}}} \frac{1}{t^{2}\left(\sigma_{\mathrm{s}}\right)^{2}} g_{\sigma}^{n}(r) g_{\sigma}^{m}(k),
$$

which gives the third contribution to (4.2). The factor $(n+1)$, appearing in $(4.1)$, can be estimated by $2^{n}$ and incorporated into the constant appearing in the definition of $g_{\sigma}^{n}$.

Lemma 4.2 Let $\breve{I}_{m, n, \tilde{m}, \tilde{n}}^{(1)}$ be defined as follows

$$
\begin{aligned}
\check{I}_{m, n, \tilde{m}, \tilde{n}}^{(1)}:= & \int d^{3} q d^{3} p \int d^{3 n} r d^{3 m} k G_{1, n}(q ; r) G_{2, m}(p ; k) \\
& \times \bar{G}_{1, \tilde{n}}^{\prime}(q+\check{\underline{k}}-\underline{\check{r}} ; \hat{r}, \check{k}) \bar{G}_{2, \tilde{m}}^{\prime}(p-\underline{\underline{k}}+\underline{\check{r}} ; \hat{k}, \check{r}),
\end{aligned}
$$

where $G_{i, n}, G_{i, \tilde{n}}^{\prime}, i \in\{1,2\}$, appeared in (3.40), (3.41), the notation $k=(\hat{k}, \check{k}), r=(\hat{r}, \check{r})$ is explained in Lemma B.2 and we consider a permutation in (3.43) for which $\check{k}$ or $\check{r}$ are non-empty. Then there holds

$$
\begin{aligned}
\left|\check{I}_{m, n, \tilde{m}, \tilde{n}}^{(1)}\right| \leq & \left(\frac{1}{t}\left(\frac{1}{\sigma^{\delta_{\lambda_{0}}}}+\frac{1}{\sigma^{1 /\left(8 \gamma_{0}\right)}}\right)+\left(\sigma^{\prime}\right)^{\bar{\alpha} /\left(4 \gamma_{0}\right)}\right) \frac{C\left(h, h^{\prime}\right)}{\sqrt{m ! n ! \tilde{m} ! \tilde{n} !}} \\
& \times\left(\left\|g_{\sigma}^{n-1}\right\|_{2}^{2}+\left\|g_{\sigma}^{n}\right\|_{2}^{2}\right)\left(\left\|g_{\sigma}^{m-1}\right\|_{2}^{2}+\left\|g_{\sigma}^{m}\right\|_{2}^{2}\right),
\end{aligned}
$$

where $C\left(h, h^{\prime}\right):=c\left\|h_{1}\right\|_{1}\left\|h_{2}\right\|_{1} \sum_{\beta_{1}, \beta_{2} ; 0 \leq\left|\beta_{1}\right|+\left|\beta_{2}\right| \leq 1}\left\|\partial^{\beta_{1}} h_{1}^{\prime}\right\|_{\infty}\left\|\partial^{\beta_{2}} h_{2}^{\prime}\right\|_{\infty}$ and the sum extends over multiindices $\beta_{1}, \beta_{2}$. We note that for $\breve{k}$ (resp. $\left.\check{r}\right)$ non-empty we have $m \neq 0$ (resp. $\left.n \neq 0\right)$ and there always holds $m+n=\tilde{m}+\tilde{n}$. We set by convention $g_{\sigma}^{-1}=0$.

Proof By inserting the definitions of $G_{i, n}, G_{i, \tilde{n}}^{\prime}, i \in\{1,2\}$, we obtain

$$
\begin{aligned}
& \check{I}_{m, n, \tilde{m}, \tilde{n}}^{(1)}=\int d^{3} q d^{3} p \int d^{3 n} r d^{3 m} k e^{i\left(E_{p-\underline{\underline{k}}+\underline{\underline{r}}}-E_{q}\right) t} e^{i\left(E_{q+\underline{\underline{k}}-\underline{\underline{r}}}-E_{p}\right) t} h_{2}(p) h_{1}(q) \\
& \times \bar{h}_{2}^{\prime}(p-\underline{\check{k}}+\underline{\check{r}}) \bar{h}_{1}^{\prime}(q+\underline{\underline{k}}-\underline{\underline{r}}) f_{q, \sigma}^{n}(r) f_{p, \sigma}^{m}(k) \bar{f}_{q+\underline{\tilde{k}}-\underline{\underline{r}}, \sigma^{\prime}}(\hat{r}, \check{k}) \bar{f}_{p-\underline{\tilde{m}}+\underline{\underline{r}}, \sigma^{\prime}}^{\tilde{k}}(\hat{r}, \check{r}) .
\end{aligned}
$$


Since the expression on the r.h.s. of (4.29) and the bound (4.28) are invariant under the substitutions $k \leftrightarrow r, p \leftrightarrow q, h_{1} \leftrightarrow h_{2}, h_{1}^{\prime} \leftrightarrow h_{2}^{\prime}, m \leftrightarrow n, \tilde{m} \leftrightarrow \tilde{n}$ it suffices to consider the case of non-empty $\check{k}$. Similarly to the proof of Lemma 4.1, we introduce a slow infrared cutoff. Let us set $\sigma_{\mathrm{s}}^{\prime}:=\kappa\left(\sigma^{\prime} / \kappa\right)^{1 /\left(8 \gamma_{0}\right)}$, which clearly satisfies $\sigma^{\prime}<\sigma_{\mathrm{s}}^{\prime} \leq \kappa$ because $\sigma^{\prime}<\kappa$. Let $\chi \in C^{\infty}\left(\mathbb{R}^{3}\right), 0 \leq \chi \leq 1$, be supported in $\mathcal{B}_{1+\varepsilon}$ (the ball of radius $1+\varepsilon$ ) for some $0<\varepsilon<1$ and be equal to one on $\mathcal{B}_{1}$. We set $\chi_{1}\left(\breve{k}_{1}\right):=\chi\left(\breve{k}_{1} / \sigma_{\mathrm{s}}^{\prime}\right), \chi_{2}\left(\breve{k}_{1}\right):=1-\chi_{1}\left(\breve{k}_{1}\right)$, where $\breve{k}_{1}$ is the first component of $\check{k}$, and write for $j \in\{1,2\}$

$$
\begin{aligned}
& \check{I}_{m, n, \tilde{m}, \tilde{n}}^{(1)(j)}:=\int d^{3} q d^{3} p \int d^{3 n} r d^{3 m} k e^{i\left(E_{p-\underline{\underline{k}}+\underline{\underline{r}}}-E_{q}\right) t} e^{i\left(E_{q+\underline{\underline{k}}-\underline{r}}-E_{p}\right) t} \\
& \times h_{2}(p) h_{1}(q) \bar{h}_{2}^{\prime}(p-\underline{\check{k}}+\underline{\check{r}}) \bar{h}_{1}^{\prime}(q+\underline{\check{k}}-\underline{\check{r}}) f_{q, \sigma}^{n}(r) f_{p, \sigma}^{m}(k) \\
& \times\left(\chi_{j}\left(\check{k}_{1}\right) \bar{f}_{q+\underline{\tilde{n}}-\underline{\underline{r}}, \sigma^{\prime}}^{\tilde{r}}(\hat{r}, \check{k})\right) \bar{f}_{p-\underline{\tilde{m}}+\underline{\underline{m}}, \sigma^{\prime}}^{\tilde{k}}(\hat{k}, \check{r}) .
\end{aligned}
$$

Let us first consider (4.30) with $j=1$. We conclude from Theorem 1.2 and the definition of the functions $g_{\sigma}^{n}$ that

$$
\begin{aligned}
& \left|\chi_{1}\left(\check{k}_{1}\right) f_{q, \sigma}^{n}(r) f_{p, \sigma}^{m}(k) f_{q+\underline{\tilde{k}}-\underline{\underline{r}, \sigma^{\prime}}}^{\tilde{n}}(\hat{r}, \check{k}) f_{p-\underline{m}+\underline{\underline{m}}, \sigma^{\prime}}^{\tilde{m}}(\hat{k}, \check{r})\right| \\
& \quad \leq \frac{1}{\sqrt{m ! n ! \tilde{m} ! \tilde{n} !}} \chi_{1}\left(\check{k}_{1}\right) g_{\sigma}^{n}(r) g_{\sigma}^{m}(k) g_{\sigma^{\prime}}^{\tilde{n}}(\hat{r}, \check{k}) g_{\sigma^{\prime}}^{\tilde{m}}(\hat{k}, \check{r}) \\
& \quad \leq \frac{1}{\sqrt{m ! n ! \tilde{m} ! \tilde{n} !}} \frac{c \chi\left(\check{k}_{1} / \sigma_{\mathrm{s}}^{\prime}\right) \chi_{\left[\sigma^{\prime}, \kappa_{*}\right)}\left(\check{k}_{1}\right)^{2}\left|\check{k}_{1}\right|^{2 \bar{\alpha}}}{\left|\check{k}_{1}\right|^{3}} g_{\sigma}^{n}(r) g_{\sigma}^{m-1}\left(k^{\prime}\right) g_{\sigma^{\prime}}^{\tilde{n}-1}\left(\hat{r}, \check{k}^{\prime}\right) g_{\sigma^{\prime}}^{\tilde{m}}(\hat{k}, \check{r}) \\
& \quad \leq \frac{1}{\sqrt{m ! n ! \tilde{m} ! \tilde{n} !}} \frac{c \chi\left(\check{k}_{1} / \sigma_{\mathrm{s}}^{\prime}\right) \chi_{\left[\sigma^{\prime}, \kappa_{*}\right)}\left(\check{k}_{1}\right)^{2}\left|\check{k}_{1}\right|^{2 \bar{\alpha}}}{\left|\check{k}_{1}\right|^{3}} g_{\sigma}^{n}(r)^{2} g_{\sigma}^{m-1}\left(k^{\prime}\right)^{2},
\end{aligned}
$$

where we decomposed $k=\left(\check{k}_{1}, k^{\prime}\right), \check{k}=\left(\check{k}_{1}, \check{k}^{\prime}\right)$ and in the last step we made use of the fact that $g_{\sigma^{\prime}}^{\tilde{m}}(\hat{k}, \check{r}) \leq g_{\sigma}^{\tilde{m}}(\hat{k}, \check{r})$ and $g_{\sigma^{\prime}}^{\tilde{n}-1}\left(\hat{r}, \breve{k}^{\prime}\right) \leq g_{\sigma}^{\tilde{n}-1}\left(\hat{r}, \breve{k}^{\prime}\right)$ for $\sigma^{\prime} \geq \sigma$. Substituting (4.31) to (4.30), we get

$$
\left|\check{I}_{m, n, \tilde{m}, \tilde{n}}^{(1)(1)}\right| \leq\left(\sigma_{\mathrm{s}}^{\prime}\right)^{2 \bar{\alpha}} \frac{c\left\|h_{1}\right\|_{1}\left\|h_{2}\right\|_{1}\left\|h_{1}^{\prime}\right\|_{\infty}\left\|h_{2}^{\prime}\right\|_{\infty}}{\sqrt{m ! n ! \tilde{m} ! \tilde{n} !}}\left\|g_{\sigma}^{n}\right\|_{2}^{2}\left\|g_{\sigma}^{m-1}\right\|_{2}^{2} .
$$

Let us now consider (4.30) for $j=2$. We set $w:=\underline{k^{\prime}}-\underline{\check{r}}$ and define

$$
V\left(\check{k}_{1}, p, q, w\right):=E_{-\breve{k}_{1}+p-w}+E_{\check{k}_{1}+q+w} .
$$

We note that, by disjointness of supports of $h_{1}^{\prime}, h_{2}^{\prime}$, the condition $\bar{h}_{2}^{\prime}\left(-\check{k}_{1}+p-w\right) \bar{h}_{1}^{\prime}\left(\check{k}_{1}+\right.$ $q+w) \neq 0$ implies that

$$
\left|\nabla_{\breve{k}_{1}} V\left(\check{k}_{1}, p, q, w\right)\right| \geq \varepsilon^{\prime}>0,
$$

for some $\varepsilon^{\prime}$ independent of $\breve{k}_{1}, p, q$ and $w$. Thus we can write the following identity

$$
e^{i V\left(\check{k}_{1}, p, q, w\right) t}=\frac{\nabla_{\breve{k}_{1}} V\left(\check{k}_{1}, p, q, w\right) \cdot \nabla_{\check{k}_{1}} e^{i V\left(\check{k}_{1}, p, q, w\right) t}}{i t\left|\nabla_{\breve{k}_{1}} V\left(\check{k}_{1}, p, q, w\right)\right|^{2}} .
$$


Now we define the function

$$
J\left(\check{k}_{1}, p, q, w\right):=\frac{\nabla_{\check{k}_{1}} V\left(\check{k}_{1}, p, q, w\right)}{\left|\nabla_{\check{k}_{1}} V\left(\check{k}_{1}, p, q, w\right)\right|^{2}} \bar{h}_{2}^{\prime}\left(-\check{k}_{1}+p-w\right) \bar{h}_{1}^{\prime}\left(\check{k}_{1}+q+w\right) .
$$

We note that, by Proposition 1.1(a), for any multiindex $\beta$ s.t. $0 \leq|\beta| \leq 1$ there hold the bounds

$$
\left|\partial_{\check{k}_{1}}^{\beta} J\left(\check{k}_{1}, p, q, w\right)\right| \leq c_{0}\left(h_{1}^{\prime}, h_{2}^{\prime}\right), \quad\left|\partial_{\check{k}_{1}}^{\beta} \chi_{j}\left(\check{k}_{1}\right)\right| \leq \frac{c}{\left(\sigma_{\mathrm{s}}^{\prime}\right)^{|\beta|}},
$$

where $c_{0}\left(h_{1}^{\prime}, h_{2}^{\prime}\right)$ has the form

$$
c_{0}\left(h_{1}^{\prime}, h_{2}^{\prime}\right)=c \sum_{\beta_{1}, \beta_{2} ; 0 \leq\left|\beta_{1}\right|+\left|\beta_{2}\right| \leq 1}\left\|\partial^{\beta_{1}} h_{1}^{\prime}\right\|_{\infty}\left\|\partial^{\beta_{2}} h_{2}^{\prime}\right\|_{\infty},
$$

where $\beta_{1}, \beta_{2}$ are multiindices. Moreover, we obtain from Theorem 1.2(c) that

$$
\begin{aligned}
& \left|f_{q, \sigma}^{n}(r)\right| \leq \frac{1}{\sqrt{n !}} g_{\sigma}^{n}(r), \\
& \left|\partial_{\check{k}_{1}}^{\beta} f_{p-\underline{\underline{k}}+\underline{\underline{r}}, \sigma^{\prime}}^{\tilde{z}}(\hat{k}, \check{r})\right| \leq \frac{1}{\sqrt{\tilde{m} !}}\left(\frac{1}{\left(\sigma^{\prime}\right)^{\delta_{\lambda_{0}}}}\right)^{|\beta|} g_{\sigma^{\prime}}^{\tilde{m}}(\hat{k}, \check{r}) \\
& \left|\partial_{\check{k}_{1}}^{\beta}\left(\chi_{2}\left(\check{k}_{1}\right) \bar{f}_{q+\underline{\tilde{n}}-\underline{\underline{r}}, \sigma^{\prime}}(\hat{r}, \check{k}) f_{p, \sigma}^{m}(k)\right)\right| \leq \frac{1}{\sqrt{\tilde{n} ! m !}}\left(\frac{1}{\left(\sigma^{\prime}\right)^{\delta_{\lambda_{0}}}}+\frac{1}{\sigma_{\mathrm{s}}^{\prime}}\right)^{|\beta|} g_{\sigma^{\prime}}^{\tilde{n}}(\hat{r}, \check{k}) g_{\sigma}^{m}(k) \text {. }
\end{aligned}
$$

Making use of the fact that $\chi_{2}\left(\check{k}_{1}\right)$ vanishes for $\left|\check{k}_{1}\right| \leq \sigma^{\prime}$ we integrate by parts in (4.30). We obtain

$$
\begin{aligned}
\check{I}_{m, n, \tilde{m}, \tilde{n}}^{(1)(2)}= & -\frac{1}{i t} \int d^{3} q d^{3} p \int d^{3 n} r d^{3 m} k e^{i\left(V\left(\check{k}_{1}, p, q, w\right)-V(0, p, q, 0)\right) t} h_{2}(p) h_{1}(q) f_{q, \sigma}^{n}(r) \\
& \times \nabla_{\check{k}_{1}} \cdot\left(J\left(\check{k}_{1}, p, q, w\right) \chi_{2}\left(\check{k}_{1}\right) \bar{f}_{q+\underline{\tilde{n}}-\underline{\underline{r}, \sigma^{\prime}}}(\hat{r}, \check{k}) f_{p, \sigma}^{m}(k) \bar{f}_{p-\underline{\tilde{k}}+\underline{\underline{m}}, \sigma^{\prime}}^{\tilde{k}}(\hat{k}, \check{r})\right) .
\end{aligned}
$$

Making use of the bounds (4.37), (4.39), (4.40), (4.41) and, since $\sigma^{\prime} \geq \sigma$, of the fact that $g_{\sigma^{\prime}}^{\tilde{n}}(\hat{r}, \check{k}) \leq g_{\sigma}^{\tilde{n}}(\hat{r}, \check{k})$ we estimate

$$
\left|\check{I}_{m, n, \tilde{m}, \tilde{n}}^{(1)(2)}\right| \leq \frac{1}{t}\left(\frac{1}{\left(\sigma^{\prime}\right)^{\delta_{\lambda_{0}}}}+\frac{1}{\sigma_{\mathrm{s}}^{\prime}}\right) \frac{\left\|h_{1}\right\|_{1}\left\|h_{2}\right\|_{1} c_{0}\left(h_{1}^{\prime}, h_{2}^{\prime}\right)}{\sqrt{m ! n ! \tilde{m} ! \tilde{n} !}}\left\|g_{\sigma}^{n}\right\|_{2}^{2}\left\|g_{\sigma}^{m}\right\|_{2}^{2} .
$$

Exploiting (4.43), (4.32) and the fact that $\sigma_{\mathrm{s}}^{\prime}=\kappa\left(\sigma^{\prime} / \kappa\right)^{1 /\left(8 \gamma_{0}\right)}$, we get (4.28). Here we estimated trivially

$$
\left\|g_{\sigma}^{n}\right\|_{2}^{2}\left\|g_{\sigma}^{m-1}\right\|_{2}^{2}+\left\|g_{\sigma}^{n}\right\|_{2}^{2}\left\|g_{\sigma}^{m}\right\|_{2}^{2} \leq\left(\left\|g_{\sigma}^{n-1}\right\|_{2}^{2}+\left\|g_{\sigma}^{n}\right\|_{2}^{2}\right)\left(\left\|g_{\sigma}^{m-1}\right\|_{2}^{2}+\left\|g_{\sigma}^{m}\right\|_{2}^{2}\right)
$$

to obtain an expression in (4.28) which is symmetric under the substitution $m \leftrightarrow n$. 
Lemma 4.3 Let $\hat{I}_{m, n, \tilde{m}, \tilde{n}}^{(2)}$ be defined as follows

$$
\begin{aligned}
\hat{I}_{m, n, \tilde{m}, \tilde{n}}^{(2)}:= & \int d^{3} q d^{3} p \int d^{3 n} r d^{3 m} k G_{1, n}(q ; r) G_{2, m}(p ; k) \\
& \times \bar{G}_{1, \tilde{n}}^{\prime}(p-\underline{\hat{k}}+\underline{\hat{r}} ; \hat{r}, \check{k}) \bar{G}_{2, \tilde{m}}^{\prime}(q+\underline{\hat{k}}-\underline{\hat{r}} ; \hat{k}, \check{r}),
\end{aligned}
$$

where $G_{i, m}, G_{i, m}^{\prime}, i \in\{1,2\}$, are defined in (3.40), (3.41), the notation $k=(\hat{k}, \breve{k}), r=(\hat{r}, \check{r})$ is explained in Lemma B.2 and we consider a permutation in (3.43) for which $\hat{k}$ or $\hat{r}$ are non-empty. Then there holds

$$
\begin{aligned}
\left|\hat{I}_{m, n, \tilde{m}, \tilde{n}}^{(2)}\right| \leq & \left(\frac{1}{t}\left(\frac{1}{\sigma^{\delta_{\lambda_{0}}}}+\frac{1}{\sigma^{1 /\left(8 \gamma_{0}\right)}}\right)+\left(\sigma^{\prime}\right)^{\bar{\alpha} /\left(4 \gamma_{0}\right)}\right) \frac{C\left(h, h^{\prime}\right)}{\sqrt{m ! n ! \tilde{m} ! \tilde{n} !}} \\
& \times\left(\left\|g_{\sigma}^{n-1}\right\|_{2}^{2}+\left\|g_{\sigma}^{n}\right\|_{2}^{2}\right)\left(\left\|g_{\sigma}^{m-1}\right\|_{2}^{2}+\left\|g_{\sigma}^{m}\right\|_{2}^{2}\right),
\end{aligned}
$$

where $C\left(h, h^{\prime}\right):=c\left\|h_{1}\right\|_{1}\left\|h_{2}\right\|_{1} \sum_{\beta_{1}, \beta_{2} ; 0 \leq\left|\beta_{1}\right|+\left|\beta_{2}\right| \leq 1}\left\|\partial^{\beta_{1}} h_{1}^{\prime}\right\|_{\infty}\left\|\partial^{\beta_{2}} h_{2}^{\prime}\right\|_{\infty}$ and the sum extends over multiindices $\beta_{1}, \beta_{2}$. We note that for $\hat{k}$ (resp. $\left.\hat{r}\right)$ non-empty we have $m \neq 0($ resp. $n \neq 0)$ and there always holds $m+n=\tilde{m}+\tilde{n}$. We set by convention $g_{\sigma}^{-1}=0$.

Proof By inserting the definitions of $G_{i, m}, G_{i, m}^{\prime}$ we obtain

$$
\begin{aligned}
\hat{I}_{m, n, \tilde{m}, \tilde{n}}^{(2)}= & \int d^{3} q d^{3} p \int d^{3 n} r d^{3 m} k e^{i\left(E_{q+\underline{\hat{k}}-\underline{\hat{r}}}-E_{q}\right) t} e^{i\left(E_{p-\underline{\hat{k}}+\underline{\hat{r}}}-E_{p}\right) t} h_{2}(p) h_{1}(q) \\
& \times \bar{h}_{2}^{\prime}(q+\underline{\hat{k}}-\underline{\hat{r}}) \bar{h}_{1}^{\prime}(p-\underline{\hat{k}}+\underline{\hat{r}}) f_{q, \sigma}^{n}(r) \bar{f}_{q+\underline{\tilde{k}}-\underline{\hat{r}}, \sigma^{\prime}}(\hat{k}, \check{r}) f_{p, \sigma}^{m}(k) \bar{f}_{p-\underline{\hat{n}}+\underline{\hat{r}}, \sigma^{\prime}}^{\tilde{n}}(\hat{r}, \check{k}) .
\end{aligned}
$$

We note that by substitutions $h_{1}^{\prime} \leftrightarrow h_{2}^{\prime}, \tilde{m} \leftrightarrow \tilde{n}, \hat{k} \leftrightarrow \check{k}, \hat{r} \leftrightarrow \check{r}$ we obtain formula (4.29). Now the statement follows from Lemma 4.2 and the fact that (4.28) does not change under the above substitutions.

Acknowledgements The authors are grateful to J. Fröhlich for the unpublished notes that have inspired this paper. We also thank the Hausdorff Research Institute for Mathematics, Bonn, for hospitality at final stages of this work. W.D. acknowledges hospitality of the University of California Davis, where this collaboration started.

A.P. is supported by the NSF grant \#DMS-0905988. W.D. is supported by the German Research Foundation (DFG) within the grant SP181/25-2 and stipend DY107/1-1. Moreover, W.D. would like to acknowledge the support of the Danish Council for Independent Research, grant no. 09-065927 "Mathematical Physics", and of the Lundbeck Foundation.

\section{Appendix A: Domain Questions}

Lemma A.1 Let $n \in \mathbb{N}$. There exist constants $0 \leq a<1$ and $b \geq 0$ s.t. for any $\Psi \in \mathcal{C}^{(n)}$ there holds the bound

$$
\left\|H_{\mathrm{I}}^{(n)} \Psi\right\| \leq a\left\|H_{\mathrm{fr}}^{(n)} \Psi\right\|+b\|\Psi\|,
$$

where $H_{\mathrm{I}}^{(n)}=\left.H_{\mathrm{I}}\right|_{\mathcal{C}^{(n)}}, H_{\mathrm{fr}}^{(n)}=\left.H_{\mathrm{fr}}\right|_{\mathcal{C}^{(n)}}$. 
Proof Let us use the form of the interaction Hamiltonian appearing in formula (1.33). We have $H_{\mathrm{I}}^{(n)}=H_{\mathrm{I}}^{\mathrm{a},(n)}+H_{\mathrm{I}}^{\mathrm{c},(n)}$, where

$$
H_{\mathrm{I}}^{\mathrm{a},(n)}:=\sum_{i=1}^{n} \int d^{3} k v_{\bar{\alpha}}(k) e^{i k x_{i}} a(k)
$$

and $H_{\mathrm{I}}^{\mathrm{c},(n)}=\left(H_{\mathrm{I}}^{\mathrm{a},(n)}\right)^{*}$. Let us set $C_{n}(k):=\sum_{l=1}^{n} e^{i k x_{l}}$ and compute for some $\Psi \in \mathcal{C}^{(n)}$

$$
\begin{aligned}
\left\|H_{\mathrm{I}}^{\mathrm{a},(n)} \Psi\right\| & \leq \int d^{3} k v_{\bar{\alpha}}(k)\left\|C_{n}(k) a(k) \Psi\right\| \leq n \int d^{3} k v_{\bar{\alpha}}(k)\|a(k) \Psi\| \\
& \leq n\left\|\omega^{-1 / 2} v_{\bar{\alpha}}\right\|_{2}\left\langle\Psi, H_{\mathrm{f}} \Psi\right\rangle^{\frac{1}{2}} \leq \frac{1}{4}\left\|H_{\mathrm{fr}} \Psi\right\|+n^{2}\left\|\omega^{-1 / 2} v_{\bar{\alpha}}\right\|_{2}^{2}\|\Psi\|,
\end{aligned}
$$

where in the last step we anticipate that (A.1) should hold with $0<a<1$.

Let us now consider the creation part of $H_{\mathrm{I}}^{(n)}$. Making use of the canonical commutation relations, we get

$$
\begin{aligned}
\left\|H_{\mathrm{I}}^{\mathrm{c},(n)} \Psi\right\|^{2} & =\int d^{3} k_{1} d^{3} k_{2} v_{\bar{\alpha}}\left(k_{1}\right) v_{\bar{\alpha}}\left(k_{2}\right)\left\langle C_{n}\left(k_{1}\right)^{*} a^{*}\left(k_{1}\right) \Psi, C_{n}\left(k_{2}\right)^{*} a^{*}\left(k_{2}\right) \Psi\right\rangle \\
& \leq\left\|H_{\mathrm{I}}^{\mathrm{a},(n)} \Psi\right\|^{2}+n^{2}\left\|v_{\bar{\alpha}}\right\|_{2}^{2}\|\Psi\|^{2},
\end{aligned}
$$

which, together with (A.3), concludes the proof.

Lemma A.2 The domain $\mathcal{D}$, defined in (2.19), is contained in the domains of $H, H_{\mathrm{e}}, H_{\mathrm{f}}$, $H_{\mathrm{I}}^{\mathrm{a} / \mathrm{c}}, \check{H}_{\mathrm{I}, \sigma}$ and $\hat{\eta}^{*}(h), h \in C_{0}^{2}(S)$. Moreover, these operators leave $\mathcal{D}$ invariant.

Proof Let $\Psi_{l}^{r_{1}, r_{2}}$ be a vector of the form (2.17). Then

$$
\begin{aligned}
\hat{\eta}_{\sigma}^{*}(h) \Psi_{l}^{r_{1}, r_{2}} & \\
= & \sum_{m, m^{\prime}} \frac{1}{\sqrt{m^{\prime} !}} \frac{1}{\sqrt{m !}} \int d^{3} p^{\prime} d^{3 l} p d^{3 m^{\prime}} k^{\prime} d^{3 m} k F_{1, m^{\prime}}^{\prime}\left(p^{\prime} ; k^{\prime}\right) F_{l, m}(p ; k) \\
& \times \eta^{*}\left(p^{\prime}\right) \eta^{*}(p)^{l} a^{*}\left(k^{\prime}\right)^{m^{\prime}} a^{*}(k)^{m} \Omega \\
= & \sum_{\tilde{m}=0}^{\infty} \frac{1}{\sqrt{\tilde{m} !}} \int d^{3(l+1)} \tilde{p} d^{3 \tilde{m}} \tilde{k} \tilde{F}_{\tilde{m}}(\tilde{p} ; \tilde{k}) \eta^{*}(\tilde{p})^{l+1} a^{*}(\tilde{k})^{\tilde{m}} \Omega,
\end{aligned}
$$

where $F_{1, m^{\prime}}^{\prime}\left(p^{\prime}, k^{\prime}\right):=h\left(p^{\prime}+\underline{k}^{\prime}\right) f_{p^{\prime}+\underline{k}, \sigma}^{m^{\prime}}\left(k^{\prime}\right), \tilde{m}:=m+m^{\prime}, \tilde{k}:=\left(k, k^{\prime}\right), \tilde{p}:=\left(p, p^{\prime}\right)$ and

$$
\tilde{F}_{\tilde{m}}(\tilde{p} ; \tilde{k})=\sum_{m=0}^{\tilde{m}} \frac{\sqrt{\tilde{m} !}}{\sqrt{(\tilde{m}-m) !} \sqrt{m !}}\left(F_{1,(\tilde{m}-m)}^{\prime} F_{l, m}\right)_{\mathrm{sym}}(\tilde{p} ; \tilde{k}),
$$

where the symmetrization is performed in the $\tilde{p}$ and $\tilde{k}$ variables separately. By Theorem 1.2, $F_{1, m^{\prime}}^{\prime}$ satisfies the bound (2.18), and therefore

$$
\left\|\tilde{F}_{\tilde{m}}\right\|_{2} \leq \frac{c^{\tilde{m}}}{\sqrt{\tilde{m} !}},
$$

for some constant $c$. Hence $\hat{\eta}_{\sigma}^{*}(h) \Psi_{l}^{r_{1}, r_{2}}$ is well defined and belongs to $\mathcal{D}$. 
Next, we note that

$$
\begin{aligned}
& H_{\mathrm{e}} \Psi_{l}^{r_{1}, r_{2}}=\sum_{m=0}^{\infty} \frac{1}{\sqrt{m !}} \int d^{3 l} p d^{3 m} k\left(\Omega\left(p_{1}\right)+\cdots+\Omega\left(p_{l}\right)\right) F_{l, m}(p ; k) \eta^{*}(p)^{l} a^{*}(k)^{m} \Omega, \\
& H_{\mathrm{f}} \Psi_{l}^{r_{1}, r_{2}}=\sum_{m=0}^{\infty} \frac{1}{\sqrt{m !}} \int d^{3 l} p d^{3 m} k\left(\omega\left(k_{1}\right)+\cdots+\omega\left(k_{m}\right)\right) F_{l, m}(p ; k) \eta^{*}(p)^{l} a^{*}(k)^{m} \Omega .
\end{aligned}
$$

Due to the support properties of $F_{l, m}(p ; k)$ these vectors are well defined and belong to $\mathcal{D}$.

Finally, we consider the operators $H_{\mathrm{I}}^{\mathrm{a} / \mathrm{c}}$. We recall that the interaction Hamiltonian restricted to $\mathcal{H}^{(l)}$ has the form

$$
H_{\mathrm{I}}^{(l)}:=\sum_{i=1}^{l} \int d^{3} k v_{\bar{\alpha}}(k)\left(e^{i k x_{i}} a(k)+e^{-i k x_{i}} a^{*}(k)\right)
$$

and we express $\Psi_{l}^{r_{1}, r_{2}}$ in terms of its $m$-photon components, i.e.,

$$
\left\{\Psi_{l}^{r_{1}, r_{2}}\right\}^{(l, m)}\left(p_{1}, \ldots, p_{l} ; k_{1}, \ldots, k_{m}\right)=F_{l, m}\left(p_{1}, \ldots, p_{l} ; k_{1}, \ldots, k_{m}\right) .
$$

Now we can write

$$
\begin{aligned}
\left(H_{\mathrm{I}}^{\mathrm{a}} \Psi_{l}^{r_{1}, r_{2}}\right)^{(l, m)}\left(p_{1}, \ldots, p_{l} ; k_{1}, \ldots, k_{m}\right) \\
=\sqrt{m+1} \int d^{3} k v_{\bar{\alpha}}(k) \sum_{i=1}^{l}\left(\Psi_{l}^{r_{1}, r_{2}}\right)^{(l, m+1)} \\
\quad \times\left(p_{1}, \ldots, p_{i}-k, \ldots, p_{l} ; k, k_{1}, \ldots, k_{m}\right) .
\end{aligned}
$$

It is easy to see that for some constant $c$, independent of $m$,

$$
\left\|\left(H_{\mathrm{I}}^{\mathrm{a}} \Psi_{l}^{r_{1}, r_{2}}\right)^{(l, m)}\right\|_{2} \leq \frac{c^{m}}{\sqrt{m !}} .
$$

Similarly, we obtain that

$$
\begin{aligned}
& \left(H_{\mathrm{I}}^{\mathrm{c}} \Psi_{l}^{r_{1}, r_{2}}\right)^{(l, m)}\left(p_{1}, \ldots, p_{l} ; k_{1}, \ldots, k_{m}\right) \\
& =\frac{1}{\sqrt{m}} \sum_{i=1}^{m} \sum_{j=1}^{l} v_{\bar{\alpha}}\left(k_{i}\right)\left(\Psi_{l}^{r_{1}, r_{2}}\right)^{(l, m-1)} \\
& \quad \times\left(p_{1}, \ldots, p_{j}+k_{i}, \ldots, p_{l} ; k_{1}, \ldots, k_{i_{*}}, \ldots, k_{m}\right),
\end{aligned}
$$

where $k_{i_{*}}$ means omission of the $i$-th variable. This gives, again, a bound of the form (A.13). Since the case of $\check{H}_{\mathrm{I}, \sigma}$ is analogous, this concludes the proof. 


\section{Appendix B: Fock Space Combinatorics}

In Lemmas B.1, B.3 and B.5 below we deal first with $G_{i, m}, G_{i, m}^{\prime}, F_{n, m}, F_{n, m}^{\prime}$ of Schwartz class and then extend the results to square integrable functions using Theorem X.44 of [26].

Lemma B.1 Let $G_{m}, G_{m}^{\prime} \in L^{2}\left(\mathbb{R}^{3} \times \mathbb{R}^{3 m}\right)$ be symmetric in their photon variables, see (1.68). Let us define as operators on $\mathcal{C}$

$$
B_{m}^{*}\left(G_{m}\right):=\int d^{3} p d^{3 m} k G_{m}(p ; k) \eta^{*}(p-\underline{k}) a^{*}(k)^{m}
$$

and $B_{m}\left(G_{m}\right):=\left(B_{m}^{*}\left(G_{m}\right)\right)^{*}$. Then there holds the identity

$$
\left\langle\Omega, B_{m}\left(G_{m}^{\prime}\right) B_{m}^{*}\left(G_{m}\right) \Omega\right\rangle=m ! \int d^{3} p d^{3 m} k \bar{G}_{m}^{\prime}(p ; k) G_{m}(p ; k)
$$

Proof We compute

$$
\begin{aligned}
\langle\Omega, & \left.B_{m}\left(G_{m}^{\prime}\right) B_{m}^{*}\left(G_{m}\right) \Omega\right\rangle \\
= & \int d^{3} p d^{3 m} k \int d^{3} p^{\prime} d^{3 m} k^{\prime} G_{m}(p ; k) \bar{G}_{m}^{\prime}\left(p^{\prime} ; k^{\prime}\right) \\
& \times\left\langle\Omega, a\left(k^{\prime}\right)^{m} \eta\left(p^{\prime}-\underline{k}^{\prime}\right) \eta^{*}(p-\underline{k}) a^{*}(k)^{m} \Omega\right\rangle \\
= & \int d^{3} p d^{3 m} k \int d^{3} p^{\prime} d^{3 m} k^{\prime} G_{m}(p ; k) \bar{G}_{m}^{\prime}\left(p^{\prime} ; k^{\prime}\right) \delta\left(p-\underline{k}-p^{\prime}+\underline{k}^{\prime}\right) \\
& \times\left\langle\Omega, a\left(k^{\prime}\right)^{m} a^{*}(k)^{m} \Omega\right\rangle \\
= & \int d^{3} p d^{3 m} k \int d^{3 m} k^{\prime} G_{m}(p ; k) \bar{G}_{m}^{\prime}\left(p-\underline{k}+\underline{k}^{\prime} ; k^{\prime}\right)\left\langle\Omega, a\left(k^{\prime}\right)^{m} a^{*}(k)^{m} \Omega\right\rangle \\
= & \int d^{3} p d^{3 m} k \int d^{3 m} k^{\prime} G_{m}(p ; k) \bar{G}_{m}^{\prime}\left(p-\underline{k}+\underline{k}^{\prime} ; k^{\prime}\right) \sum_{\rho \in S_{m}} \prod_{i=1}^{m} \delta\left(k_{\rho(i)}-k_{i}^{\prime}\right) \\
= & m ! \int d^{3} p d^{3 m} k G_{m}(p ; k) \bar{G}_{m}^{\prime}(p ; k),
\end{aligned}
$$

where $S_{m}$ is the set of all permutations of an $m$-element set and in the last step we exploited the fact that $G_{m}^{\prime}$ is symmetric in its photon variables.

Lemma B.2 Let $n, m, \tilde{n}, \tilde{m} \in \mathbb{N}_{0}$ be s.t. $n+m=\tilde{n}+\tilde{m}$. Let us choose

$$
\begin{array}{ll}
r=\left(r_{1}, \ldots, r_{n}\right) \in \mathbb{R}^{3 n}, & k=\left(k_{1}, \ldots, k_{m}\right) \in \mathbb{R}^{3 m}, \\
\tilde{r}=\left(\tilde{r}_{1}, \ldots, \tilde{r}_{\tilde{n}}\right) \in \mathbb{R}^{3 \tilde{n}}, & \tilde{k}=\left(\tilde{k}_{1}, \ldots, \tilde{k}_{\tilde{m}}\right) \in \mathbb{R}^{3 \tilde{m}}
\end{array}
$$

and define the sets

$$
\begin{array}{ll}
C_{n}:=\{1, \ldots, n\}, & C_{n}^{\prime}:=\{n+1, \ldots, n+m\}, \\
C_{\tilde{n}}:=\{1, \ldots, \tilde{n}\}, & C_{\tilde{n}}^{\prime}:=\{\tilde{n}+1, \ldots, \tilde{n}+\tilde{m}\} .
\end{array}
$$


(Note that $C_{n}^{\prime}$ is the complement of $C_{n}$ in $\{1, \ldots, n+m\}$. Similarly for $C_{\tilde{n}}^{\prime}$ ). Let $S_{m+n}$ be the set of permutations of an $m+n$ element set. For any $\rho \in S_{m+n}$ we introduce the following notation:

$$
\begin{array}{ll}
\hat{r}:=\left(r_{i}\right)_{(i, \rho(i)) \in C_{n} \times C_{\tilde{n}}}, & \check{r}:=\left(r_{i}\right)_{(i, \rho(i)) \in C_{n} \times C_{\tilde{n}}^{\prime},} \\
\hat{k}:=\left(k_{i-n}\right)_{(i, \rho(i)) \in C_{n}^{\prime} \times C_{\tilde{n}}^{\prime},} & \breve{k}:=\left(k_{i-n}\right)_{(i, \rho(i)) \in C_{n}^{\prime} \times C_{\tilde{n}}},
\end{array}
$$

so that $r=(\hat{r}, \check{r}), k=(\hat{k}, \check{k})$. Similarly,

$$
\begin{array}{ll}
\hat{\tilde{r}}:=\left(\tilde{r}_{\rho(i)}\right)_{(i, \rho(i)) \in C_{n} \times C_{\tilde{n}}}, & \check{\tilde{r}}:=\left(\tilde{r}_{\rho(i)}\right)_{(i, \rho(i)) \in C_{n}^{\prime} \times C_{\tilde{n}},} \\
\hat{\tilde{k}}:=\left(\tilde{k}_{\rho(i)-\tilde{n}}\right)_{(i, \rho(i)) \in C_{n}^{\prime} \times C_{\tilde{n}}^{\prime}}, & \check{\tilde{k}}:=\left(\tilde{k}_{\rho(i)-\tilde{n}}\right)_{(i, \rho(i)) \in C_{n} \times C_{\tilde{n}}^{\prime}},
\end{array}
$$

so that $\tilde{r}=(\hat{\tilde{r}}, \check{\tilde{r}})$ and $\tilde{k}=(\hat{\tilde{k}}, \tilde{\tilde{k}}) .\left(\right.$ If $\left\{i \mid(i, \rho(i)) \in C_{n} \times C_{\tilde{n}}\right\}=\emptyset$ then we say that $\hat{r}$ is empty, and analogously for other collections of photon variables introduced above). Finally, we define

$$
\begin{aligned}
& \delta(\hat{r}-\hat{\tilde{r}}):=\prod_{(i, \rho(i)) \in C_{n} \times C_{\tilde{n}}} \delta\left(r_{i}-\tilde{r}_{\rho(i)}\right), \\
& \delta(\check{r}-\check{\tilde{k}}):=\prod_{(i, \rho(i)) \in C_{n} \times C_{\tilde{n}}^{\prime}} \delta\left(r_{i}-\tilde{k}_{\rho(i)-\tilde{n}}\right), \\
& \delta(\check{k}-\check{\tilde{r}}):=\prod_{(i, \rho(i)) \in C_{n}^{\prime} \times C_{\tilde{n}}} \delta\left(k_{i-n}-\tilde{r}_{\rho(i)}\right), \\
& \delta(\hat{k}-\hat{\tilde{k}}):=\prod_{(i, \rho(i)) \in C_{n}^{\prime} \times C_{\tilde{n}}^{\prime}} \delta\left(k_{i-n}-\tilde{k}_{\rho(i)-\tilde{n}}\right) .
\end{aligned}
$$

Then there holds

$$
\left\langle\Omega, a(\tilde{r})^{\tilde{n}} a(\tilde{k})^{\tilde{m}} a^{*}(r)^{n} a^{*}(k)^{m} \Omega\right\rangle=\sum_{\rho \in S_{m+n}} \delta(\hat{r}-\hat{\tilde{r}}) \delta(\check{r}-\tilde{\tilde{k}}) \delta(\check{k}-\check{\tilde{r}}) \delta(\hat{k}-\hat{\tilde{k}}) .
$$

Proof Let $\left(v_{1}, \ldots, v_{n+m}\right)=\left(r_{1}, \ldots, r_{n}, k_{1}, \ldots, k_{m}\right)$ and $\left(\tilde{v}_{1}, \ldots, \tilde{v}_{n+m}\right)=\left(\tilde{r}_{1}, \ldots, \tilde{r}_{\tilde{n}}\right.$, $\left.\tilde{k}_{1}, \ldots, \tilde{k}_{\tilde{m}}\right)$. There holds

$$
\begin{aligned}
\langle\Omega, & \left.a(\tilde{r})^{\tilde{n}} a(\tilde{k})^{\tilde{m}} a^{*}(r)^{n} a^{*}(k)^{m} \Omega\right\rangle \\
= & \sum_{\rho \in S_{m+n}} \prod_{j=1}^{m+n} \delta\left(v_{j}-\tilde{v}_{\rho(j)}\right) \\
= & \sum_{\rho \in S_{m+n}} \delta\left(r_{1}-\tilde{v}_{\rho(1)}\right) \cdots \delta\left(r_{n}-\tilde{v}_{\rho(n)}\right) \delta\left(k_{1}-\tilde{v}_{\rho(n+1)}\right) \cdots \delta\left(k_{m}-\tilde{v}_{\rho(n+m)}\right) \\
= & \sum_{\rho \in S_{m+n}}\left(\prod_{(i, \rho(i)) \in C_{n} \times C_{\tilde{n}}} \delta\left(r_{i}-\tilde{r}_{\rho(i)}\right)\right)\left(\prod_{(i, \rho(i)) \in C_{n} \times C_{\tilde{n}}^{\prime}} \delta\left(r_{i}-\tilde{k}_{\rho(i)-\tilde{n}}\right)\right) \\
& \times\left(\prod_{(i, \rho(i)) \in C_{n}^{\prime} \times C_{\tilde{n}}} \delta\left(k_{i-n}-\tilde{r}_{\rho(i)}\right)\right)\left(\prod_{(i, \rho(i)) \in C_{n}^{\prime} \times C_{\tilde{n}}^{\prime}} \delta\left(k_{i-n}-\tilde{k}_{\rho(i)-\tilde{n}}\right)\right),
\end{aligned}
$$

which concludes the proof. 
Lemma B.3 Let $F_{n, m}, F_{n, m}^{\prime} \in L^{2}\left(\left(\mathbb{R}^{3} \times \mathbb{R}^{3 n}\right) \times\left(\mathbb{R}^{3} \times \mathbb{R}^{3 m}\right)\right)$ be symmetric in the photon variables for any $n, m \in \mathbb{N}$. Let us introduce the following operators on $\mathcal{C}$

$$
B_{n, m}^{*}\left(F_{n, m}\right):=\int d^{3} q d^{3} p \int d^{3 n} r d^{3 m} k F_{n, m}(q ; r \mid p ; k) a^{*}(r)^{n} a^{*}(k)^{m} \eta^{*}(p-\underline{k}) \eta^{*}(q-\underline{r})
$$

and set $B_{n, m}\left(F_{n, m}\right):=\left(B_{n, m}^{*}\left(F_{n, m}\right)\right)^{*}$. There holds

$$
\begin{aligned}
& \left\langle B_{\tilde{n}, \tilde{m}}^{*}\left(F_{\tilde{n}, \tilde{m}}^{\prime}\right) \Omega, B_{n, m}^{*}\left(F_{n, m}\right) \Omega\right\rangle \\
& =\sum_{\rho \in S_{m+n}} \int d^{3} q d^{3} p \int d^{3 n} r d^{3 m} k F_{n, m}(q ; r \mid p ; k) \\
& \quad \times\left(\bar{F}_{\tilde{n}, \tilde{m}}^{\prime}(p-\underline{\hat{k}}+\underline{\hat{r}} ; \hat{r}, \check{k} \mid q+\underline{\hat{k}}-\underline{\hat{r}} ; \hat{k}, \check{r})\right. \\
& \left.\quad+\bar{F}_{\tilde{n}, \tilde{m}}^{\prime}(q+\underline{\underline{k}}-\underline{\check{r}} ; \hat{r}, \check{k} \mid p-\underline{\underline{k}}+\underline{\underline{r}} ; \hat{k}, \check{r})\right)
\end{aligned}
$$

for any $\tilde{n}, \tilde{m} \in \mathbb{N}$ s.t. $n+m=\tilde{n}+\tilde{m}$. Otherwise the expression on the l.h.s. is zero. Here $S_{m+n}$ is the set of permutations of an $m+n$ element set and the notation $\hat{k}, \breve{k}, \hat{r}, \check{r}$ is explained in Lemma B.2.

Proof We compute the expectation value

$$
\begin{aligned}
& \left\langle B_{\tilde{n}, \tilde{m}}^{*}\left(F_{\tilde{n}, \tilde{m}}^{\prime}\right) \Omega, B_{n, m}^{*}\left(F_{n, m}\right) \Omega\right\rangle \\
& =\int d^{3} \tilde{q} d^{3} \tilde{p} d^{3} q d^{3} p \int d^{3 \tilde{n}} \tilde{r} d^{3 \tilde{m}} \tilde{k} d^{3 n} r d^{3 m} k \bar{F}_{\tilde{n}, \tilde{m}}^{\prime}(\tilde{q} ; \tilde{r} \mid \tilde{p} ; \tilde{k}) F_{n, m}(q ; r \mid p ; k) \\
& \quad \times(\delta(\tilde{q}-p+\underline{\tilde{k}}-\underline{r})+\delta(\tilde{q}-q-\underline{\tilde{r}}+\underline{r})) \delta(\tilde{p}+\tilde{q}-p-q) \\
& \quad \times\left\langle\Omega, a(\tilde{r})^{\tilde{n}} a(\tilde{k})^{\tilde{m}} a^{*}(r)^{n} a^{*}(k)^{m} \Omega\right\rangle .
\end{aligned}
$$

The last factor is non-zero only if $\tilde{n}+\tilde{m}=n+m$. Moreover,

$$
\left\langle\Omega, a(\tilde{r})^{\tilde{n}} a(\tilde{k})^{\tilde{m}} a^{*}(r)^{n} a^{*}(k)^{m} \Omega\right\rangle=\sum_{\rho \in S_{m+n}} \delta(\hat{r}-\hat{\tilde{r}}) \delta(\check{r}-\check{\tilde{k}}) \delta(\check{k}-\check{\tilde{r}}) \delta(\hat{k}-\hat{\tilde{k}}),
$$

where we made use of Lemma B.2. Thus the r.h.s. of (B.22) is a sum over $\rho \in S_{m+n}$ of terms of the form:

$$
\begin{aligned}
\int d^{3} \tilde{q} d^{3} \tilde{p} d^{3} q d^{3} p \int d^{3 n} r d^{3 m} k \bar{F}_{\tilde{n}, \tilde{m}}^{\prime}(\tilde{q} ; \hat{r}, \check{k} \mid \tilde{p} ; \hat{k}, \check{r}) F_{n, m}(q ; r \mid p ; k) \\
\quad \times \delta(\tilde{p}+\tilde{q}-p-q)(\delta(\tilde{q}-p+\underline{\hat{k}}-\underline{\hat{r}})+\delta(\tilde{q}-q-\underline{\underline{k}}+\underline{\underline{r}})) \\
=\int d^{3} q d^{3} p \int d^{3 n} r d^{3 m} k F_{n, m}(q ; r \mid p ; k)\left(\bar{F}_{\tilde{n}, \tilde{m}}^{\prime}(p-\underline{\hat{k}}+\underline{\hat{r}} ; \hat{r}, \check{k} \mid q+\underline{\hat{k}}-\underline{\hat{r}} ; \hat{k}, \check{r})\right. \\
\left.\quad+\bar{F}_{\tilde{n}, \tilde{m}}^{\prime}(q+\check{\underline{k}}-\underline{\check{r}} ; \hat{r}, \check{k} \mid p-\underline{\check{k}}+\underline{\check{r}} ; \hat{k}, \check{r})\right)
\end{aligned}
$$

which concludes the proof. 
Lemma B.4 Let $G_{1, m}, G_{1, m}^{\prime}, G_{2, m}, G_{2, m}^{\prime} \in L^{2}\left(\mathbb{R}^{3} \times \mathbb{R}^{3 m}\right)$ be symmetric in the photon variables for any $m \in \mathbb{N}$. We define, as an operator on $\mathcal{C}$,

$$
B_{m}^{*}\left(G_{i, m}\right):=\int d^{3} p d^{3 m} k G_{i, m}(p ; k) \eta^{*}(p-\underline{k}) a^{*}(k)^{m}
$$

and set $B_{m}\left(G_{i, m}\right)=\left(B_{m}^{*}\left(G_{i, m}\right)\right)^{*}$. There holds the identity

$$
\begin{aligned}
\langle\Omega, & \left.B_{\tilde{n}}\left(G_{1, \tilde{n}}^{\prime}\right) B_{\tilde{m}}\left(G_{2, \tilde{m}}^{\prime}\right) B_{n}^{*}\left(G_{1, n}\right) B_{m}^{*}\left(G_{2, m}\right) \Omega\right\rangle \\
= & \sum_{\rho \in S_{m+n}} \int d^{3} q d^{3} p \int d^{3 n} r d^{3 m} k G_{1, n}(q ; r) G_{2, m}(p ; k) \\
& \times\left(\bar{G}_{1, \tilde{n}}^{\prime}(p-\underline{\hat{k}}+\underline{\hat{r}} ; \hat{r}, \check{k}) \bar{G}_{2, \tilde{m}}^{\prime}(q+\underline{\hat{k}}-\underline{\hat{r}} ; \hat{k}, \check{r})\right. \\
& \left.+\bar{G}_{1, \tilde{n}}^{\prime}(q+\underline{\check{k}}-\underline{\check{r}} ; \hat{r}, \check{k}) \bar{G}_{2, \tilde{m}}^{\prime}(p-\underline{\breve{k}}+\underline{\check{r}} ; \hat{k}, \check{r})\right),
\end{aligned}
$$

for any $n, \tilde{n}, \tilde{m} \in \mathbb{N}$ s.t. $n+m=\tilde{n}+\tilde{m}$. Otherwise the expression on the l.h.s. is zero. Here $S_{m+n}$ is the set of permutations of an $m+n$ element set and the notation $\hat{k}, \check{k}, \hat{r}, \check{r}$ is explained in Lemma B.2.

Proof Follows immediately from Lemma B.3.

Lemma B.5 Let $G_{1, m}, G_{2, m} \in L^{2}\left(\mathbb{R}^{3} \times \mathbb{R}^{3 m}\right)$ be supported in $\mathbb{R}^{3} \times\left\{k \in \mathbb{R}^{3}|| k \mid \geq \sigma\right\}^{\times m}$ and symmetric in their photon variables. There holds the identity

$$
\begin{aligned}
& \left\langle\Omega, B_{\tilde{n}}\left(G_{2, \tilde{n}}\right)\left(\check{H}_{\mathrm{I}, \sigma}^{\mathrm{c}}\right)^{*} B_{\tilde{m}}\left(G_{1, \tilde{m}}\right) B_{n}^{*}\left(G_{1, n}\right) \check{H}_{\mathrm{I}, \sigma}^{\mathrm{c}} B_{m}^{*}\left(G_{2, m}\right) \Omega\right\rangle \\
& =\sum_{\rho \in S_{m+n}} \int d^{3} q d^{3} p \int d^{3 n} r d^{3 m} k G_{1, n}(q ; r) G_{2, m}(p ; k) \\
& \quad \times\left(\int d^{3} \tilde{p} \check{v}_{\tilde{\alpha}}^{\sigma}(\tilde{p})^{2} \bar{G}_{2, \tilde{n}}(p-\tilde{p}-\underline{\hat{k}}+\underline{\hat{r}} ; \hat{r}, \check{k}) \bar{G}_{1, \tilde{m}}(\tilde{p}+q+\underline{\hat{k}}-\underline{\hat{r}} ; \hat{k}, \check{r})\right. \\
& \left.\quad+\left\|\check{v}_{\bar{\alpha}}^{\sigma}\right\|_{2}^{2} \bar{G}_{2, \tilde{n}}(q+\underline{\underline{k}}-\underline{\underline{r}} ; \hat{r}, \check{k}) \bar{G}_{1, \tilde{m}}(p-\underline{\underline{k}}+\underline{\check{r}} ; \hat{k}, \check{r})\right)
\end{aligned}
$$

for $m+n=\tilde{m}+\tilde{n}$, otherwise the l.h.s. is zero. Here $S_{m+n}$ is the set of permutations of an $m+n$ element set and the notation $\hat{k}, \breve{k}, \hat{r}, \check{r}$ is explained in Lemma B.2.

Proof We compute the expectation value

$$
\begin{aligned}
\langle\Omega, & \left.B_{\tilde{n}}\left(G_{2, \tilde{n}}\right)\left(\check{H}_{\mathrm{I}, \sigma}^{\mathrm{c}}\right)^{*} B_{\tilde{m}}\left(G_{1, \tilde{m}}\right) B_{n}^{*}\left(G_{1, n}\right) \check{H}_{\mathrm{I}, \sigma}^{\mathrm{c}} B_{m}^{*}\left(G_{2, m}\right) \Omega\right\rangle \\
= & \int d^{3} \tilde{u} d^{3} \tilde{w} d^{3} u d^{3} w \int d^{3} \tilde{q} d^{3} \tilde{p} d^{3} q d^{3} p \int d^{3 \tilde{n}} \tilde{r} d^{3 \tilde{m}} \tilde{k} d^{3 n} r d^{3 m} k \\
& \times \check{v}_{\bar{\alpha}}^{\sigma}(\tilde{w}) \check{v}_{\bar{\alpha}}^{\sigma}(w) \bar{G}_{2, \tilde{n}}(\tilde{q} ; \tilde{r}) \bar{G}_{1, \tilde{m}}(\tilde{p} ; \tilde{k}) G_{1, n}(q ; r) G_{2, m}(p ; k) \\
& \times\left\langle\Omega, \eta(\tilde{p}-\tilde{k}) \eta^{*}(\tilde{u}) \eta(\tilde{u}-\tilde{w}) \eta(\tilde{q}-\underline{\tilde{r}}) \eta^{*}(q-\underline{r}) \eta^{*}(u-w) \eta(u) \eta^{*}(p-\underline{k}) \Omega\right\rangle \\
& \times\left\langle\Omega, a(\tilde{r})^{\tilde{n}} a(\tilde{w}) a(\tilde{k})^{\tilde{m}} a^{*}(r)^{n} a^{*}(w) a^{*}(k)^{m} \Omega\right\rangle .
\end{aligned}
$$


We note that

$$
\begin{aligned}
\langle\Omega, & \left.\eta(\tilde{p}-\underline{\tilde{k}}) \eta^{*}(\tilde{u}) \eta(\tilde{u}-\tilde{w}) \eta(\tilde{q}-\underline{\tilde{r}}) \eta^{*}(q-\underline{r}) \eta^{*}(u-w) \eta(u) \eta^{*}(p-\underline{k}) \Omega\right\rangle \\
= & \delta(\tilde{p}-\underline{\tilde{k}}-\tilde{u}) \delta(p-\underline{k}-u)\left\langle\Omega, \eta(\tilde{u}-\tilde{w}) \eta(\tilde{q}-\underline{\tilde{r}}) \eta^{*}(q-\underline{r}) \eta^{*}(u-w) \Omega\right\rangle \\
= & \delta(\tilde{p}-\underline{\tilde{k}}-\tilde{u}) \delta(p-\underline{k}-u)(\delta(\tilde{u}-\tilde{w}-q+\underline{r}) \delta(\tilde{q}-\underline{\tilde{r}}-u+w) \\
& +\delta(\tilde{u}-\tilde{w}-u+w) \delta(\tilde{q}-\underline{\tilde{r}}-q+\underline{r})) .
\end{aligned}
$$

Let us consider the contribution to (B.28) of the first term in the bracket in (B.29):

$$
\begin{aligned}
\langle\Omega, & \left.B_{\tilde{n}}\left(G_{2, \tilde{n}}\right)\left(\check{H}_{\mathrm{I}, \sigma}^{\mathrm{c}}\right)^{*} B_{\tilde{m}}\left(G_{1, \tilde{m}}\right) B_{n}^{*}\left(G_{1, n}\right) \check{H}_{\mathrm{I}, \sigma}^{\mathrm{c}} B_{m}^{*}\left(G_{2, m}\right) \Omega\right\rangle_{1} \\
:= & \int d^{3} \tilde{u} d^{3} \tilde{w} d^{3} u d^{3} w \int d^{3} \tilde{q} d^{3} \tilde{p} d^{3} q d^{3} p \int d^{3 \tilde{n}} \tilde{r} d^{3 \tilde{m}} \tilde{k} d^{3 n} r d^{3 m} k \\
& \times \check{v}_{\bar{\alpha}}^{\sigma}(\tilde{w}) \check{v}_{\bar{\alpha}}^{\sigma}(w) \bar{G}_{2, \tilde{n}}(\tilde{q} ; \tilde{r}) \bar{G}_{1, \tilde{m}}(\tilde{p} ; \tilde{k}) G_{1, n}(q ; r) G_{2, m}(p ; k) \\
& \times \delta(\tilde{p}-\tilde{k}-\tilde{u}) \delta(p-\underline{k}-u) \delta(\tilde{u}-\tilde{w}-q+\underline{r}) \delta(\tilde{q}-\underline{\tilde{r}}-u+w) \\
& \times\left\langle\Omega, a(\tilde{r})^{\tilde{n}} a(\tilde{w}) a(\tilde{k})^{\tilde{m}} a^{*}(r)^{n} a^{*}(w) a^{*}(k)^{m} \Omega\right\rangle \\
= & \int d^{3} \tilde{q} d^{3} \tilde{p} d^{3} q d^{3} p \int d^{3 \tilde{n}} \tilde{r} d^{3 \tilde{m}} \tilde{k} d^{3 n} r d^{3 m} k \\
& \times \check{v}_{\bar{\alpha}}^{\sigma}\left(\tilde{w}_{*}\right) \tilde{v}_{\bar{\alpha}}^{\sigma}\left(w_{*}\right) \bar{G}_{2, \tilde{n}}(\tilde{q} ; \tilde{r}) \bar{G}_{1, \tilde{m}}(\tilde{p} ; \tilde{k}) G_{1, n}(q ; r) G_{2, m}(p ; k) \\
& \times\left\langle\Omega, a(\tilde{r})^{\tilde{n}} a(\tilde{k})^{\tilde{m}} a\left(\tilde{w} \tilde{w}_{*}\right) a^{*}\left(w_{*}\right) a^{*}(r)^{n} a^{*}(k)^{m} \Omega\right\rangle,
\end{aligned}
$$

where in the last step we integrated over $u, w, \tilde{u}, \tilde{w}$ and set $w_{*}:=p-\underline{k}-\tilde{q}+\underline{\tilde{r}}, \tilde{w}_{*}:=$ $\tilde{p}-\underline{\tilde{k}}-q+\underline{r}$. Now we consider the expectation value of the photon creation operators:

$$
\begin{aligned}
\left\langle\Omega, a(\tilde{r})^{\tilde{n}} a(\tilde{k})^{\tilde{m}} a\left(\tilde{w}_{*}\right) a^{*}\left(w_{*}\right) a^{*}(r)^{n} a^{*}(k)^{m} \Omega\right\rangle \\
\quad=\delta\left(w_{*}-\tilde{w}_{*}\right)\left\langle\Omega, a(\tilde{r})^{\tilde{n}} a(\tilde{k})^{\tilde{m}} a^{*}(r)^{n} a^{*}(k)^{m} \Omega\right\rangle,
\end{aligned}
$$

for $r, k, w, \tilde{r}, \tilde{k}, \tilde{w}$ in the supports of the respective functions. (Here we made use of the fact that $\left|\tilde{w}_{*}\right| \leq \sigma$, whereas $\left|r_{i}\right| \geq \sigma,\left|k_{j}\right| \geq \sigma$ ). Let us now substitute the r.h.s. of (B.31) to (B.30). Making use of Lemma B.2, we obtain

$$
\begin{aligned}
\langle\Omega, & \left.B_{\tilde{n}}\left(G_{2, \tilde{n}}\right)\left(\check{H}_{\mathrm{I}, \sigma}^{\mathrm{c}}\right)^{*} B_{\tilde{m}}\left(G_{1, \tilde{m}}\right) B_{n}^{*}\left(G_{1, n}\right) \check{H}_{\mathrm{I}, \sigma}^{\mathrm{c}} B_{m}^{*}\left(G_{2, m}\right) \Omega\right\rangle_{1} \\
= & \sum_{\rho \in S_{m+n}} \int d^{3} \tilde{q} d^{3} \tilde{p} d^{3} q d^{3} p \int d^{3 \tilde{n}} \tilde{r} d^{3 \tilde{m}} \tilde{k} d^{3 n} r d^{3 m} k \\
& \times \check{v}_{\bar{\alpha}}^{\sigma}(\tilde{p}-\tilde{\tilde{k}}-q+\underline{r}) \check{v}_{\bar{\alpha}}^{\sigma}(p-\underline{k}-\tilde{q}+\underline{\tilde{r}}) \bar{G}_{2, \tilde{n}}(\tilde{q} ; \tilde{r}) \bar{G}_{1, \tilde{m}}(\tilde{p} ; \tilde{k}) G_{1, n}(q ; r) G_{2, m}(p ; k) \\
& \times \delta(p+q-\tilde{p}-\tilde{q}) \delta(\hat{\tilde{r}}-\hat{r}) \delta(\tilde{\tilde{r}}-\check{k}) \delta(\hat{\tilde{k}}-\hat{k}) \delta(\tilde{\tilde{k}}-\check{r}) .
\end{aligned}
$$

By integrating over $\tilde{q}, \tilde{r}, \tilde{k}$, we obtain 


$$
\begin{aligned}
\langle\Omega, & \left.B_{\tilde{n}}\left(G_{2, \tilde{n}}\right)\left(\check{H}_{\mathrm{I}, \sigma}^{\mathrm{c}}\right)^{*} B_{\tilde{m}}\left(G_{1, \tilde{m}}\right) B_{n}^{*}\left(G_{1, n}\right) \check{H}_{\mathrm{I}, \sigma}^{\mathrm{c}} B_{m}^{*}\left(G_{2, m}\right) \Omega\right\rangle_{1} \\
= & \sum_{\rho \in S_{m+n}} \int d^{3} \tilde{p} d^{3} q d^{3} p \int d^{3 n} r d^{3 m} k \check{v}_{\bar{\alpha}}^{\sigma}(\tilde{p}-q-\underline{\hat{k}}+\underline{\hat{r}})^{2} \\
& \times \bar{G}_{2, \tilde{n}}(p+q-\tilde{p} ; \hat{r}, \check{k}) \bar{G}_{1, \tilde{m}}(\tilde{p} ; \hat{k}, \check{r}) G_{1, n}(q ; r) G_{2, m}(p ; k) \\
= & \sum_{\rho \in S_{m+n}} \int d^{3} \tilde{p} d^{3} q d^{3} p \int d^{3 n} r d^{3 m} k \check{v}_{\tilde{\alpha}}^{\sigma}(\tilde{p})^{2} \\
& \times \bar{G}_{2, \tilde{n}}(p-\tilde{p}-\underline{\hat{k}}+\underline{\hat{r}} ; \hat{r}, \check{k}) \bar{G}_{1, \tilde{m}}(\tilde{p}+q+\underline{\hat{k}}-\underline{\hat{r}} ; \hat{k}, \check{r}) G_{1, n}(q ; r) G_{2, m}(p ; k),
\end{aligned}
$$

where in the last step we made a change of variables $\tilde{p} \rightarrow \tilde{p}+q+\underline{\hat{k}}-\underline{\hat{r}}$. This gives the first term on the r.h.s. of (B.27).

Let us now consider the contribution of the second term in the bracket on the r.h.s. of formula (B.29):

$$
\begin{aligned}
& \left\langle\Omega, B_{\tilde{n}}\left(G_{2, \tilde{n}}\right)\left(\check{H}_{\mathrm{I}, \sigma}^{\mathrm{c}}\right)^{*} B_{\tilde{m}}\left(G_{1, \tilde{m}}\right) B_{n}^{*}\left(G_{1, n}\right) \check{H}_{\mathrm{I}, \sigma}^{\mathrm{c}} B_{m}^{*}\left(G_{2, m}\right) \Omega\right\rangle_{2} \\
& :=\int d^{3} w \int d^{3} \tilde{p} d^{3} q d^{3} p \int d^{3 \tilde{n}} \tilde{r} d^{3 \tilde{m}} \tilde{k} d^{3 n} r d^{3 m} k \check{v}_{\bar{\alpha}}^{\sigma}(\tilde{p}-\underline{\tilde{k}}-p+\underline{k}+w) \check{v}_{\bar{\alpha}}^{\sigma}(w) \\
& \times \bar{G}_{2, \tilde{n}}(q+\underline{\tilde{r}}-\underline{r} ; \tilde{r}) \bar{G}_{1, \tilde{m}}(\tilde{p} ; \tilde{k}) G_{1, n}(q ; r) G_{2, m}(p ; k) \\
& \times\left\langle\Omega, a(\tilde{r})^{\tilde{n}} a(\tilde{p}-\underline{\tilde{k}}-p+\underline{k}+w) a(\tilde{k})^{\tilde{m}} a^{*}(r)^{n} a^{*}(w) a^{*}(k)^{m} \Omega\right\rangle \\
& =\int d^{3} w \int d^{3} \tilde{p} d^{3} q d^{3} p \int d^{3 \tilde{n}} \tilde{r} d^{3 \tilde{m}} \tilde{k} d^{3 n} r d^{3 m} k \check{v}_{\bar{\alpha}}^{\sigma}(\tilde{p}-\underline{\tilde{k}}-p+\underline{k}+w) \check{v}_{\bar{\alpha}}^{\sigma}(w) \\
& \times \bar{G}_{2, \tilde{n}}(q+\underline{\tilde{r}}-\underline{r} ; \tilde{r}) \bar{G}_{1, \tilde{m}}(\tilde{p} ; \tilde{k}) G_{1, n}(q ; r) G_{2, m}(p ; k) \delta(\tilde{p}-\underline{\tilde{k}}-p+\underline{k}) \\
& \times\left\langle\Omega, a(\tilde{r})^{\tilde{n}} a(\tilde{k})^{\tilde{m}} a^{*}(r)^{n} a^{*}(k)^{m} \Omega\right\rangle \\
& =\left\|\check{v}_{\bar{\alpha}}^{\sigma}\right\|_{2}^{2} \sum_{\rho \in S_{m+n}} \int d^{3} q d^{3} p \int d^{3 n} r d^{3 m} k G_{1, n}(q ; r) G_{2, m}(p ; k) \\
& \times \bar{G}_{2, \tilde{n}}(q+\underline{\check{k}}-\underline{\check{r}} ; \hat{r}, \check{k}) \bar{G}_{1, \tilde{m}}(p-\underline{\check{k}}+\underline{\check{r}} ; \hat{k}, \check{r}),
\end{aligned}
$$

where in the first step we integrated over $\tilde{u}, \tilde{w}, u, \tilde{q}$ and in the last step we made use again of Lemma B.2. This gives the second term on the r.h.s. of (B.27) and concludes the proof.

\section{References}

1. Abdesselam, A., Hasler, D.: Analyticity of the ground state energy for massless Nelson models. Commun. Math. Phys. 310, 511-536 (2012)

2. Albeverio, S.: Scattering theory in a model of quantum fields. I. J. Math. Phys. 14, 1800-1816 (1973)

3. Albeverio, S.: Scattering theory in a model of quantum fields II. Helv. Phys. Acta 45, 303-321 (1972)

4. Bach, V., Chen, T., Fröhlich, J., Sigal, I.M.: The renormalized electron mass in non-relativistic quantum electrodynamics. J. Funct. Anal. 243, 426-535 (2007)

5. Chen, T., Fröhlich, J., Pizzo, A.: Infraparticle scattering states in non-relativistic QED: I. The BlochNordsieck paradigm. Commun. Math. Phys. 294, 761-825 (2010)

6. Chen, T., Fröhlich, J., Pizzo, A.: Infraparticle scattering states in non-relativistic QED: II. Mass shell properties. J. Math. Phys. 50, 012103-012134 (2009) 
7. De Roeck, W., Kupiainen, A.: Approach to ground state and time-independent photon bound for massless spin-boson models. Ann. Henri Poincaré 14(2), 253-311 (2013)

8. De Roeck, W., Griesemer, M., Kupiainen, A.: Asymptotic completeness for the massless spin-boson model. Preprint: arXiv:1301.2357

9. Dereziński, J., Gérard, C.: Asymptotic completeness in quantum field theory. Massive Pauli-Fierz Hamiltonians. Rev. Math. Phys. 11, 383-450 (1999)

10. Dereziński, J., Gérard, C.: Scattering theory of infrared divergent Pauli-Fierz Hamiltonians. Ann. Henri Poincaré 5, 523-577 (2004)

11. Dybalski, W.: Haag-Ruelle scattering theory in presence of massless particles. Lett. Math. Phys. 72, 27-38 (2005)

12. Dybalski, W., Pizzo, A.: Coulomb scattering in the massless Nelson model II. Regularity of ground states. Preprint: arXiv:1302.5012

13. Faupin, J., Sigal, I.M.: On Rayleigh scattering in non-relativistic quantum electrodynamics. Preprint: arXiv: 1211.0268

14. Fröhlich, J.: Unpublished notes

15. Fröhlich, J.: On the infrared problem in a model of scalar electrons and massless, scalar bosons. Ann. Inst. Henri Poincaré, a Phys. Théor. 19, 1-103 (1973)

16. Fröhlich, J.: Existence of dressed one electron states in a class of persistent models. Fortschr. Phys. 22, 158-198 (1974)

17. Fröhlich, J., Pizzo, A.: Renormalized electron mass in non-relativistic QED. Commun. Math. Phys. 294, 439-470 (2010)

18. Fröhlich, J., Griesemer, M., Schlein, B.: Asymptotic electromagnetic fields in models of quantummechanical matter interacting with the quantized radiation field. Adv. Math. 164, 349-398 (2001)

19. Fröhlich, J., Griesemer, M., Schlein, B.: Asymptotic completeness for Rayleigh scattering. Ann. Henri Poincaré 3, 107-170 (2002)

20. Fröhlich, J., Griesemer, M., Schlein, B.: Asymptotic completeness for Compton scattering. Commun. Math. Phys. 252, 415-476 (2004)

21. Haag, R.: Quantum field theories with composite particles and asymptotic conditions. Phys. Rev. 112, 669-673 (1958)

22. Hübner, M., Spohn, H.: Radiative decay: nonperturbative approaches. Rev. Math. Phys. 7, 363-387 (1995)

23. Könenberg, M., Matte, O.: The mass-shell in the semi-relativistic Pauli-Fierz model. Preprint: arXiv: 1204.5123

24. Pizzo, A.: One-particle (improper) states in Nelson's massless model. Ann. Henri Poincaré 4, 439-486 (2003)

25. Pizzo, A.: Scattering of an infraparticle: the one particle sector in Nelson's massless models. Ann. Henri Poincaré 4, 553-606 (2005)

26. Reed, M., Simon, B.: Methods of Modern Mathematical Physics II. Fourier Analysis, Self-adjointness. Academic Press, San Diego (1980)

27. Ruelle, D.: On the asymptotic condition in quantum field theory. Helv. Phys. Acta 35, 147-163 (1962)

28. Spohn, H.: Asymptotic completeness for Rayleigh scattering. J. Math. Phys. 38, 2281-2296 (1997)

29. Spohn, H.: Dynamics of Charged Particles and Their Radiation Field. Cambridge University Press, Cambridge (2004) 